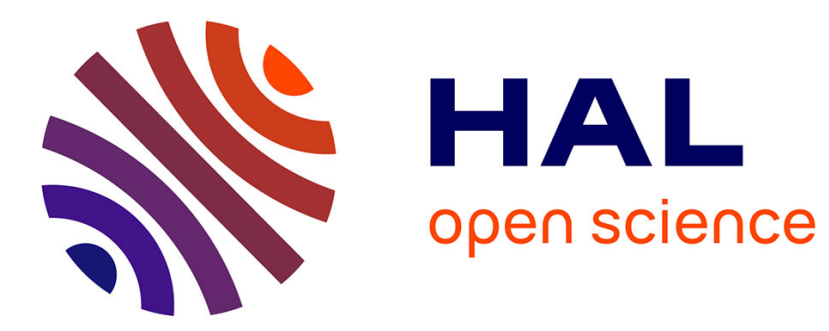

\title{
Homogenization of Stratified Elastic Composites with High Contrast
}

\author{
Michel Bellieud
}

\section{To cite this version:}

Michel Bellieud. Homogenization of Stratified Elastic Composites with High Contrast . SIAM Journal on Mathematical Analysis, 2017, 49 (4), pp.2615 - 2665. 10.1137/15M1012050 . hal-01769898

\section{HAL Id: hal-01769898 \\ https://hal.umontpellier.fr/hal-01769898}

Submitted on 18 Apr 2018

HAL is a multi-disciplinary open access archive for the deposit and dissemination of scientific research documents, whether they are published or not. The documents may come from teaching and research institutions in France or abroad, or from public or private research centers.
L'archive ouverte pluridisciplinaire HAL, est destinée au dépôt et à la diffusion de documents scientifiques de niveau recherche, publiés ou non, émanant des établissements d'enseignement et de recherche français ou étrangers, des laboratoires publics ou privés. 


\title{
HOMOGENIZATION OF STRATIFIED ELASTIC COMPOSITES WITH HIGH CONTRAST
}

\author{
MICHEL BELLIEUD *
}

\begin{abstract}
We determine the asymptotic behavior of the solutions to the linear elastodynamic equations in a stratified media comprising an alternation of possibly very stiff layers with much softer ones, when the thickness of the layers tends to zero. The limit equations may depend on higher order terms, characterizing bending effects. A part of this work is set in the context of non-periodic homogenization and an extension to stochastic homogenization is presented.
\end{abstract}

Key words. homogenization, elasticity, non-local effects

AMS subject classifications. 35B27, 35B40, 35R60, 74B05, 74Q10

1. Introduction. In this paper we analyze the asymptotic behavior of the solution to the linear elastodynamic equations in a composite material wherein, at a microscopic scale, possibly very "stiff" layers alternate with a much "softer" medium. Stratified composite media have been intensively investigated over the last decades, especially in the context of diffusion equations [18, 27, 29, 30, 31, 32, 39, 52, 54]. As regards linear elasticity, layered elastic composites have been studied in [26, 28, 33, 38] under assumptions of uniform boundedness and uniform definite positiveness of the elasticity tensor guaranteeing that the effective equation is a standart linear elasticity equation. When these assumptions break down, as for instance in the so-called "high contrast case", the limit equilibrium equation may be of a quite different type: it may correspond, in theory, to the Euler equation associated to the minimization of any lower semi-continuous quadratic form on $L^{2}$ vanishing on rigid motions [20]. In particular, it may be non-local and depend on higher order derivatives of the displacement. Elastic media with high contrast have been studied under various geometrical assumptions. Composites with stiff grain-like inclusions have been investigated in $[7,8,45]$, stiff fibered structures in $[8,12,13,46,50]$, and stiff media with holes filled with a soft material in [22, 24, 47]. Our aim is to complement this body of work in the context of stratified media. Our approach is based on the two-scale convergence method $[3,5,19,23,40,41]$, which yields the convergence to an effective solution. It also yields a first order corrector result in $L^{2}$ (see Remark 3.14), but not the rigorous error estimates of higher order with respect to small parameters provided by the asymptotic expansions method [1, 2, 6, 15, 16, 21, 43, 44, 45, 48, 49].

For a given bounded smooth open subset $\Omega$ of $\mathbb{R}^{3}$, we consider a linear elastodynamic problem like (3.5). We assume that the Lamé coefficients take possibly large values in a subset $B_{\varepsilon}$ of $\Omega$ and much smaller values elsewhere. The set $B_{\varepsilon}$ consists of a non-periodic distribution of parallel disjoint homothetic layers of thickness $r_{\varepsilon}$, whose median planes are orthogonal to $\boldsymbol{e}_{3}$ and separated by a minimal distance $\varepsilon$, where $\varepsilon, r_{\varepsilon}$ are positive reals converging to zero (see fig. 3.1). The effective volume fraction of the stiff phase $B_{\varepsilon}$ is characterized by the parameter $\vartheta$ defined by (3.10). Both cases $\vartheta=0$ and $0<\vartheta<1$ are investigated. The order of magnitude of the Lamé coefficients in the stiff phase is determined by the parameters $k$ and $\kappa$ defined by (3.8).

When the elasticity coefficients in the soft phase are of order 1 and the effective

* Université Montpellier 2, Case courier 048, Place Eugène Bataillon, 34095 Montpellier Cedex 5, France. (michel.bellieud@univ-monpt2.fr). 
volume fraction of the stiff phase vanishes the limit behavior of the composite is governed, if $0<k<+\infty$, by the equation

$$
\left(\rho+n \bar{\rho}_{1}\right) \frac{\partial^{2} \boldsymbol{u}}{\partial t^{2}}-\operatorname{div} \boldsymbol{\sigma}(\boldsymbol{u})-n k \operatorname{div} \boldsymbol{\sigma}_{x^{\prime}}\left(\boldsymbol{u}^{\prime}\right)=\left(\rho+n \bar{\rho}_{1}\right) \boldsymbol{f} \quad \text { in } \Omega \times(0, T),
$$

where $\rho$ denotes the mass density in the softer phase, and $\boldsymbol{u}^{\prime}, \boldsymbol{\sigma}_{x^{\prime}}, \boldsymbol{\sigma}$ and $\bar{\rho}_{1}$ are defined, respectively, by (2.1), (3.13), and (3.7). The function $n$ characterizes the rescalled effective number of sections of stiff layers per unit length in the $\boldsymbol{e}_{3}$ direction and is obtained as the weak* limit in $L^{\infty}(\Omega)$ of the sequence $\left(n_{\varepsilon}\right)$ defined by (3.14). When the order of magnitude of the elasticity coefficients in the stiff layers is larger, that is when $k=+\infty$, the functions $u_{1}$ and $u_{2}$ vanish on the set $\{n>0\}$ and the behavior of $u_{3}$ is governed by the equation (3.19), (3.20) or (3.21), depending on the order of magnitude of $\kappa$. In the case $0<\kappa<+\infty$, this equation involves the $4^{t h}$ partial derivatives of $u_{3}$ with respect to $x_{1}, x_{2}$ :

$$
\begin{aligned}
\left(\rho+n \bar{\rho}_{1}\right) \frac{\partial^{2} u_{3}}{\partial t^{2}}-(\operatorname{div} \boldsymbol{\sigma}(\boldsymbol{u}))_{3}+n \frac{\kappa}{3} \frac{l+1}{l+2} \sum_{\alpha, \beta=1}^{2} \frac{\partial^{4} u_{3}}{\partial x_{\alpha}^{2} \partial x_{\beta}^{2}} & \\
& =\left(\rho+n \bar{\rho}_{1}\right) f_{3} \quad \text { in } \Omega \times(0, T),
\end{aligned}
$$

revealing bending effects. The effective behavior on the set $\{n=0\}$ is that of a homogeneous material without stiff layers. In Theorem 3.5, we extend these results to the stochastic case. The set $B_{\varepsilon}(\omega)$ then depends on a random element $\omega$ of some sample space $\mathfrak{O} \subset 2^{\mathbb{R}}$ equiped with a probability $P$ satisfying (3.23). The limit problem as $\varepsilon \rightarrow 0$ is deduced from the above equations, $P$-almost surely, by substituting for $n$ the conditional expectation $E_{P}^{\mathcal{F}} n_{0}(\omega)$ with respect to $P$ given the $\sigma$-algebra $\mathcal{F}$ of the periodic sets, of the random variable $n_{0}$ defined by (3.24).

If the order of magnitude of the elasticity coefficients in the soft interlayers is strictly smaller than 1 and strictly larger than $\varepsilon^{2}$, the effective equations are deduced from (1.1), (1.2), formally, by removing the term $\operatorname{div} \boldsymbol{\sigma}(\boldsymbol{u})$ (see Theorem 3.7).

When the elastic moduli in the soft phase are of order $\varepsilon^{2}$, the effective behavior of the composite turns sensitive to the slightest geometrical perturbation (see Remark 3.20). The effective equation can not be expressed simply in terms of the function $n$ as in the other cases. This characteristic renders the study of non-periodic homogenization a very difficult task: we only treat the case of an $\varepsilon$-periodic distribution of stiff layers. The homogenized problem then takes the form of a system of equations coupling some field $\boldsymbol{v}$, characterizing the effective displacement in the stiff layers, with the two-scale limit $\boldsymbol{u}_{0}: \Omega \times(0, T) \times\left(-\frac{1}{2}, \frac{1}{2}\right)^{3} \rightarrow \mathbb{R}^{3}$ of the solution $\left(\boldsymbol{u}_{\varepsilon}\right)$ to (3.5) (see $[3,41])$. This field $\boldsymbol{v}$ is obtained as the limit of the sequence $\left(\boldsymbol{u}_{\varepsilon} m_{\varepsilon}\right)$, where $m_{\varepsilon}$ is the measure supported by the stiff layers defined by (3.39). If $0<k<+\infty$, the effective behavior of the displacement in the stiff medium is governed by the equation

$$
\bar{\rho}_{1} \frac{\partial^{2} \boldsymbol{v}}{\partial t^{2}}-k \operatorname{div} \boldsymbol{\sigma}_{x^{\prime}}\left(\boldsymbol{v}^{\prime}\right)=\bar{\rho}_{1} \boldsymbol{f}+\boldsymbol{g}\left(\boldsymbol{u}_{0}\right) \quad \text { in } \Omega \times(0, T),
$$

associated with the boundary and initial conditions given in (3.45). This equation displays stretching vibrations with regard to the transversal components $v_{1}, v_{2}$ of $\boldsymbol{v}$. It is coupled with the soft phase through the field $\boldsymbol{g}\left(\boldsymbol{u}_{0}\right)$ which represents the sum of 
the surface forces applied on each stiff layer by the adjacent soft medium. This field is defined by (3.42), in terms of the restriction of $\boldsymbol{u}_{0}$ to $\Omega \times(0, T) \times Y \backslash A$, which characterizes the effective displacement in the soft interstitial layers. The letters $Y$ and $A$ denote, respectively, the unit cell and the rescaled stiff layer (see (3.36), (3.37)). The effective displacement in the soft phase is governed by the equation

$$
\rho \frac{\partial^{2} \boldsymbol{u}_{0}}{\partial t^{2}}-\operatorname{div}_{y}\left(\boldsymbol{\sigma}_{0 y}\left(\boldsymbol{u}_{0}\right)\right)=\rho \boldsymbol{f} \quad \text { in } \Omega \times(0, T) \times Y \backslash A,
$$

where $\boldsymbol{\sigma}_{0 y}$ is defined by (3.42). This equation is coupled with the variable $\boldsymbol{v}$ by the relation (3.41) on $\Omega \times(0, T) \times A$. The weak limit of $\left(\boldsymbol{u}_{\varepsilon}\right)$ in $L^{2}(\Omega \times(0, T))$ is given by $\boldsymbol{u}(x, t)=\int_{Y} \boldsymbol{u}_{0}(x, t, y) d y$.

When the order of magnitude of the elasticity coefficients in the stiff layers is larger, the functions $v_{1}$ and $v_{2}$ vanish and the effective displacement in the stiff phase is governed by the equation of $v_{3}$ given by (3.46), (3.47) or (3.48), depending on the order of magnitude of $\kappa$. In the case $0<\kappa<+\infty$, this equation,

$$
\bar{\rho}_{1} \frac{\partial^{2} v_{3}}{\partial t^{2}}+\frac{\kappa}{3} \frac{l+1}{l+2} \sum_{\alpha, \beta=1}^{2} \frac{\partial^{4} v_{3}}{\partial x_{\alpha}^{2} \partial x_{\beta}^{2}}=\bar{\rho}_{1} f_{3}+\left(\boldsymbol{g}\left(\boldsymbol{u}_{0}\right)\right)_{3} \quad \text { in } \Omega \times(0, T),
$$

involves the $4^{\text {th }}$ partial derivatives of $v_{3}$ with respect to $x_{1}, x_{2}$, characterizing bending vibrations. Otherwise, the stiff layers display the behavior of a collection of unstretchable membranes if $(k, \kappa)=(+\infty, 0)$ and that of fixed bodies if $\kappa=\infty$.

Our results apply as well to equilibrium equations (see remarks 3.11, 3.19) and can be extended to multiphase composites (see remarks 3.10, 3.16, 3.17). Multiphase homogenized models have been studied in [8, 44, 45, 47, 48, 49].

As occurs in the case of fibers or grain-like inclusions embedded in a soft matrix (see $[8$, p.3]), the two-phase stratified composites modeled above by homogenization prove to be, in general, unsufficiently reinforced to "resist" to body forces. Accordingly, some of the components of the effective displacement may exhibit a quadratic behavior with respect to time, describing a motion of collapse. The whole displace-

ment $\boldsymbol{u}$ does so, for instance, on the set $\widehat{\{n=0\}}$ in (3.28)-(3.30). If $\kappa=0$, a similar behavior is shown by $u_{3}$ in (3.28), (3.29), and by $v_{3}$ in (3.45), (3.46), if $\boldsymbol{f}$ is not parallel to the layers. In these cases, the sequence of the solutions to the associated equilibrium problems (see (3.32)) may fail to be bounded in $L^{2}$, and the effective equilibrium problem to be well-posed. Boundedness in $L^{2}$ is achieved by considering suitable multiphase media. $\mathrm{n}$ the fibered case, the choice of such media has been discussed in [8, Proposition 5.2]. We extend this discussion in remarks 3.11, 3.19, 6.3.

The paper is organised as follows: in Section 2 we specify the notations and in Section 3 we state our main results. In Section 4, we recall some classical results and introduce a non-periodic variant of the two-scale convergence for which we establish a compactness result. The effective equations are derived in Section 6 by employing apriori estimates demonstrated in Section 5, and a technical lemma proved in the appendix.

2. Notations. In this article, $\left\{\boldsymbol{e}_{1}, . ., \boldsymbol{e}_{N}\right\}$ stands for the canonical basis of $\mathbb{R}^{N}$. Points in $\mathbb{R}^{N}$ or in $\mathbb{Z}^{N}$ and real-valued functions are represented by symbols beginning by a lightface lowercase (example $x, i, \operatorname{det} \boldsymbol{A} \ldots$ ) and vectors and vector-valued functions by symbols beginning by a boldface lowercase (examples: $\left.\boldsymbol{x}, \boldsymbol{i}, \boldsymbol{u}, \boldsymbol{f}, \boldsymbol{g}, \boldsymbol{\operatorname { d i v }} \boldsymbol{\sigma}_{\varepsilon}, \ldots\right)$. 
Matrices and matrix-valued functions are represented by symbols beginning by a boldface uppercase with the following exceptions: $\boldsymbol{\nabla} \boldsymbol{u}$ (displacement gradient), $\boldsymbol{e}(\boldsymbol{u})$ (linearized strain tensor). We denote by $u_{i}$ or $(\boldsymbol{u})_{i}$ the components of a vector $\boldsymbol{u}$ and by $A_{i j}$ or $(\boldsymbol{A})_{i j}$ those of a matrix $\boldsymbol{A}$ (that is $\boldsymbol{u}=\sum_{i=1}^{N} u_{i} \boldsymbol{e}_{i}=\sum_{i=1}^{N}(\boldsymbol{u})_{i} \boldsymbol{e}_{i} ; \boldsymbol{A}=$ $\left.\sum_{i, j=1}^{N} A_{i j} \boldsymbol{e}_{i} \otimes \boldsymbol{e}_{j}=\sum_{i, j=1}^{N}(\boldsymbol{A})_{i j} \boldsymbol{e}_{i} \otimes \boldsymbol{e}_{j}\right)$. We do not employ the usual repeated index convention for summation. We denote by $\boldsymbol{A}: \boldsymbol{B}=\sum_{i, j=1}^{N} A_{i j} B_{i j}$ the inner product of two matrices, by $\varepsilon_{i j k}$ the three-dimensional alternator, by $\boldsymbol{u} \wedge \boldsymbol{v}=\sum_{i, j, k=1}^{3} \varepsilon_{i j k} u_{j} v_{k} \boldsymbol{e}_{i}$ the exterior product in $\mathbb{R}^{3}$, by $\mathbb{S}^{M}(M \in \mathbb{N})$ the set of all real symmetric matrices of order $M$, by $\boldsymbol{I}_{M}$ the $M \times M$ identity matrix. The symbol $\sharp D$ denotes the cardinality of a finite set $D$. The letter $C$ stands for different constants independent of $\varepsilon$ whose precise values may vary. For any weakly differentiable vector field $\psi: \Omega \subset \mathbb{R}^{3} \rightarrow \mathbb{R}^{3}$, we set

$$
\begin{gathered}
\boldsymbol{\psi}^{\prime}:=\psi_{1} \boldsymbol{e}_{1}+\psi_{2} \boldsymbol{e}_{2} ; \quad \boldsymbol{e}_{x^{\prime}}(\boldsymbol{\psi}):=\sum_{\alpha, \beta=1}^{2} \frac{1}{2}\left(\frac{\partial \psi_{\alpha}}{\partial x_{\beta}}+\frac{\partial \psi_{\beta}}{\partial x_{\alpha}}\right) \boldsymbol{e}_{\alpha} \otimes \boldsymbol{e}_{\beta}\left(=\boldsymbol{e}_{x^{\prime}}\left(\boldsymbol{\psi}^{\prime}\right)\right) \\
\boldsymbol{\sigma}_{x^{\prime}}(\boldsymbol{\psi}):=\left(\begin{array}{ccc}
2 \frac{\partial \psi_{1}}{\partial x_{1}}+\frac{2 l}{l+2}\left(\frac{\partial \psi_{1}}{\partial x_{1}}+\frac{\partial \psi_{2}}{\partial x_{2}}\right) & \frac{\partial \psi_{1}}{\partial x_{2}}+\frac{\partial \psi_{2}}{\partial x_{1}} & 0 \\
\frac{\partial \psi_{1}}{\partial x_{2}}+\frac{\partial \psi_{2}}{\partial x_{1}} & 2 \frac{\partial \psi_{2}}{\partial x_{2}}+\frac{2 l}{l+2}\left(\frac{\partial \psi_{1}}{\partial x_{1}}+\frac{\partial \psi_{2}}{\partial x_{2}}\right) & 0 \\
0 & 0 & 0
\end{array}\right)
\end{gathered}
$$

where the non-negative parameter $l$ is defined in (3.6). We reproduce and modify here some notations from [8]: we denote by $C_{\sharp}^{\infty}(Y)$ (resp. $\left.C_{\sharp}(Y)\right)$ the set of $Y$-periodic functions from $C^{\infty}\left(\mathbb{R}^{3}\right)$ (resp. $C\left(\mathbb{R}^{3}\right)$ ), by $C_{\sharp}^{\infty}(Y \backslash B)$ the set of the restrictions of the elements of $C_{\sharp}^{\infty}(Y)$ to $Y \backslash B$, by $H_{\sharp}^{1}(Y)$ (resp. $H_{\sharp}^{1}(Y \backslash B)$ ) the completion of $C_{\sharp}^{\infty}(Y)$ (resp. $\left.C_{\sharp}^{\infty}(Y \backslash B)\right)$ with respect to the norm $w \rightarrow\left(\int_{Y}\left(|w|^{2}+|\nabla w|^{2}\right) d y\right)^{\frac{1}{2}}$ ( resp. $w \rightarrow\left(\int_{Y \backslash B}\left(|w|^{2}+|\nabla w|^{2}\right) d y\right)^{\frac{1}{2}}$ ). For any subset $Q$ of the unit cell $Y$, the symbol $Q_{\sharp}$ stands for the periodization on all $\mathbb{R}^{3}$ of $Q$, that is

$$
Q_{\sharp}:=\bigcup_{z \in \mathbb{Z}^{3}} z+Q .
$$

3. Setting of the problem and results. We consider a cylindrical domain $\Omega:=\Omega^{\prime} \times(0, L)$, where $\Omega^{\prime}$ is a bounded smooth domain of $\mathbb{R}^{2}$. Given a small positive real $\varepsilon$, the non-periodic distribution $B_{\varepsilon}$ of disjoint homothetical stiff layers $B_{\varepsilon}^{j}$ will be described in terms of a finite subset $\omega_{\varepsilon}$ of $\mathbb{R}$

$$
\omega_{\varepsilon}:=\left\{\omega_{\varepsilon}^{j}, j \in J_{\varepsilon}\right\}, \quad J_{\varepsilon}:=\left\{1, . ., \sharp \omega_{\varepsilon}\right\},
$$

satisfying

$$
\omega_{\varepsilon} \subset(0, L), \quad \min _{j, j^{\prime} \in J_{\varepsilon}, j \neq j^{\prime}}\left|\omega_{\varepsilon}^{j}-\omega_{\varepsilon}^{j^{\prime}}\right|=\varepsilon, \quad \operatorname{dist}\left(\omega_{\varepsilon},\{0, L\}\right)>\frac{\varepsilon}{2},
$$

and of a small parameters $r_{\varepsilon}$ verifying

$$
\varepsilon>r_{\varepsilon}(1+\delta) \quad \text { for some } \delta>0,
$$


by setting (see Fig. 3.1)

$$
B_{\varepsilon}:=\bigcup_{j \in J_{\varepsilon}} B_{\varepsilon}^{j} ; \quad B_{\varepsilon}^{j}:=\Omega^{\prime} \times\left(\omega_{\varepsilon}^{j}+r_{\varepsilon} I\right) ; \quad I:=\left(-\frac{1}{2}, \frac{1}{2}\right) .
$$

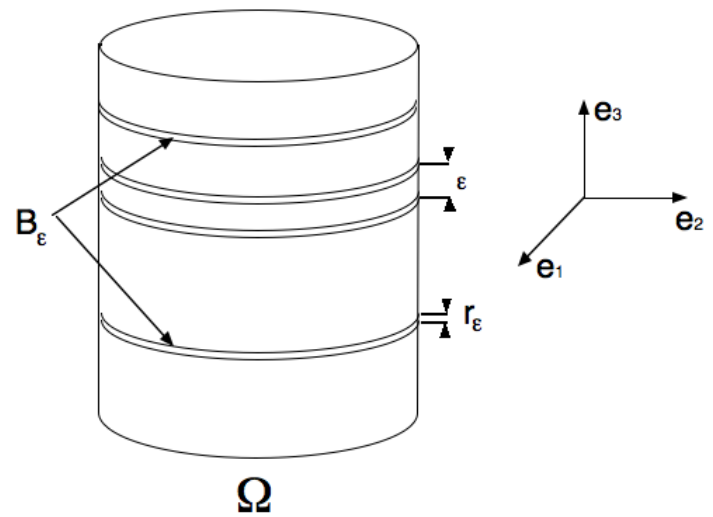

FIG. 3.1.

As in [8], we consider the vibration problem

$$
\left(\mathcal{P}_{\varepsilon}\right):\left\{\begin{array}{l}
\rho_{\varepsilon} \frac{\partial^{2} \boldsymbol{u}_{\varepsilon}}{\partial t^{2}}-\operatorname{div}\left(\boldsymbol{\sigma}_{\varepsilon}\left(\boldsymbol{u}_{\varepsilon}\right)\right)=\rho_{\varepsilon} \boldsymbol{f} \quad \text { in } \Omega \times(0, T), \\
\boldsymbol{\sigma}_{\varepsilon}\left(\boldsymbol{u}_{\varepsilon}\right)=\lambda_{\varepsilon} \operatorname{tr}\left(\boldsymbol{e}\left(\boldsymbol{u}_{\varepsilon}\right)\right) \boldsymbol{I}+2 \mu_{\varepsilon} \boldsymbol{e}\left(\boldsymbol{u}_{\varepsilon}\right), \quad \boldsymbol{e}\left(\boldsymbol{u}_{\varepsilon}\right)=\frac{1}{2}\left(\nabla \boldsymbol{u}_{\varepsilon}+\nabla^{T} \boldsymbol{u}_{\varepsilon}\right), \\
\boldsymbol{u}_{\varepsilon} \in C\left([0, T] ; H_{0}^{1}\left(\Omega, \mathbb{R}^{3}\right)\right) \cap C^{1}\left([0, T] ; L^{2}\left(\Omega, \mathbb{R}^{3}\right)\right), \\
\boldsymbol{u}_{\varepsilon}(0)=\boldsymbol{a}_{0}, \quad \frac{\partial \boldsymbol{u}_{\varepsilon}}{\partial t}(0)=\boldsymbol{b}_{0}, \quad \boldsymbol{f} \in C\left(\overline{\Omega \times(0, T)} ; \mathbb{R}^{3}\right), \\
\left(\boldsymbol{a}_{0}, \boldsymbol{b}_{0}\right) \in\left(H_{0}^{1}\left(\Omega, \mathbb{R}^{3}\right) \times L^{2}\left(\Omega, \mathbb{R}^{3}\right)\right) \cap C\left(\bar{\Omega}, \mathbb{R}^{3}\right)^{2} .
\end{array}\right.
$$

The Lamé coefficients $\mu_{\varepsilon}, \lambda_{\varepsilon}$ and the mass density $\rho_{\varepsilon}$ are assumed to take constant values of possibly different orders of magnitude in the set of layers $B_{\varepsilon}$ and in the set of interlayers $\Omega \backslash B_{\varepsilon}$. More precisely, we suppose that

$$
\begin{aligned}
& \mu_{\varepsilon}(x)=\mu_{1 \varepsilon} \mathbb{1}_{B_{\varepsilon}}(x)+\mu_{0 \varepsilon} \mathbb{1}_{\Omega \backslash B_{\varepsilon}}(x), \quad \lambda_{\varepsilon}(x)=\lambda_{1 \varepsilon} \mathbb{1}_{B_{\varepsilon}}(x)+\lambda_{0 \varepsilon} \mathbb{1}_{\Omega \backslash B_{\varepsilon}}(x), \\
& \mu_{1 \varepsilon} \geq c>0, \quad l_{\varepsilon}:=\frac{\lambda_{1 \varepsilon}}{\mu_{1 \varepsilon}}, \quad \lim _{\varepsilon \rightarrow 0} l_{\varepsilon}=l \in[0,+\infty), \quad 0 \leq \lambda_{0 \varepsilon}<C \mu_{0 \varepsilon} \ll \mu_{\varepsilon 1},
\end{aligned}
$$

and

$$
\rho_{\varepsilon}(x)=\rho \mathbb{1}_{\Omega \backslash B_{\varepsilon}}+\frac{\varepsilon}{r_{\varepsilon}} \bar{\rho}_{1} \mathbb{1}_{B_{\varepsilon}}, \quad \rho, \bar{\rho}_{1} \in(0,+\infty) .
$$

We assume and set 


$$
k:=\lim _{\varepsilon \rightarrow 0} \frac{r_{\varepsilon}}{\varepsilon} \mu_{1 \varepsilon} \in(0,+\infty], \quad \kappa:=\lim _{\varepsilon \rightarrow 0} \frac{r_{\varepsilon}^{3}}{\varepsilon} \mu_{1 \varepsilon} \in[0,+\infty] .
$$

The weak* relative compactness in $L^{\infty}\left(0, T ; L^{2}\left(\Omega ; \mathbb{R}^{3}\right)\right)$ of the sequence of the solutions to (3.5) is ensured by the following hypothesis:

$$
\sup _{\varepsilon>0} \int_{\Omega}\left(\rho_{\varepsilon}\left|\boldsymbol{b}_{0}\right|^{2}+\boldsymbol{\sigma}_{\varepsilon}\left(\boldsymbol{a}_{0}\right): \boldsymbol{e}\left(\boldsymbol{a}_{0}\right)\right) d x+\int_{\Omega \times(0, T)} \rho_{\varepsilon}|\boldsymbol{f}|^{2} d x d t<+\infty .
$$

3.1. Case of interlayers with Lamé coefficients of order 1 . We consider the case of extremely thin layers of extremely large stiffness alternating with interlayers of elastic moduli of order 1 . The effective volume fraction of the stiff layers is characterized by:

$$
\vartheta:=\lim _{\varepsilon \rightarrow 0} \frac{r_{\varepsilon}}{\varepsilon}
$$

We assume in this subsection that

$$
\begin{aligned}
& \vartheta=0, \\
& \mu_{0 \varepsilon}=\mu>0 ; \quad \lambda_{0 \varepsilon}=\lambda \geq 0 .
\end{aligned}
$$

We introduce the operator $\sigma: H^{1}\left(\Omega ; \mathbb{R}^{3}\right) \rightarrow L^{2}\left(\Omega ; \mathbb{S}^{3}\right)$ and $n_{\varepsilon} \in L^{\infty}(\Omega)$ defined by

$$
\begin{aligned}
& \boldsymbol{\sigma}(\boldsymbol{\varphi}):=\lambda \operatorname{tr}(\boldsymbol{e}(\boldsymbol{\varphi})) \boldsymbol{I}+2 \mu \boldsymbol{e}(\boldsymbol{\varphi}) \quad \forall \boldsymbol{\varphi} \in H^{1}\left(\Omega ; \mathbb{R}^{3}\right), \\
& n_{\varepsilon}(x):=\sum_{i \in Z_{\varepsilon}} \sharp\left(\omega_{\varepsilon} \cap\left(\varepsilon i-\frac{\varepsilon}{2}, \varepsilon i+\frac{\varepsilon}{2}\right]\right) \mathbb{1}_{\left(\varepsilon i-\frac{\varepsilon}{2}, \varepsilon i+\frac{\varepsilon}{2}\right)}\left(x_{3}\right), \\
& Z_{\varepsilon}:=\left\{i \in \mathbb{Z},\left(\varepsilon i-\frac{\varepsilon}{2}, \varepsilon i+\frac{\varepsilon}{2}\right] \subset(0, L)\right\} .
\end{aligned}
$$

Assumption (3.2) implies that $\left|n_{\varepsilon}\right|_{L^{\infty}(\Omega)} \leq 1$, therefore, up to a subsequence,

$$
n_{\varepsilon} \stackrel{\star}{\rightarrow} n \text { weakly* in } L^{\infty}(\Omega) \text { for some } n \in L^{\infty}(\Omega) .
$$

The scalar $\frac{1}{\varepsilon} n_{\varepsilon}(x)$ is an approximation at $x$ of the local number of stiff layers per unit length in the $\boldsymbol{e}_{3}$ direction. For simplicity (see Remark 3.3), we assume that

$$
\{n>0\} \quad \text { is a finite union of open connected subsets of } \Omega \text { if } k=+\infty \text {. }
$$

Under these assumptions, we prove that the solution to (3.5) weakly* converges in $L^{\infty}\left(0, T ; H_{0}^{1}\left(\Omega ; \mathbb{R}^{3}\right)\right)$ to the unique solution to $\left(\mathcal{P}_{(n, k, \kappa)}^{h o m}\right)$ defined, in terms of $k, \kappa, n$ given by (3.8), (3.16), as follows: if $0<k<+\infty$, we get (see (2.1))

$$
\left(\begin{array} { l } 
{ \mathcal { P } _ { ( n , k , 0 ) } ^ { h o m } ) : } \\
{ ( 0 < k < + \infty ) }
\end{array} \left\{\begin{array}{l}
\left(\rho+n \bar{\rho}_{1}\right) \frac{\partial^{2} \boldsymbol{u}}{\partial t^{2}}-\operatorname{div} \boldsymbol{\sigma}(\boldsymbol{u}) \\
\quad-n k \operatorname{div} \boldsymbol{\sigma}_{x^{\prime}}\left(\boldsymbol{u}^{\prime}\right)=\left(\rho+n \bar{\rho}_{1}\right) \boldsymbol{f} \quad \text { in } \Omega \times(0, T), \\
\boldsymbol{u} \in C\left([0, T] ; H_{0}^{1}\left(\Omega ; \mathbb{R}^{3}\right)\right) \cap C^{1}\left([0, T] ; L^{2}\left(\Omega ; \mathbb{R}^{3}\right)\right), \\
\boldsymbol{u}(0)=\boldsymbol{a}_{0}, \frac{\partial \boldsymbol{u}}{\partial t}(0)=\boldsymbol{b}_{0} .
\end{array}\right.\right.
$$


If $k=+\infty$ and $\kappa=0$, the limit problem is deduced from (3.18), formally, by substituting $(0,0)$ for $\left(u_{1}(x), u_{2}(x)\right)$ when $n(x)>0$ :

$$
\left(\mathcal{P}_{(n,+\infty, 0)}^{\text {hom }}\right):\left\{\begin{array}{l}
\left(\rho+n \bar{\rho}_{1}\right) \frac{\partial^{2} u_{3}}{\partial t^{2}}-(\operatorname{div} \boldsymbol{\sigma}(\boldsymbol{u}))_{3}=\left(\rho+n \bar{\rho}_{1}\right) f_{3} \quad \text { in } \Omega \times(0, T) \\
\rho \frac{\partial^{2} u_{\alpha}}{\partial t^{2}}-(\operatorname{div} \boldsymbol{\sigma}(\boldsymbol{u}))_{\alpha}=\rho f_{\alpha} \quad \text { in } \widehat{\{n=0\}} \times(0, T) \\
u_{\alpha}=0 \quad \text { on } \partial \widehat{\{n=0\}} \times(0, T) \quad(\alpha \in\{1,2\}) \\
n u_{1}=n u_{2}=0, \boldsymbol{u} \in C\left([0, T] ; H_{0}^{1}\left(\Omega ; \mathbb{R}^{3}\right)\right) \cap C^{1}\left([0, T] ; L^{2}\left(\Omega ; \mathbb{R}^{3}\right)\right) \\
\boldsymbol{u}(0)=\left(a_{01} \mathbb{1}_{\{n=0\}}, a_{02} \mathbb{1}_{\{n=0\}}, a_{03}\right) \\
\frac{\partial \boldsymbol{u}}{\partial t}(0)=\left(b_{01} \mathbb{1}_{\{n=0\}}, b_{02} \mathbb{1}_{\{n=0\}}, b_{03}\right)
\end{array}\right.
$$

The case $0<\kappa<+\infty$ is characterized by the emergence of fourth order derivatives with respect to $x_{1}, x_{2}$ in the limit equations, revealing bending effects:

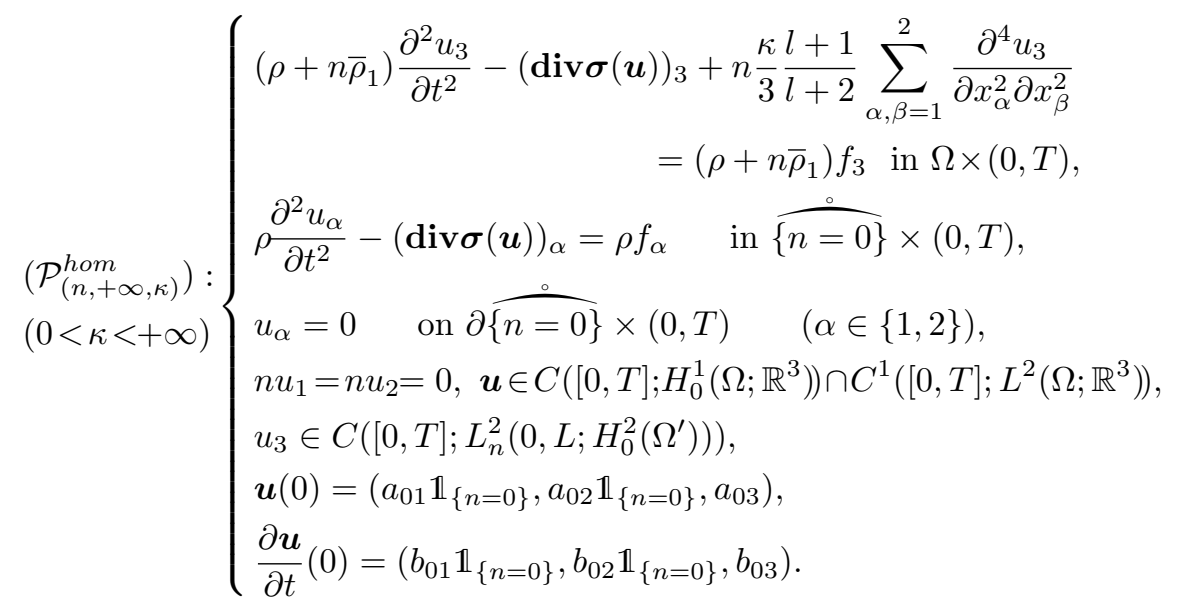

If $\kappa=+\infty$, we get:

$$
\left(\mathcal{P}_{(n,+\infty,+\infty)}^{h o m}\right):\left\{\begin{array}{l}
\rho \frac{\partial^{2} \boldsymbol{u}}{\partial t^{2}}-\operatorname{div} \boldsymbol{\sigma}(\boldsymbol{u})=\rho \boldsymbol{f} \quad \text { in } \widehat{\{n=0\}} \times(0, T) \\
\boldsymbol{u}=0 \quad \text { on } \partial \widehat{\{n=0\}} \times(0, T) \\
n \boldsymbol{u}=0, \quad \boldsymbol{u} \in C\left([0, T] ; H_{0}^{1}\left(\Omega ; \mathbb{R}^{3}\right)\right) \cap C^{1}\left([0, T] ; L^{2}\left(\Omega ; \mathbb{R}^{3}\right)\right) \\
\boldsymbol{u}(0)=\boldsymbol{a}_{0} \mathbb{1}_{\{n=0\}}, \frac{\partial \boldsymbol{u}}{\partial t}(0)=\boldsymbol{b}_{0} \mathbb{1}_{\{n=0\}}
\end{array}\right.
$$

Theorem 3.1. Assume (3.11), (3.12), (3.16), then the sequence $\left(\boldsymbol{u}_{\varepsilon}\right)$ of the solutions to (3.5) weakly* converges in $L^{\infty}\left(0, T ; H_{0}^{1}\left(\Omega ; \mathbb{R}^{3}\right)\right)$ to the unique solution of the problem $\left(\mathcal{P}_{(n, k, \kappa)}^{\text {hom }}\right)$ given by (3.18)-(3.21).

REMARK 3.2. (i) When stiff fibers [9, 13] (resp. grain-like inclusions [7]) embedded in a matrix of stiffness of order 1 are considered, the fibers (resp. the inclusions) disappear from the limit problem if $r_{\varepsilon} \ll \exp -\frac{1}{\varepsilon^{2}}$ (resp. $r_{\varepsilon} \ll \varepsilon^{3}$ ), where $r_{\varepsilon}$ denotes 
the diameter of the sections of the fibers (resp. of the inclusions). This never occurs in the stratified case, whatever the choice of $\left(r_{\varepsilon}\right)$. This is related to the fact that the harmonic capacity of a surface in $\Omega$ is always positive, whereas that of a line or a point are equal to zero.

(ii) Under (3.12), the case $\vartheta>0, k<+\infty$ has been studied in [27], [38]. In the case $\vartheta>0, k=+\infty$, we came up against technical complications (see Remark 6.1).

REMARK 3.3. If (3.17) is not satisfied, the sequence $\left(\boldsymbol{u}_{\varepsilon}\right)$ of the solutions to (3.5) weakly* converges in $L^{\infty}\left(0, T ; H_{0}^{1}\left(\Omega ; \mathbb{R}^{3}\right)\right)$ to the unique solution of the variational problem (4.23) with data deduced from (6.60), (6.61) by substituting $\widetilde{V}_{n, k, \kappa}$ and $\widetilde{a}_{n, k, \kappa}$, defined by (6.62), for $V_{n, k, \kappa}$ and $a_{n, k, \kappa}$. Assumption (3.17) ensures the equivalence between (3.19-3.21) and the last mentioned variational problem.

3.2. Stochastic case. Fix $d>0$ and set

$$
\begin{aligned}
& \mathfrak{O}:=\left\{\omega \in 2^{\mathbb{R}}, \forall\left(\omega_{1}, \omega_{2}\right) \in \omega \times \omega, \omega_{1} \neq \omega_{2} \Rightarrow\left|\omega_{1}-\omega_{2}\right| \geq d\right\}, \\
& \omega_{\varepsilon}(\omega):=\varepsilon \omega \cap(\varepsilon, L-\varepsilon) \quad \forall \omega \in \mathfrak{O},
\end{aligned}
$$

where $2^{\mathbb{R}}$ denotes the set of all subsets of $\mathbb{R}$. Let $\mathcal{B}_{\mathfrak{O}}$ be the Borel $\sigma$-algebra generated by the Hausdorff distance on $\mathfrak{O}$ (see Remark 3.6), and $P$ be a probability on $\left(\mathfrak{O}, \mathcal{B}_{\mathfrak{O}}\right.$ ) satisfying

$$
P(A+z)=P(A) \quad \forall z \in \mathbb{Z}, \forall A \in \mathcal{B}_{\mathfrak{O}}
$$

We consider the random distribution of stiff homothetical layers $B_{\varepsilon}\left(\omega_{\varepsilon}(\omega)\right)$ and the problem $\left(\mathcal{P}_{\varepsilon}(\omega)\right)$ obtained by substituting $\omega_{\varepsilon}(\omega)$ for $\omega_{\varepsilon}$ in (3.4), (3.5). In what follows, $\mathcal{F}$ represents the $\sigma$-algebra of the $Y$-periodic elements of $\mathcal{B}_{\mathfrak{O}}, E_{P}^{\mathcal{F}} X$ the conditional expectation of a random variable $X$ given $\mathcal{F}$ with respect to $P, n_{\varepsilon}(\omega)$ the element of $L^{\infty}(\Omega)$ defined by substituting $\omega_{\varepsilon}(\omega)$ for $\omega_{\varepsilon}$ in $(3.14)$, and $n_{0}: \mathfrak{O} \rightarrow \mathbb{N}$ the random variable given by

$$
n_{0}(\omega):=\sharp\left(\omega \cap \left[-\frac{1}{2}, \frac{1}{2}[) \quad \forall \omega \in \mathfrak{O} .\right.\right.
$$

The following theorem is proved in [10]:

THEOREM 3.4. Under the assumptions stated above, there exists a sequence of reals $\left(\varepsilon_{k}\right)$ converging to 0 and a $P$-negligible subset $\mathfrak{N}$ of $\mathfrak{O}$, such that for all $\omega \in \mathfrak{O} \backslash \mathfrak{N}$,

$$
n_{\varepsilon_{k}}(\omega) \stackrel{\star}{\longrightarrow} E_{P}^{\mathcal{F}} n_{0}(\omega) \quad \text { weakly* in } L^{\infty}(\Omega) .
$$

The following result straightforwardly follows from theorems 3.1, 3.4:

TheOrem 3.5. Assume (3.11), (3.12), and let $\left(\varepsilon_{k}\right)$ and $\mathfrak{N}$ be the sequence and the P-negligible set given by Theorem 3.4. Then, for all $\omega \in \mathfrak{O} \backslash \mathfrak{N}$, the solution to $\left(\mathcal{P}_{\varepsilon_{k}}(\omega)\right)$, weakly* converges in $L^{\infty}\left(0, T ; H_{0}^{1}\left(\Omega ; \mathbb{R}^{3}\right)\right)$ to the unique solution to the problem $\left(\mathcal{P}_{\left(E_{P}^{\mathcal{F}} n_{0}(\omega), k, \kappa\right)}^{\text {hom }}\right)$ defined by (3.18-3.21).

REMARK 3.6. The restriction of the Hausdorff distance $d_{\mathcal{H}}$ to $\mathfrak{O}$ is an extended metric on $\mathfrak{O}$, and the mapping $d_{\mathfrak{O}}: \mathfrak{O}^{2} \rightarrow[0,1]$ defined by $d_{\mathfrak{O}}\left(\omega, \omega^{\prime}\right):=$ $\min \left\{1, d_{\mathcal{H}}\left(\omega, \omega^{\prime}\right)\right\}$ is a finite metric on $\mathfrak{O}$ which turns $\mathfrak{O}$ into a complete metric space. 
3.3. Intermediate case . Under the assumptions

$$
\begin{aligned}
& \varepsilon^{2} \ll \mu_{0 \varepsilon} \ll 1, \quad 0 \leq \lambda_{0 \varepsilon} \leq C \mu_{0 \varepsilon}, \\
& n_{\varepsilon} \rightarrow n \quad \text { strongly in } \quad L^{2}(\Omega),
\end{aligned}
$$

and (3.17) (see Remark 3.9), we show that the solution to (3.5) weakly* converges in $L^{\infty}\left(0, T ; L^{2}\left(\Omega ; \mathbb{R}^{3}\right)\right)$ to the unique solution to $\left(\mathcal{P}_{(n, k, \kappa)}^{h o m}\right)$ defined by

$$
\left(\begin{array} { c } 
{ ( \mathcal { P } _ { ( n , k , 0 ) } ^ { \text { hom } } ) : } \\
{ ( 0 < k < + \infty ) }
\end{array} \left\{\begin{array}{c}
\left(\rho(1-\vartheta n)+n \bar{\rho}_{1}\right) \frac{\partial^{2} \boldsymbol{u}}{\partial t^{2}}-n k \operatorname{div} \boldsymbol{\sigma}_{x^{\prime}}\left(\boldsymbol{u}^{\prime}\right) \\
=\left(\rho(1-\vartheta n)+n \bar{\rho}_{1}\right) \boldsymbol{f} \quad \text { in } \Omega \times(0, T), \\
u_{1}, u_{2} \in C\left([0, T] ; L_{n}^{2}\left(0, L ; H_{0}^{1}\left(\Omega^{\prime}\right)\right)\right), \\
\boldsymbol{u} \in C^{1}\left([0, T] ; L^{2}\left(\Omega ; \mathbb{R}^{3}\right)\right), \boldsymbol{u}(0)=\boldsymbol{a}_{0}, \frac{\partial \boldsymbol{u}}{\partial t}(0)=\boldsymbol{b}_{0},
\end{array}\right.\right.
$$

$\left(\mathcal{P}_{(n,+\infty, 0)}^{h o m}\right):\left\{\begin{array}{l}\left(\rho(1-\vartheta n)+n \bar{\rho}_{1}\right) \frac{\partial^{2} u_{3}}{\partial t^{2}}=\left(\rho(1-\vartheta n)+n \bar{\rho}_{1}\right) f_{3} \quad \text { in } \Omega \times(0, T), \\ \rho \frac{\partial^{2} u_{\alpha}}{\partial t^{2}}=\rho f_{\alpha} \text { in } \widehat{\{n=0\}} \times(0, T),(\alpha \in\{1,2\}), \\ n u_{1}=n u_{2}=0, \quad \boldsymbol{u} \in C^{1}\left([0, T] ; L^{2}\left(\Omega ; \mathbb{R}^{3}\right)\right), \\ \boldsymbol{u}(0)=\left(a_{01} \mathbb{1}_{\{n=0\}}, a_{02} \mathbb{1}_{\{n=0\}}, a_{03}\right), \\ \frac{\partial \boldsymbol{u}}{\partial t}(0)=\left(b_{01} \mathbb{1}_{\{n=0\}}, b_{02} \mathbb{1}_{\{n=0\}}, b_{03}\right),\end{array}\right.$

$$
\begin{aligned}
& \left(\mathcal{P}_{(n,+\infty, \kappa)}^{h o m}\right):\left\{\begin{array}{l}
\left(\rho(1-\vartheta n)+n \bar{\rho}_{1}\right) \frac{\partial^{2} u_{3}}{\partial t^{2}}+n \frac{\kappa}{3} \frac{l+1}{l+2} \sum_{\alpha, \beta=1}^{2} \frac{\partial^{4} u_{3}}{\partial x_{\alpha}^{2} \partial x_{\beta}^{2}} \\
=\left(\rho(1-\vartheta n)+n \bar{\rho}_{1}\right) f_{3} \text { in } \Omega \times(0, T), \\
\rho \frac{\partial^{2} u_{\alpha}}{\partial t^{2}}=\rho f_{\alpha} \text { in } \widehat{\{n=0\}} \times(0, T),(\alpha \in\{1,2\}), \\
n u_{1}=n u_{2}=0, \\
u_{3} \in C\left([0, T] ; L_{n}^{2}\left(0, L ; H_{0}^{2}\left(\Omega^{\prime}\right)\right)\right), \boldsymbol{u} \in C^{1}\left([0, T] ; L^{2}\left(\Omega ; \mathbb{R}^{3}\right)\right), \\
\boldsymbol{u}(0)=\left(a_{01} \mathbb{1}_{\{n=0\}}, a_{02} \mathbb{1}_{\{n=0\}}, a_{03}\right), \\
\frac{\partial \boldsymbol{u}}{\partial t}(0)=\left(b_{01} \mathbb{1}_{\{n=0\}}, b_{02} \mathbb{1}_{\{n=0\}}, b_{03}\right),
\end{array}\right. \\
& \left(\mathcal{P}_{(n,+\infty,+\infty)}^{\text {hom }}\right):\left\{\begin{array}{l}
\rho \frac{\partial^{2} \boldsymbol{u}}{\partial t^{2}}=\rho \boldsymbol{f} \quad \text { in } \widehat{\{n=0\}} \times(0, T), \\
n \boldsymbol{u}=0, \quad \boldsymbol{u} \in C^{1}\left([0, T] ; L^{2}\left(\Omega ; \mathbb{R}^{3}\right)\right), \\
\boldsymbol{u}(0)=\boldsymbol{a}_{0} \mathbb{1}_{\{n=0\}}, \frac{\partial \boldsymbol{u}}{\partial t}(0)=\boldsymbol{b}_{0} \mathbb{1}_{\{n=0\}} .
\end{array}\right.
\end{aligned}
$$


THEOREM 3.7. Under (3.17), (3.26), (3.27), the solution to (3.5) weakly* converges in $L^{\infty}\left(0, T ; L^{2}\left(\Omega ; \mathbb{R}^{3}\right)\right)$ to the unique solution to $\left(\mathcal{P}_{(n, k, \kappa)}^{\text {hom }}\right)$ given by (3.283.31).

REMARK 3.8. (i) Problems (3.28-3.31) are formally deduced from (3.18-3.21) by removing the term "div $\boldsymbol{\sigma}(\boldsymbol{u}) "$ (see (3.11)). This indicates that no strain energy is stored in the softer phase.

(ii) Assumption (3.27), stronger than (3.16), precludes the application of Theorem 3.4 and the extension of Theorem 3.7 to the setting of stochastic homogenization.

REMARK 3.9. If (3.17) is not satisfied, the sequence $\left(\boldsymbol{u}_{\varepsilon}\right)$ of the solutions to (3.5) weakly* converges in $L^{\infty}\left(0, T ; L^{2}\left(\Omega ; \mathbb{R}^{3}\right)\right)$ to the unique solution of the variational problem (4.23), (6.60), (6.61). Under (3.17), this variational problem is equivalent to (3.29-3.31).

REMARK 3.10. Theorems 3.1, 3.7 can be extended to the case of a multiphase stratified elastic composite comprising several distributions of stiff layers of various stiffness and thickness (see Remark 6.2 for more details). Combining our results with those established in [8], [13], [14], one can also derive the effective equations governing the behavior of a composite comprising disjoint distributions of stiff layers alternating with softer interlayers in which fibers or grain-like inclusions are embedded (see also Remark 3.17).

REMARK 3.11. Under the assumptions of theorems 3.1, 3.7, the limit problem associated to the sequence of equilibrium problems

$$
-\operatorname{div}\left(\boldsymbol{\sigma}_{\varepsilon}\left(\boldsymbol{u}_{\varepsilon}\right)\right)=\boldsymbol{f} \quad \text { in } \quad \Omega, \quad \boldsymbol{u}_{\varepsilon} \in H_{0}^{1}\left(\Omega, \mathbb{R}^{3}\right), \quad \boldsymbol{f} \in L^{2}\left(\Omega, \mathbb{R}^{3}\right),
$$

is given by

$$
\boldsymbol{u} \in V \quad \text { and } \quad a(\boldsymbol{u}, \boldsymbol{w})=(\boldsymbol{f}, \boldsymbol{w})_{H}, \quad \forall \boldsymbol{w} \in V
$$

where the Hilbert spaces $V$ and $H$ and the non-negative symmetric bilinear form a $(.,$. are specified in Remark 6.3. Under the assumptions of Theorem 3.7, this bilinear form fails to be coercive on $L^{2}\left(\Omega ; \mathbb{R}^{3}\right)$ if $\kappa=0$ and may fail to be so if the function $n$ is not bounded from below by a positive constant. Remark 6.3 states conditions ensuring coerciveness in stratified multiphase media. Coerciveness can also be achieved by reinforcing the composite by stiff fibers embedded in the soft phase (see [8, Proposition 5.2]).

3.4. Case of soft interlayers with Lamé coefficients of order $\varepsilon^{2}$. We assume that

$$
\mu_{0 \varepsilon}=\varepsilon^{2} \mu_{0}, \quad \lambda_{0 \varepsilon}=\varepsilon^{2} \lambda_{0}, \quad \mu_{0}>0, \quad \lambda_{0} \geq 0
$$

and that the stiff layers are periodically distributed (see (3.15)):

$$
B_{\varepsilon}:=\bigcup_{i \in Z_{\varepsilon}} B_{\varepsilon}^{i} ; \quad B_{\varepsilon}^{i}:=\Omega^{\prime} \times\left(\varepsilon i+r_{\varepsilon} I\right) .
$$

Under these hypotheses, setting (see Remark 3.13) 


$$
\begin{aligned}
& Y:=\left(-\frac{1}{2}, \frac{1}{2}\right)^{3} ; B:=\left(-\frac{1}{2}, \frac{1}{2}\right)^{2} \times\left(-\frac{\vartheta}{2}, \frac{\vartheta}{2}\right) ; \Sigma:=\left(-\frac{1}{2}, \frac{1}{2}\right)^{2} \times\{0\} \\
& A:=B \quad \text { if } \vartheta>0, \quad A:=\Sigma \quad \text { if } \vartheta=0, \\
& \vartheta>0 \quad \text { if } \kappa=0,
\end{aligned}
$$

and denoting by $\mathcal{M}\left(\bar{\Omega} ; \mathbb{R}^{3}\right)$ the set of $\mathbb{R}^{3}$-valued Radon measures on $\bar{\Omega}$, we show that the solution $\boldsymbol{u}_{\varepsilon}$ to $(3.5)$ two-scale converges to $\boldsymbol{u}_{0} \in C\left([0, T] ; L^{2}\left(\Omega, H_{\sharp}^{1}\left(Y ; \mathbb{R}^{3}\right)\right)\right.$ ) (see Section 4 for the definition of this convergence), and the sequence $\left(\boldsymbol{u}_{\varepsilon} m_{\varepsilon}\right)$, where $m_{\varepsilon}$ is the measure defined by

$$
m_{\varepsilon}:=\frac{\varepsilon}{r_{\varepsilon}} \mathbb{1}_{B_{\varepsilon}}(x) \mathcal{L}_{\lfloor\Omega}^{3}
$$

weakly* converges in $L^{\infty}\left(0, T ; \mathcal{M}\left(\bar{\Omega} ; \mathbb{R}^{3}\right)\right)$ to $\boldsymbol{v} \in C^{1}\left([0, T] ; L^{2}\left(\Omega ; \mathbb{R}^{3}\right)\right)$, where $\left(\boldsymbol{u}_{0}, \boldsymbol{v}\right)$ is the unique solution to the coupled system of equations (comparable in certain respects with $[8,(2.17)])$

$$
\left\{\begin{array}{l}
\left(\mathcal{P}_{\text {soft }}^{\text {hom }}\right) \\
\left(\mathcal{P}_{\text {stiff }}^{\text {hom }}(k, \kappa)\right),
\end{array}\right.
$$

defined below in terms of $k, \kappa$, and $\vartheta$ given, respectively, by (3.8) and (3.10). The fields $\boldsymbol{u}_{0}$ and $\boldsymbol{v}$ are linked by the following relation in $\Omega \times(0, T) \times A$ :

$$
\boldsymbol{v}(x, t)=\boldsymbol{u}_{0}(x, t, y) \quad \text { in } \Omega \times(0, T) \times A .
$$

We introduce the operators $\boldsymbol{e}_{y}, \boldsymbol{\sigma}_{0 y}: H^{1}\left(Y ; \mathbb{R}^{3}\right) \rightarrow L^{2}\left(Y ; \mathbb{S}^{3}\right), \boldsymbol{g}: \mathcal{H} \rightarrow \mathbb{R}^{3}$ defined by

$$
\begin{aligned}
& \left(\boldsymbol{e}_{y}(\boldsymbol{w})\right)_{i j}=\frac{1}{2}\left(\frac{\partial w_{i}}{\partial y_{j}}+\frac{\partial w_{j}}{\partial y_{i}}\right), \boldsymbol{\sigma}_{0 y}(\boldsymbol{w}):=\lambda_{0} \operatorname{tr}\left(\boldsymbol{e}_{y}(\boldsymbol{w})\right) \boldsymbol{I}+2 \mu_{0} \boldsymbol{e}_{y}(\boldsymbol{w}), \\
& \boldsymbol{g}(\boldsymbol{w}):=\left\{\begin{array}{l}
-\int_{\partial(Y \backslash B) \cap \bar{B}} \boldsymbol{\sigma}_{0 y}(\boldsymbol{w}) \boldsymbol{\nu}_{Y \backslash B} d \mathcal{H}^{2}(y), \quad \text { if } A=B, \\
\int_{\Sigma}\left(\boldsymbol{\sigma}_{0 y}\left(\boldsymbol{w}^{+}\right)-\boldsymbol{\sigma}_{0 y}\left(\boldsymbol{w}^{-}\right)\right) \cdot \boldsymbol{e}_{3} d \mathcal{H}^{2}(y) \quad \text { if } A=\Sigma,
\end{array}\right.
\end{aligned}
$$

where $\boldsymbol{\nu}_{Y \backslash B}$ stands for the outward normal to $\partial(Y \backslash B)$ and

$$
\mathcal{H}:=\left\{\boldsymbol{w} \in H^{1}\left(Y \backslash A ; \mathbb{R}^{3}\right), \operatorname{div}\left(\boldsymbol{\sigma}_{0 y}(\boldsymbol{w})\right) \in\left(H^{1}\left(Y \backslash A ; \mathbb{R}^{3}\right)\right)^{\prime}\right\},
$$

denoting by $E^{\prime}$ the topological dual of a Banach space $E$, and by $\boldsymbol{w}^{+}$(resp. $\boldsymbol{w}^{-}$) the restriction of $\boldsymbol{w}$ to $\left(\frac{-1}{2}, \frac{1}{2}\right)^{2} \times\left(0, \frac{1}{2}\right)$ (resp. $\left.\left(\frac{-1}{2}, \frac{1}{2}\right)^{2} \times\left(\frac{-1}{2}, 0\right)\right)$. Problem $\left(\mathcal{P}_{\text {soft }}^{\text {hom }}\right)$ in (3.40) is the equation of $\boldsymbol{u}_{0}$ in $\Omega \times(0, T) \times(Y \backslash A)$ coupled with $\boldsymbol{v}$ through (3.41) and given by (denoting by $\boldsymbol{\nu}$ the outward normal to $\partial Y$ ): 


$$
\left(\mathcal{P}_{\text {soft }}^{\text {hom }}\right):\left\{\begin{array}{l}
\rho \frac{\partial^{2} \boldsymbol{u}_{0}}{\partial t^{2}}-\operatorname{div}_{y}\left(\boldsymbol{\sigma}_{0 y}\left(\boldsymbol{u}_{0}\right)\right)=\rho \boldsymbol{f} \quad \text { in } \Omega \times(0, T) \times Y \backslash A, \\
\left(\boldsymbol{u}_{0}, \boldsymbol{v}\right) \quad \text { satisfies } \quad(3.41), \\
\boldsymbol{\sigma}_{0 y}\left(\boldsymbol{u}_{0}\right) \boldsymbol{\nu}(y)=-\boldsymbol{\sigma}_{0 y}\left(\boldsymbol{u}_{0}\right) \boldsymbol{\nu}(-y) \text { on } \Omega \times(0, T) \times \partial Y, \\
\boldsymbol{u}_{0} \in C\left([0, T] ; L^{2}\left(\Omega, H_{\sharp}^{1}\left(Y ; \mathbb{R}^{3}\right)\right)\right) \cap C^{1}\left([0, T] ; L^{2}\left(\Omega \times Y ; \mathbb{R}^{3}\right)\right), \\
\boldsymbol{u}_{0}(0) \mathbb{1}_{Y \backslash A}=\boldsymbol{a}_{0} \mathbb{1}_{Y \backslash A}, \quad \frac{\partial \boldsymbol{u}_{0}}{\partial t}(0) \mathbb{1}_{Y \backslash A}=\boldsymbol{b}_{0} \mathbb{1}_{Y \backslash A} .
\end{array}\right.
$$

Equation (3.44) governs the effective behavior of the displacement in the soft phase. Problem $\left(\mathcal{P}_{\text {stiff }}^{\text {hom }}(k, \kappa)\right)$ in (3.40) is an equation of $\boldsymbol{v}$ in $\Omega \times(0, T)$ coupled with $\left(\mathcal{P}_{\text {soft }}^{\text {hom }}\right)$ through the source term $\boldsymbol{g}\left(\boldsymbol{u}_{0}\right)$ defined by (3.42). This equation rules the effective behavior of the displacement in the stiff layers. Its form is determined by the order of magnitude of the coefficients $k, \kappa$. If $0<k<+\infty$, we get (see (2.1))

$$
\left(\mathcal{P}_{\text {stiff }}^{\text {hom }}(k, 0)\right):\left\{\begin{array}{l}
\bar{\rho}_{1} \frac{\partial^{2} \boldsymbol{v}}{\partial t^{2}}-k \operatorname{div} \boldsymbol{\sigma}_{x^{\prime}}\left(\boldsymbol{v}^{\prime}\right)=\bar{\rho}_{1} \boldsymbol{f}+\boldsymbol{g}\left(\boldsymbol{u}_{0}\right) \text { in } \Omega \times(0, T), \\
v_{1}, v_{2} \in C\left([0, T] ; L^{2}\left(0, L ; H_{0}^{1}\left(\Omega^{\prime}\right)\right)\right) \cap C^{1}\left([0, T] ; L^{2}(\Omega)\right), \\
\boldsymbol{v} \in C^{1}\left([0, T] ; L^{2}\left(\Omega ; \mathbb{R}^{3}\right)\right), \quad \boldsymbol{v}(0)=\boldsymbol{a}_{0}, \frac{\partial \boldsymbol{v}}{\partial t}(0)=\boldsymbol{b}_{0} .
\end{array}\right.
$$

If $(k, \kappa)=(+\infty, 0)$, we obtain

$$
\left(\mathcal{P}_{\text {stiff }}^{\text {hom }}(+\infty, 0)\right):\left\{\begin{array}{l}
\bar{\rho}_{1} \frac{\partial^{2} v_{3}}{\partial t^{2}}=\bar{\rho}_{1} f_{3}+\left(\boldsymbol{g}\left(\boldsymbol{u}_{0}\right)\right)_{3} \quad \text { in } \Omega \times(0, T) \\
v_{1}=v_{2}=0, \\
v_{3} \in C^{1}\left([0, T], L^{2}(\Omega)\right), v_{3}(0)=a_{03}, \frac{\partial v_{3}}{\partial t}(0)=b_{03} .
\end{array}\right.
$$

If $0<\kappa<+\infty$, the emergence of fourth derivatives of $v_{3}$ reveal bending effects:

$$
\left(\mathcal{P}_{\text {stiff }}^{\text {hom }}(+\infty, \kappa)\right):\left\{\begin{array}{l}
\bar{\rho}_{1} \frac{\partial^{2} v_{3}}{\partial t^{2}}+\frac{\kappa}{3} \frac{l+1}{l+2} \sum_{\alpha, \beta=1}^{2} \frac{\partial^{4} v_{3}}{\partial x_{\alpha}^{2} \partial x_{\beta}^{2}} \\
=\bar{\rho}_{1} f_{3}+\left(\boldsymbol{g}\left(\boldsymbol{u}_{0}\right)\right)_{3} \quad \text { in } \Omega \times(0, T), \\
v_{1}=v_{2}=0, \\
v_{3} \in C\left([0, T] ; L^{2}\left(0, L ; H_{0}^{2}\left(\Omega^{\prime}\right)\right)\right) \cap C^{1}\left([0, T] ; L^{2}(\Omega)\right), \\
v_{3}(0)=a_{03}, \frac{\partial v_{3}}{\partial t}(0)=b_{03} .
\end{array}\right.
$$

If $\kappa=+\infty$, the displacement in the stiff layers asymptotically vanishes:

$$
\left(\mathcal{P}_{\text {stiff }}^{\text {hom }}(+\infty,+\infty)\right): \quad \boldsymbol{v}=0 .
$$

THEOREM 3.12. Under (3.34), (3.35), the solution $\boldsymbol{u}_{\varepsilon}$ to (3.5) two-scale converges to $\boldsymbol{u}_{0}$ with respect to $x$ and weakly* converges in $L^{\infty}\left(0, T ; L^{2}\left(\Omega, \mathbb{R}^{3}\right)\right)$ to $\boldsymbol{u}=$ 
$\int_{Y} \boldsymbol{u}_{0}(., y) d y$, and the sequence $\left(\boldsymbol{u}_{\varepsilon} m_{\varepsilon}\right)$, where $m_{\varepsilon}$ is defined by (3.39), weakly* converges in $L^{\infty}\left(0, T ; \mathcal{M}\left(\bar{\Omega}, \mathbb{R}^{3}\right)\right)$ to $\boldsymbol{v} \mathcal{L}_{[\Omega}^{3}$, where $\left(\boldsymbol{u}_{0}, \boldsymbol{v}\right)$ is the unique solution to (3.40). Moreover, $\boldsymbol{u}_{\varepsilon}(\tau)$ two-scale converges to $\boldsymbol{u}_{0}(\tau)$ with respect to $x$, for each $\tau \in[0, T]$.

REMARK 3.13. When $\vartheta=0, k \in(0,+\infty]$, additional sub-regimes are likely to appear in the cases $r_{\varepsilon}=c \varepsilon^{2}$ and $\varepsilon^{2} \ll r_{\varepsilon} \ll \varepsilon$, possibly attended by the emergence of bending effects. Similar phenomena have been studied by V. V. Zhikov [55] and V. V. Zhikov and S. E. Pastukhova [56] in the context of periodic singular structures.

REMARK 3.14. One can show (see [8, p.2548] for more details), that if $\boldsymbol{a}_{0}=0$ and if the fields $\boldsymbol{b}_{0}, \boldsymbol{f}$ are sufficiently regular, the following corrector result holds

$$
\lim _{\varepsilon \rightarrow 0}\left\|\boldsymbol{u}_{\varepsilon}-\boldsymbol{u}_{0}\left(x, t, \frac{x}{\varepsilon}\right)\right\|_{L^{2}\left(\Omega \times(0, T) ; \mathbb{R}^{3}\right)}=0 .
$$

Remark 3.15. The effective problem (3.40) is non-local in space and time. Nonlocal effects [3], [5]-[19], [20, 21, 23, 34, 44], [47]-[50], and memory effects [1, 4, 37, $53]$ are typical of composite media with high contrast.

REMARK 3.16 (Multiphase stratified elastic media). In the same way as in [8, Section 4], we can extend Theorem 3.12 to the case of a multiphase medium whereby $m \varepsilon$-periodic disconnected families $B_{\varepsilon}^{[1]}, \ldots, B_{\varepsilon}^{[m]}$ of parallel layers are embedded in a soft matrix. The limit problem then takes the following form

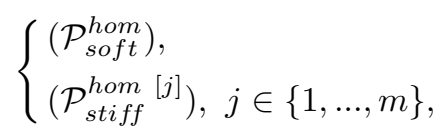

and can be written under the variational form (4.23) for some suitable choice of data $H, V, a, h, \xi_{0}, \xi_{1}$. Each family $B_{\varepsilon}^{[m]}$ is associated to some subset $A^{[j]}$ of $Y$ like in (3.37). The system $\left(\mathcal{P}_{\text {soft }}^{\text {hom }}\right)$ governs the effective displacement in the soft phase, and only differs from (3.44) by the relation (3.41) which is replaced by a series of relations on each set $\Omega \times(0, T) \times A^{[j]}$ between $\boldsymbol{u}_{0}$ and some auxiliary variable $\boldsymbol{v}^{[j]}$ characterizing the effective displacement in $B_{\varepsilon}^{[j]}$. Each problem $\left(\mathcal{P}_{\text {stiff }}^{\text {hom }}{ }^{[j]}\right)$ consists of an equation of $\boldsymbol{v}^{[j]}$ of the same form as $\left(\mathcal{P}_{\text {stiff }}^{\text {hom }}\right)$ in (3.40), coupled with $\boldsymbol{u}_{0}$ through the operator $\boldsymbol{g}^{[j]}$ deduced from (3.42) by replacing $A$ by $A^{[j]}$.

REMARK 3.17. Multiphase composites comprising, besides stiff layers, periodic distributions of fibers or grain-like inclusions, can also be considered. By way of illustration, let us examine the case of a composite consisting of an $\varepsilon$-periodic family $B_{\varepsilon}^{[1]}$ of parallel stiff layers of thickness $\vartheta \varepsilon(\vartheta>0)$ alternating with a softer medium in which an $\varepsilon$-periodic family $B_{\varepsilon}^{[2]}$ of parallel stiff fibers and an $\varepsilon$-periodic family $B_{\varepsilon}^{[3]}$ of stiff grain-like inclusions are embedded. The disconnected families $\left(B_{\varepsilon}^{[i]}\right)$ are defined in terms of three connected subsets $B^{[1]}, B^{[2]}, B^{[3]}$ of $Y$ with disjoint closure by setting $B_{\varepsilon}^{[i]}:=\varepsilon\left(\bigcup_{j \in \mathbb{Z}^{3}} j+B^{[i]}\right) \cap \Omega$ (see figure 3.2). We assume that the Lamé coefficients take constant values of order at least 1 in each $B_{\varepsilon}^{[i]}$, and constant values $\varepsilon^{2} \lambda_{0}, \varepsilon^{2} \mu_{0}$ in $\Omega \backslash \bigcup_{i=1}^{3} B_{\varepsilon}^{[i]}$. The limit problem then takes the form

$$
\left\{\begin{array}{l}
\left(\mathcal{P}_{\text {soft }}^{\text {hom }}\right), \\
\left(\mathcal{P}_{\text {stiff }}^{\text {hom }}[i]\right), i \in\{1,2,3\},
\end{array}\right.
$$

where, setting $B:=\bigcup_{i=1}^{3} B^{[i]}, \boldsymbol{y}_{B^{[i]}}:=\frac{1}{\left|B^{[i]}\right|} \int_{B^{[i]}} \boldsymbol{y} d y$, 


$$
\left(\mathcal{P}_{\text {soft }}^{\text {hom }}\right):\left\{\begin{array}{lr}
\rho \frac{\partial^{2} \boldsymbol{u}_{0}}{\partial t^{2}}-\operatorname{div}_{y}\left(\boldsymbol{\sigma}_{0 y}\left(\boldsymbol{u}_{0}\right)\right)=\rho \boldsymbol{f} & \text { in } \Omega \times(0, T) \times(Y \backslash B), \\
\boldsymbol{u}_{0}(x, t, y)=\boldsymbol{v}^{[1]}(x, t) & \text { in } \quad \Omega \times(0, T) \times B^{[1]}, \\
\boldsymbol{u}_{0}=\boldsymbol{v}^{[i]}+\boldsymbol{r}^{[i]} \wedge\left(\boldsymbol{y}-\boldsymbol{y}_{B}[i]\right) & \text { in } \quad \Omega \times(0, T) \times B^{[i]},(j \in\{2,3\}), \\
\boldsymbol{\sigma}_{0 y}\left(\boldsymbol{u}_{0}\right) \cdot \boldsymbol{n}(y)=-\boldsymbol{\sigma}_{0 y}\left(\boldsymbol{u}_{0}\right) \cdot \boldsymbol{n}(-y) & \text { on } \Omega \times(0, T) \times \partial Y, \\
\boldsymbol{u}_{0} \in C\left([0, T] ; L^{2}\left(\Omega, H_{\sharp}^{1}\left(Y ; \mathbb{R}^{3}\right)\right)\right) \cap C^{1}\left([0, T] ; L^{2}\left(\Omega \times Y ; \mathbb{R}^{3}\right)\right), \\
\boldsymbol{u}_{0}(0) 1_{Y \backslash B}=\boldsymbol{a}_{0} 1_{Y \backslash B}, \quad \frac{\partial \boldsymbol{u}_{0}}{\partial t}(0) 1_{Y \backslash B}=\boldsymbol{b}_{0} 1_{Y \backslash B} .
\end{array}\right.
$$

The problem $\left(\mathcal{P}_{\text {stiff }}^{\text {hom }}{ }^{[1]}\right)$ in (3.50) is an equation of $\boldsymbol{v}^{[1]}$ characterizing the effective displacement in the stiff layers as described in Remark 3.16. For $i \in\{2,3\}$, the problem $\left(\mathcal{P}_{\text {stiff }}^{\text {hom }}{ }^{[i]}\right)$ is a system of equations of $\boldsymbol{v}^{[i]}, \boldsymbol{r}^{[i]}$ defined in [8, Section 4], governing the effective displacement in the fibers if $i=2$, in the grain-like inclusions if $i=3$. The couple $\left(\boldsymbol{v}^{[i]}, \frac{1}{\varepsilon} \boldsymbol{r}^{[i]}\right)$ represents the reduction elements (on the principal axes of the fibers or at the geometrical center of gravity of the inclusions) of an helicoidal vector field locally approximating the displacement in the fibers or the grain-like inclusions.

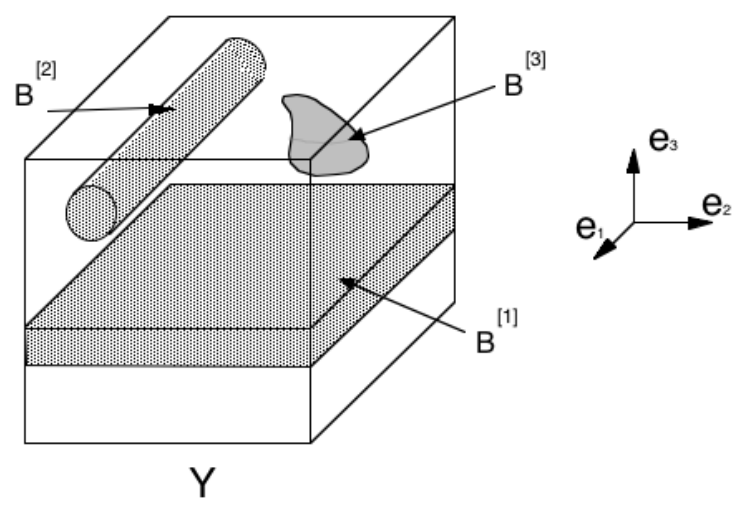

FIG. 3.2.

REMARK 3.18. The solution to (3.44) is actually independent of $y_{1}, y_{2}$. We have $\boldsymbol{u}_{0}(x, y)=\check{\boldsymbol{u}}_{0}\left(x, y_{3}\right)$, where, setting $I:=\left(-\frac{1}{2}, \frac{1}{2}\right), \check{\boldsymbol{u}}_{0}\left(x, y_{3}\right)$ is the unique solution to

$$
\left(\mathcal{P}_{\text {soft }}^{\text {hom }}\right):\left\{\begin{array}{l}
\rho \frac{\partial^{2} \check{\boldsymbol{u}}_{0}}{\partial t^{2}}-\left(\begin{array}{ccc}
\mu & 0 & 0 \\
0 & \mu & 0 \\
0 & 0 & \lambda+2 \mu
\end{array}\right) \frac{\partial^{2} \check{\boldsymbol{u}}_{0}}{\partial y_{3}^{2}}=\rho \boldsymbol{f} \quad \text { in } \Omega \times(0, T) \times I \backslash\left[-\frac{\vartheta}{2}, \frac{\vartheta}{2}\right], \\
\boldsymbol{v}(x, t)=\check{\boldsymbol{u}}_{0}\left(x, t, y_{3}\right) \quad \text { in } \Omega \times(0, T) \times\left[-\frac{\vartheta}{2}, \frac{\vartheta}{2}\right], \\
\frac{\partial \check{\boldsymbol{u}}_{0}}{\partial y_{3}}\left(., \frac{1}{2}\right)=\frac{\partial \check{\boldsymbol{u}}_{0}}{\partial y_{3}}\left(.,-\frac{1}{2}\right), \\
\check{\boldsymbol{u}}_{0} \in C\left([0, T] ; L^{2}\left(\Omega, H_{\sharp}^{1}\left(I ; \mathbb{R}^{3}\right)\right)\right) \cap C^{1}\left([0, T] ; L^{2}\left(\Omega \times I ; \mathbb{R}^{3}\right)\right), \\
\check{\boldsymbol{u}}_{0}(0) \mathbb{1}_{I \backslash\left[-\frac{\vartheta}{2}, \frac{\vartheta}{2}\right]}=\boldsymbol{a}_{0} \mathbb{1}_{I \backslash\left[-\frac{\vartheta}{2}, \frac{\vartheta}{2}\right]}, \quad \frac{\partial \check{\boldsymbol{u}}_{0}}{\partial t}(0) \mathbb{1}_{I \backslash\left[-\frac{\vartheta}{2}, \frac{\vartheta}{2}\right]}=\boldsymbol{b}_{0} \mathbb{1}_{I \backslash\left[-\frac{\vartheta}{2}, \frac{\vartheta}{2}\right]} .
\end{array}\right.
$$


We retained the dependence on $y_{1}, y_{2}$ in (3.44) in order to extend our results to such multiphase media as described in Remark 3.17 and derive (3.50).

REMARK 3.19 (Equilibrium equations). The conclusions of [8, Corollary 5.1] remain valid in the stratified case: if the solution to the equilibrium problem (3.32) two-scale converges to $\boldsymbol{u}_{0} \in L^{2}\left(\Omega \times Y ; \mathbb{R}^{3}\right)$, then $\boldsymbol{u}_{0}$ is a solution to the problem

$$
\boldsymbol{u}_{0} \in V \quad \text { and } \quad a\left(\boldsymbol{u}_{0}, \boldsymbol{w}_{0}\right)=\left(\boldsymbol{f}, \boldsymbol{w}_{0}\right)_{H}, \quad \forall \boldsymbol{w}_{0} \in V,
$$

where $V, H, a(.,$.$) are specified in Remark 3.16. The form a(.,.) may fail to be$ coercive on $L^{2}\left(\Omega \times Y ; \mathbb{R}^{3}\right)$, and problem (3.51) may then have no solution. The same conditions stated in Remark 6.3 for Theorem 3.7 also ensure the coercivity of a (.,.) in the present context. Coerciveness is also achieved in the composite described in Remark 3.17 provided the Lamé coefficients of the fibers are at least of order $\frac{1}{\varepsilon^{2}}$ (see [8, Proposition 5.2]). Note in passing that one should substitute 1 for $\rho_{\varepsilon}$ in [8, Formula 5.1], otherwise the proof of "(iii) $\Rightarrow(i v) "$ in [8, p. 2552] is false.

REMARK 3.20. Under Assumption (3.34), the slightest perturbation of periodicity leads to a complete change of the form of the effective problem. For instance, if $m \in \mathbb{N}$ and $\omega_{\varepsilon}^{j}=\varepsilon\left(j+\frac{1}{2}\right)$ if $j$ is a multiple of $m$, and $\omega_{\varepsilon}^{j}=\varepsilon j$ otherwise in (3.35), then the limit problem is a system of equations coupling $\boldsymbol{u}_{0}$ with $m$ auxiliary variables $\boldsymbol{v}^{[1]}, \ldots, \boldsymbol{v}^{[m]}$ as described in Remark 3.16, which can not be expressed, as in theorems 3.1, 3.7, simply in terms of the function $n$ defined by (3.16). The extension of Theorem 3.12 to the non-periodic case is far beyond the scope of this paper.

4. Two-scale convergence and other analysis tools. In Section 4.1, we recall some properties of the two-scale convergence of G. Allaire [3] and G. Nguetseng [41] and reproduce some statements of [8] in a suitable form for the present context. In Section 4.2, we introduce a non-periodic notion of two-scale convergence with respect to a sequence of measures and establish a compactness result (Lemma 4.2). Two classical analysis results are recalled in Section 4.3.

4.1. Two-scale convergence. A sequence $\left(f_{\varepsilon}\right)$ in $L^{2}\left(0, T ; L^{2}(\Omega)\right)$ is said to two-scale converge to $f_{0} \in L^{2}\left(0, T ; L^{2}(\Omega \times Y)\right)$ with respect to $x$ if, for all $\varphi_{0} \in$ $\mathcal{D}\left(\Omega \times(0, T), C_{\sharp}^{\infty}(Y)\right)$,

$$
\begin{aligned}
& \lim _{\varepsilon \rightarrow 0} \int_{\Omega \times(0, T)} f_{\varepsilon}(x, t) \varphi_{0}\left(x, t, \frac{x}{\varepsilon}\right) d x d t=\int_{\Omega \times(0, T) \times Y} f_{0} \varphi_{0} d x d t d y, \\
& \text { (notation: } \left.f_{\varepsilon} \longrightarrow f_{0}\right) .
\end{aligned}
$$

A sequence $\left(\varphi_{\varepsilon}\right) \subset L^{2}\left(0, T ; L^{2}(\Omega)\right)$ strongly two-scale converges to $\varphi_{0} \in L^{2}\left(0, T ; L^{2}(\Omega\right.$ $\times Y)$ ) with respect to $x$ if

$$
\begin{aligned}
& \varphi_{\varepsilon} \longrightarrow \varphi_{0} \quad \text { and } \quad \lim _{\varepsilon \rightarrow 0}\left\|\varphi_{\varepsilon}\right\|_{L^{2}\left(0, T ; L^{2}(\Omega)\right)}=\left\|\varphi_{0}\right\|_{L^{2}\left(0, T ; L^{2}(\Omega \times Y)\right)}, \\
& \text { (notation: } \left.\varphi_{\varepsilon} \longrightarrow \varphi_{0}\right) .
\end{aligned}
$$

The symbols $\longrightarrow$ and $\longrightarrow$ will also denote the two-scale convergence and the strong two-scale convergence of sequences $\left(f_{\varepsilon}\right)$ in $L^{2}(\Omega)$ independent of $t$, defined by formally considering them as constant in $t$. Any bounded sequence in $L^{2}\left(0, T ; L^{2}(\Omega)\right)$ has a 
two-scale convergent subsequence [41]. An admissible sequence with respect to twoscale convergence is a sequence $\left(\varphi_{\varepsilon}\right) \subset L^{2}\left(0, T ; L^{2}(\Omega)\right)$ that two-scale converges to some $\varphi_{0} \in L^{2}\left(0, T ; L^{2}(\Omega \times Y)\right)$ and such that, for every two-scale convergent sequence $\left(f_{\varepsilon}\right)$,

$$
\lim _{\varepsilon \rightarrow 0} \int_{\Omega \times(0, T)} f_{\varepsilon} \varphi_{\varepsilon} d x d t=\int_{\Omega \times(0, T) \times Y} f_{0} \varphi_{0} d x d t d y .
$$

A sequence $\left(\varphi_{\varepsilon}\right)$ is admissible if and only if it strongly two-scale converges to some $\varphi_{0}$ (see [8, p.2528]). For all $\psi_{0} \in L^{2}\left(0, T ; L^{2}\left(\Omega, C_{\sharp}^{\infty}(Y)\right)\right) \cup L_{\sharp}^{2}(Y, C(\overline{\Omega \times(0, T)})$ ), the sequence $\left(\psi_{0}\left(x, t, \frac{x}{\varepsilon}\right)\right)_{\varepsilon>0}$ strongly two-scale converges to $\psi_{0}$ (see [3], Lemma 5.2, Corollary 5.4). In particular, if $Q$ is a Borel subset of $Y$, and $Q_{\sharp}$ is its periodization on $\mathbb{R}^{3}$ defined by $(2.2)$, then the sequence $\left(\mathbb{1}_{Q_{\sharp}}\left(\frac{x}{\varepsilon}\right)\right)$ strongly two-scale converges to $\mathbb{1}_{Q}(y)$. Under (3.35), if $\vartheta>0$, then $\left|\mathbb{1}_{\Omega \backslash B_{\varepsilon}}-\mathbb{1}_{(Y \backslash B)_{\sharp}}\left(\frac{x}{\varepsilon}\right)\right|_{L^{2}(\Omega)} \rightarrow 0$, therefore $\mathbb{1}_{\Omega \backslash B_{\varepsilon}} \longrightarrow \mathbb{1}_{Y \backslash B}$. If $\vartheta=0,\left(\mathbb{1}_{\Omega \backslash B_{\varepsilon}}\right)$ strongly converges to 1 in $L^{2}(\Omega)$, hence strongly two-scale converges to 1 . We deduce that (see (3.37))

$$
\mathbb{1}_{\Omega \backslash B_{\varepsilon}} \longrightarrow \mathbb{1}_{Y \backslash A}, \quad \mathbb{1}_{B_{\varepsilon}} \longrightarrow \mathbb{1}_{A} .
$$

The next Lemma is a straightforward variant of [8, Lemma 6.1].

Lemma 4.1. (i) Let $\left(h_{\varepsilon}\right)$ be a bounded sequence in $L^{\infty}(\Omega \times(0, T) \times Y)$ such that $h_{\varepsilon} \longrightarrow h_{0}$. Then, for every sequence $\left(\chi_{\varepsilon}\right) \subset L^{2}\left(0, T ; L^{2}(\Omega)\right)$, the following implications hold:

$$
\begin{aligned}
& \chi_{\varepsilon} \longrightarrow \chi_{0} \Longrightarrow \chi_{\varepsilon} h_{\varepsilon} \longrightarrow \chi_{0} h_{0}, \\
& \chi_{\varepsilon} \longrightarrow \chi_{0} \Longrightarrow \chi_{\varepsilon} h_{\varepsilon} \longrightarrow \chi_{0} h_{0} .
\end{aligned}
$$

(ii) If $\left(f_{\varepsilon}\right)$ is bounded in $L^{\infty}\left(0, T ; L^{2}(\Omega)\right)$, then $\left(f_{\varepsilon}\right)$ two-scale converges, up to a subsequence, to some $f_{0} \in L^{\infty}\left(0, T ; L^{2}(\Omega \times Y)\right)$. If in addition $\left(f_{\varepsilon}\right)$ is bounded in $W^{1, \infty}\left(0, T ; L^{2}(\Omega)\right)$, then $f_{0} \in W^{1, \infty}\left(0, T ; L^{2}(\Omega \times Y)\right)$ and $\left(\frac{\partial f_{\varepsilon}}{\partial t}\right)$ two-scale converges to $\frac{\partial f_{0}}{\partial t}$. Besides, if $f_{\varepsilon}(0) \longrightarrow a_{0}$, then $a_{0}=f_{0}(0)$ and $f_{\varepsilon}(\tau) \longrightarrow f_{0}(\tau), \forall \tau \in[0, T]$. Furthermore, if $\left(\frac{\partial f_{\varepsilon}}{\partial t}\right) \longrightarrow \frac{\partial f_{0}}{\partial t}$ and $f_{\varepsilon}(0) \longrightarrow a_{0}$, then $f_{\varepsilon}(\tau) \longrightarrow f_{0}(\tau), \forall \tau \in[0, T]$.

4.2. Two-scale convergence with respect to $\left(m_{\varepsilon}\right)$. One can easily check that the sequence $\left(m_{\varepsilon}\right)$ defined by (3.39) is bounded in $\mathcal{M}(\bar{\Omega})$ and satisfies

$$
m_{\varepsilon} \stackrel{*}{\rightarrow} n \mathcal{L}_{\lfloor\Omega}^{3} \quad \text { weakly* in } \mathcal{M}(\bar{\Omega}),
$$

where $n$ is defined by (3.16). Notice that

$$
n=1 \quad \text { under } \quad(3.35) \text {. }
$$

In what follows, the symbol $L_{n}^{2}\left(\Omega ; \mathbb{R}^{3}\right)$ stands for the set of all Borel fields $\boldsymbol{w}: \Omega \rightarrow \mathbb{R}^{3}$ such that $\int_{\Omega}|\boldsymbol{w}|^{2} n d x<+\infty$. Similarly, for any Hilbert space $H$, we denote by $L_{n}^{2}(0, L ; H)$ the set of all Borel fields $\boldsymbol{w}:(0, L) \rightarrow H$ such that $\int_{0}^{L}|\boldsymbol{w}|_{H}^{2} n d x<+\infty$. We set (see (3.3), (3.4))

$$
y_{\varepsilon}(z):=\sum_{j \in J_{\varepsilon}}\left(z-\omega_{\varepsilon}^{j}\right) \mathbb{1}_{\omega_{\varepsilon}^{j}+r_{\varepsilon}(1+\delta) I}(z) .
$$


We say that a sequence $\left(f_{\varepsilon}\right)$ in $L^{2}\left(0, T ; L^{2}(\Omega)\right)$ two-scale converges to $f_{0} \in L^{2}(0, T$; $\left.L_{n}^{2}(\Omega \times I)\right)$ with respect to the sequence of measures $\left(m_{\varepsilon}\right)$ if for each $\psi \in \mathcal{D}(\Omega \times(0, T)$; $\left.C_{\sharp}^{\infty}(I)\right)$, the following holds

$$
\lim _{\varepsilon \rightarrow 0} \int_{\Omega \times(0, T)} f_{\varepsilon}(x, t) \psi\left(x, t, \frac{y_{\varepsilon}\left(x_{3}\right)}{r_{\varepsilon}}\right) d m_{\varepsilon} d t=\int_{\Omega \times(0, T) \times I} f_{0} \psi n d x d t d y_{3} .
$$

(Notation: $\left.f_{\varepsilon} \stackrel{m_{\varepsilon}}{ } f_{0}\right)$.

Lemma 4.2. Let $\left(f_{\varepsilon}\right)$ be a sequence in $L^{2}\left(0, T ; L^{2}(\Omega)\right)$ satisfying

$$
\sup _{\varepsilon>0} \int_{\Omega \times(0, T)}\left|f_{\varepsilon}\right|^{2} d m_{\varepsilon} d t<+\infty .
$$

Then $\left(f_{\varepsilon}\right)$ two-scale converges with respect to $\left(m_{\varepsilon}\right)$, up to a subsequence, to some $f_{0} \in L_{n}^{2}\left(0, T ; L^{2}(\Omega \times I)\right)$. In addition, if

$$
\sup _{\varepsilon>0, \tau>0} \int_{\Omega}\left|f_{\varepsilon}\right|^{2}(\tau) d m_{\varepsilon}<+\infty
$$

then $f_{0} \in L^{\infty}\left(0, T ; L_{n}^{2}(\Omega \times I)\right)$.

Proof. By Cauchy-Schwarz Inequality and by (4.11), we have

$$
\begin{aligned}
& \mid \int_{\Omega \times(0, T)} f_{\varepsilon}(x, t) \psi\left(x, t, \frac{y_{\varepsilon}\left(x_{3}\right)}{r_{\varepsilon}}\right) d m_{\varepsilon} d t \mid \\
& \leq C\left(\int_{\Omega \times(0, T)}\left|f_{\varepsilon}\right|^{2} d m_{\varepsilon} d t\right)^{\frac{1}{2}}\|\psi\|_{L^{\infty}(\Omega \times(0, T) \times I)} \\
& \leq C\|\psi\|_{L^{\infty}(\Omega \times(0, T) \times I)} \quad \forall \psi \in C(\overline{\Omega \times(0, T) \times I}) .
\end{aligned}
$$

Hence, by the Riesz representation theorem, for each $\varepsilon>0$ there exists a finite Radon measure $\theta_{\varepsilon} \in \mathcal{M}(\overline{\Omega \times(0, T) \times I})$ such that

$$
\int \psi d \theta_{\varepsilon}=\int_{\Omega \times(0, T)} f_{\varepsilon}(x, t) \psi\left(x, t, \frac{y_{\varepsilon}\left(x_{3}\right)}{r_{\varepsilon}}\right) d m_{\varepsilon} d t \quad \forall \psi \in C(\overline{\Omega \times(0, T) \times I}) .
$$

By (4.13) and (4.14), the sequence $\left(\theta_{\varepsilon}\right)$ is bounded in $\mathcal{M}(\overline{\Omega \times(0, T) \times I})$, thus weakly* converges, up to a subsequence, to some $\theta \in \mathcal{M}(\overline{\Omega \times(0, T) \times I})$. By Cauchy-Schwarz inequality, we have

$$
\left|\int \psi d \theta_{\varepsilon}\right| \leq\left(\int_{\Omega \times(0, T)}\left|f_{\varepsilon}\right|^{2} d m_{\varepsilon} d t\right)^{\frac{1}{2}}\left(\int_{\Omega \times(0, T)}\left|\psi\left(x, t, \frac{y_{\varepsilon}\left(x_{3}\right)}{r_{\varepsilon}}\right)\right|^{2} d m_{\varepsilon} d t\right)^{\frac{1}{2}}
$$

The proof of the next statement is similar to that of [3, Lemma 1.3]:

$$
\lim _{\varepsilon \rightarrow 0} \int_{\Omega}\left|\varphi\left(x, \frac{y_{\varepsilon}\left(x_{3}\right)}{r_{\varepsilon}}\right)\right|^{2} d m_{\varepsilon}=\int_{\Omega \times I}|\varphi|^{2} n d x d y_{3} \quad \forall \varphi \in C(\overline{\Omega \times I}) .
$$


We deduce from (4.11), (4.14), (4.15), (4.16), and from the weak* convergence in $\mathcal{M}(\overline{\Omega \times(0, T) \times I})$ of $\left(\theta_{\varepsilon}\right)$ to $\theta$, that

$$
\left|\int \psi d \theta\right|=\lim _{\varepsilon \rightarrow 0}\left|\int \psi d \theta_{\varepsilon}\right| \leq C\|\psi\|_{L_{n}^{2}(\Omega \times(0, T) \times I)} \quad \forall \psi \in C(\overline{\Omega \times(0, T) \times I}) .
$$

Thus, the linear form $\psi \rightarrow \int \psi d \theta$ is continuous on $C(\overline{\Omega \times(0, T) \times I})$ with respect to the strong topology of $L_{n}^{2}(\Omega \times(0, T) \times I)$. By a density argument, this linear form can be extended to a continuous linear form on $L_{n}^{2}(\Omega \times(0, T) \times I)$ which, by the Riesz representation theorem, takes the form $\psi \rightarrow \int_{\Omega \times(0, T) \times I} \psi f_{0} n d x d t d y$ for some $f_{0} \in L_{n}^{2}(\Omega \times(0, T) \times I)$. We infer that $\theta=n f_{0} \mathcal{L}_{\lfloor\Omega \times(0, T) \times I}^{5}$, and then, taking (4.14) and the weak* convergence of $\left(\theta_{\varepsilon}\right)$ to $\theta$ into account, deduce (4.10). Under (4.12), by Fubini's Theorem and Cauchy-Schwarz inequality, we have

$$
\int_{\Omega \times(0, T)} f_{\varepsilon}(x, t) \psi\left(x, t, \frac{y_{\varepsilon}\left(x_{3}\right)}{r_{\varepsilon}}\right) d m_{\varepsilon} d t \leq C \int_{(0, T)}\left(\int\left|\psi\left(x, \tau, \frac{y_{\varepsilon}\left(x_{3}\right)}{r_{\varepsilon}}\right)\right|^{2} d m_{\varepsilon}\right)^{\frac{1}{2}} d \tau .
$$

By passing to the limit as $\varepsilon \rightarrow 0$ in the last inequality, thanks to (4.16) and to the Dominated Convergence Theorem, we get $\int_{\Omega \times(0, T) \times I} f_{0} \psi n d x d t d y_{3} \leq C|\psi|_{L^{1}\left(0, T ; L_{n}^{2}(\Omega \times I)\right)}$ and deduce, by the arbitrary choice of $\psi$, that $f_{0} \in L^{\infty}\left(0, T ; L_{n}^{2}(\Omega \times I)\right)$.

4.3. Two classical results. For the reader's convenience, we reproduce below Lemma A2 of [11] (see also [17] for a more general version) and Theorem 6.2 of [8], which collects some abstract results proved in $[25,35,36]$. The lemma will be employed to identify the limit of the sequence $\left(\boldsymbol{u}_{\varepsilon} m_{\varepsilon}\right)$, where $\boldsymbol{u}_{\varepsilon}$ is the solution to (3.5). The theorem will be applied to check the existence, the uniqueness, and some regularity properties of the solution to Problem (3.5) and of the associated limit problems. In what follows, we denote by $E^{\prime}$ the continuous dual space of a Banach space $E$ and by $\sigma\left(E^{\prime}, E\right)$ the weak* topology on $E^{\prime}$.

LEMma 4.3. Let $K$ be a compact subset of $\mathbb{R}^{N}$ and $\left(\theta_{\varepsilon}\right)$ a bounded sequence of positive Radon measures on $K$, weakly* converging in $\mathcal{M}(K)$ to some $\theta \in \mathcal{M}(K)$. Let $\left(f_{\varepsilon}\right)$ be a sequence of $\theta_{\varepsilon}$-measurable functions such that $\sup _{\varepsilon} \int\left|f_{\varepsilon}\right|^{2} d \theta_{\varepsilon}<+\infty$. Then the sequence $\left(f_{\varepsilon} \theta_{\varepsilon}\right)$ is sequentially relatively compact in the weak ${ }^{*}$ topology $\sigma(\mathcal{M}(K), C(K))$

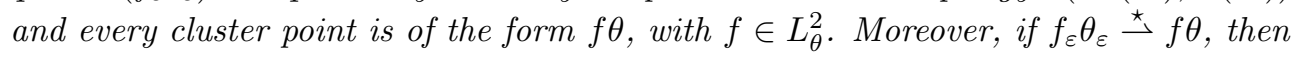

$$
\liminf _{\varepsilon \rightarrow 0} \int\left|f_{\varepsilon}\right|^{2} d \theta_{\varepsilon} \geq \int|f|^{2} d \theta
$$

TheOREM 4.4. Let $V$ and $H$ be separable Hilbert spaces such that $V \subset H=$ $H^{\prime} \subset V^{\prime}$, with continuous and dense imbeddings. Let $\|.\|_{V},|\cdot|_{H},((., .))_{V},(., .)_{H}$ denote their respective norm and inner product. Let $a: V \times V \rightarrow \mathbb{R}$ be a continuous bilinear symmetric form on $V$. Let $A \in \mathcal{L}\left(V, V^{\prime}\right)$ be defined by $a(\xi, \widetilde{\xi})=$ $(A \xi, \widetilde{\xi})_{\left(V^{\prime}, V\right)}, \forall(\xi, \widetilde{\xi}) \in V^{2}$. Assume that

$$
\exists(\lambda, \alpha) \in[0,+\infty) \times(0,+\infty), \quad a(\xi, \xi)+\lambda|\xi|_{H}^{2} \geq \alpha\|\xi\|_{V}^{2}, \quad \forall \xi \in V
$$


Let $h \in L^{2}(0, T ; H), \xi_{0} \in V, \xi_{1} \in H$. Then there exists a unique solution $\xi$ to

$$
\begin{aligned}
& A \xi(t)+\frac{\partial^{2} \xi}{\partial t^{2}}(t)=h(t), \quad \xi \in L^{2}(0, T ; V), \\
& \frac{\partial \xi}{\partial t} \in L^{2}(0, T ; H), \quad \xi(0)=\xi_{0}, \quad \frac{\partial \xi}{\partial t}(0)=\xi_{1} .
\end{aligned}
$$

Furthermore, we have

$$
\xi \in C([0, T] ; V) \cap C^{1}([0, T] ; H), \quad \frac{\partial \xi}{\partial t} \in L^{2}(0, T ; V), \quad \frac{\partial^{2} \xi}{\partial t^{2}} \in L^{2}\left(0, T ; V^{\prime}\right) .
$$

Besides, setting

$$
e(\tau):=\frac{1}{2}\left[\left(\frac{\partial \xi}{\partial t}(\tau), \frac{\partial \xi}{\partial t}(\tau)\right)_{H}+a(\xi(\tau), \xi(\tau))\right], \forall \tau \in[0, T]
$$

the following holds

$$
e(\tau)=e(0)+\int_{0}^{\tau}\left(h, \frac{\partial \xi}{\partial t}\right)_{H} d t, \quad \forall \tau \in[0, T] .
$$

Problem (4.19) is equivalent to

$$
\begin{gathered}
\int_{0}^{T}\left(a(\xi(t), \widetilde{\xi}) \eta(t)+(\xi(t), \widetilde{\xi})_{H} \frac{\partial^{2} \eta}{\partial t^{2}}(t)\right) d t+\left(\xi_{0}, \widetilde{\xi}\right)_{H} \frac{\partial \eta}{\partial t}(0) \\
-\left(\xi_{1}, \widetilde{\xi}\right)_{H} \eta(0)=\int_{0}^{T}(h, \widetilde{\xi})_{H} \eta(t) d t \\
\forall \widetilde{\xi} \in V, \quad \forall \eta \in \mathcal{D}(]-\infty, T[) ; \quad \xi \in L^{2}(0, T ; V), \quad \frac{\partial \xi}{\partial t} \in L^{2}(0, T ; H) .
\end{gathered}
$$

5. Asymptotic behavior of the solution to (3.5). In this section, we establish a series of estimates satisfied by the solution $\boldsymbol{u}_{\varepsilon}$ to (3.5) (see Proposition 5.2), and investigate in lemmas 5.3, 5.4 , and 5.5, the asymptotic behavior of sequences satisfying such estimates. These results are synthetized in Corollary 5.6. We start with a key inequality.

LEMMA 5.1. We have

$$
\int\left(\left|\frac{\varphi_{1}}{r_{\varepsilon}}\right|^{2}+\left|\frac{\varphi_{2}}{r_{\varepsilon}}\right|^{2}+\left|\varphi_{3}\right|^{2}\right) d m_{\varepsilon} \leq \frac{C}{r_{\varepsilon}^{2}} \int|\boldsymbol{e}(\boldsymbol{\varphi})|^{2} d m_{\varepsilon} \quad \forall \boldsymbol{\varphi} \in H_{0}^{1}\left(\Omega ; \mathbb{R}^{3}\right) .
$$

Proof. By (3.4) and (3.39), it is sufficient to show that for all $j \in J_{\varepsilon}$, and all $\varphi \in H^{1}\left(B_{\varepsilon}^{j} ; \mathbb{R}^{3}\right)$ such that $\varphi=0$ on $\partial B_{\varepsilon}^{j} \cap \partial \Omega$,

$$
\int_{B_{\varepsilon}^{j}}\left(\left|\frac{\varphi_{1}}{r_{\varepsilon}}\right|^{2}+\left|\frac{\varphi_{2}}{r_{\varepsilon}}\right|^{2}+\left|\varphi_{3}\right|^{2}\right) d x \leq \frac{C}{r_{\varepsilon}^{2}} \int_{B_{\varepsilon}^{j}}|\boldsymbol{e}(\boldsymbol{\varphi})|^{2} d x
$$

By Korn's inequality, we have 


$$
\begin{aligned}
& \int_{\Omega^{\prime} \times\left(-\frac{1}{2} ; \frac{1}{2}\right)}|\boldsymbol{\psi}|^{2} d y \leq C \int_{\Omega^{\prime} \times\left(-\frac{1}{2} ; \frac{1}{2}\right)}|\boldsymbol{e}(\boldsymbol{\psi})|^{2} d y \quad \forall \boldsymbol{\psi} \in W, \\
& W:=\left\{\boldsymbol{\psi} \in H^{1}\left(\Omega^{\prime} \times\left(-\frac{1}{2} ; \frac{1}{2}\right) ; \mathbb{R}^{3}\right), \quad \boldsymbol{\psi}=0 \text { on } \partial \Omega^{\prime} \times\left(-\frac{1}{2} ; \frac{1}{2}\right)\right\} .
\end{aligned}
$$

By making the change of variable $y=\left(x_{1}, x_{2}, \frac{x_{3}-\omega_{\varepsilon}^{j}}{r_{\varepsilon}}\right)$, we get, for all $\boldsymbol{\psi} \in W$,

$$
\int_{B_{\varepsilon}^{j}}|\boldsymbol{\psi}|^{2}\left(x_{1}, x_{2}, \frac{x_{3}-\omega_{\varepsilon}^{j}}{r_{\varepsilon}}\right) d x \leq C \int_{B_{\varepsilon}^{j}}|\boldsymbol{e}(\boldsymbol{\psi})|^{2}\left(x_{1}, x_{2}, \frac{x_{3}-\omega_{\varepsilon}^{j}}{r_{\varepsilon}}\right) d x
$$

Setting $\varphi(x)=\left(\begin{array}{c}\psi_{1} \\ \psi_{2} \\ \frac{1}{r_{\varepsilon}} \psi_{3}\end{array}\right)\left(x_{1}, x_{2}, \frac{x_{3}-\omega_{\varepsilon}^{j}}{r_{\varepsilon}}\right)$, a straightforward computation yields

$$
\begin{aligned}
& \int_{B_{\varepsilon}^{j}}\left(\left|\varphi_{1}\right|^{2}+\right.\left.\left|\varphi_{2}\right|^{2}+r_{\varepsilon}^{2}\left|\varphi_{3}\right|^{2}\right)(x) d x=\int_{B_{\varepsilon}^{j}}|\boldsymbol{\psi}|^{2}\left(x_{1}, x_{2}, \frac{x_{3}-\omega_{\varepsilon}^{j}}{r_{\varepsilon}}\right) d x \\
& \leq C \int_{B_{\varepsilon}^{j}}|\boldsymbol{e}(\boldsymbol{\psi})|^{2}\left(x_{1}, x_{2}, \frac{x_{3}-\omega_{\varepsilon}^{j}}{r_{\varepsilon}}\right) d x \leq C \int_{B_{\varepsilon}^{j}}|\boldsymbol{e}(\boldsymbol{\varphi})|^{2}(x) d x .
\end{aligned}
$$

The inequality (5.2) is proved. $\square$

Proposition 5.2. There exists a unique solution $\boldsymbol{u}_{\varepsilon}$ to (3.5). Moreover,

$$
\frac{\partial \boldsymbol{u}_{\varepsilon}}{\partial t} \in L^{2}\left(0, T ; H_{0}^{1}\left(\Omega ; \mathbb{R}^{3}\right)\right), \frac{\partial^{2} \boldsymbol{u}_{\varepsilon}}{\partial t^{2}} \in L^{2}\left(0, T ; H^{-1}\left(\Omega ; \mathbb{R}^{3}\right)\right) .
$$

Under (3.6), (3.9), there exists a constant $C>0$ such that

$$
\begin{array}{ll}
\int_{\Omega} \mu_{0 \varepsilon}\left|\boldsymbol{e}\left(\boldsymbol{u}_{\varepsilon}\right)\right|^{2}(\tau) d x+\int_{\Omega}\left(\rho_{\varepsilon}\left|\frac{\partial \boldsymbol{u}_{\varepsilon}}{\partial t}\right|^{2}+\left|\boldsymbol{u}_{\varepsilon}\right|^{2}\right)(\tau) d x \leq C & \forall \tau \in[0, T], \\
\int\left|\boldsymbol{e}\left(\boldsymbol{u}_{\varepsilon}\right)\right|^{2}(\tau)+\left|\boldsymbol{u}_{\varepsilon}^{\prime}\right|^{2}(\tau) d m_{\varepsilon} \leq C\left(\frac{r_{\varepsilon}}{\varepsilon} \mu_{1 \varepsilon}\right)^{-1} & \forall \tau \in[0, T], \\
\int\left|u_{\varepsilon 3}\right|^{2}(\tau) d m_{\varepsilon} \leq C\left(\frac{r_{\varepsilon}^{3}}{\varepsilon} \mu_{1 \varepsilon}\right)^{-1}, \quad \int\left|\boldsymbol{u}_{\varepsilon}\right|^{2}(\tau) d m_{\varepsilon} \leq C & \forall \tau \in[0, T] .
\end{array}
$$

Proof. Problem (3.5) is equivalent to $(4.23)$, where $H:=L^{2}\left(\Omega ; \mathbb{R}^{3}\right),(\boldsymbol{\xi}, \widetilde{\boldsymbol{\xi}})_{H}:=$ $\int_{\Omega} \rho_{\varepsilon} \boldsymbol{\xi} \cdot \widetilde{\boldsymbol{\xi}} d x, V:=H_{0}^{1}\left(\Omega ; \mathbb{R}^{3}\right)\left(V^{\prime}=H^{-1}\left(\Omega ; \mathbb{R}^{3}\right)\right), a(\boldsymbol{\xi}, \widetilde{\boldsymbol{\xi}}):=\int_{\Omega} \boldsymbol{\sigma}_{\varepsilon}(\boldsymbol{\xi}): e(\widetilde{\boldsymbol{\xi}}) d x$, and $\left(\boldsymbol{\xi}_{0}, \boldsymbol{\xi}_{1}, \boldsymbol{h}\right)=\left(\boldsymbol{a}_{0}, \boldsymbol{b}_{0}, \boldsymbol{f}\right)$. By $(3.7),\left(H,(., .)_{H}\right)$ is a Hilbert space and the assumptions of Theorem 4.4 are satisfied. Therefore, Problem (3.5) has a unique solution. Assertion (5.4) follows from (4.20). By (4.22) we have, for all $\tau \in[0, T]$,

$$
\begin{aligned}
\frac{1}{2} \int_{\Omega}\left(\rho_{\varepsilon}\left|\frac{\partial \boldsymbol{u}_{\varepsilon}}{\partial t}\right|^{2}+\boldsymbol{\sigma}_{\varepsilon}\left(\boldsymbol{u}_{\varepsilon}\right): \boldsymbol{e}\left(\boldsymbol{u}_{\varepsilon}\right)\right)(\tau) d x \\
\quad=\frac{1}{2} \int_{\Omega}\left(\rho_{\varepsilon}\left|\boldsymbol{b}_{0}\right|^{2}+\boldsymbol{\sigma}_{\varepsilon}\left(\boldsymbol{a}_{0}\right): \boldsymbol{e}\left(\boldsymbol{a}_{0}\right)\right) d x+\int_{\Omega \times(0, \tau)} \rho_{\varepsilon} \boldsymbol{f} \cdot \frac{\partial \boldsymbol{u}_{\varepsilon}}{\partial t} d x d t .
\end{aligned}
$$


We deduce from Cauchy-Schwarz Inequality that

$$
\left|\int_{\Omega \times(0, \tau)} \rho_{\varepsilon} \boldsymbol{f} \cdot \frac{\partial \boldsymbol{u}_{\varepsilon}}{\partial t} d x d t\right| \leq \sqrt{\int_{\Omega \times(0, T)} \rho_{\varepsilon}|\boldsymbol{f}|^{2} d x d t} \sqrt{\int_{\Omega \times(0, T)} \rho_{\varepsilon}\left|\frac{\partial \boldsymbol{u}_{\varepsilon}}{\partial t}\right|^{2} d x d t} .
$$

Taking (3.9) into account, we infer

$$
\begin{aligned}
& \int_{\Omega}\left(\rho_{\varepsilon}\left|\frac{\partial \boldsymbol{u}_{\varepsilon}}{\partial t}\right|^{2} d x+\boldsymbol{\sigma}_{\varepsilon}\left(\boldsymbol{u}_{\varepsilon}\right): \boldsymbol{e}\left(\boldsymbol{u}_{\varepsilon}\right)\right)(\tau) d x \\
& \leq C\left(1+\sqrt{\int_{\Omega \times(0, T)} \rho_{\varepsilon}\left|\frac{\partial \boldsymbol{u}_{\varepsilon}}{\partial t}\right|^{2} d x d t}\right) \quad \forall \tau \in[0, T]
\end{aligned}
$$

By integrating (5.6) with respect to $\tau$ over $(0, T)$, we deduce that $\int_{\Omega \times(0, T)} \rho_{\varepsilon}\left|\frac{\partial \boldsymbol{u}_{\varepsilon}}{\partial t}\right|^{2}$ $d x d t \leq C$ and then, coming back to (5.6), that

$$
\int_{\Omega} \rho_{\varepsilon}\left|\frac{\partial \boldsymbol{u}_{\varepsilon}}{\partial t}\right|^{2}(\tau) d x+\int_{\Omega} \boldsymbol{\sigma}_{\varepsilon}\left(\boldsymbol{u}_{\varepsilon}\right): \boldsymbol{e}\left(\boldsymbol{u}_{\varepsilon}\right)(\tau) d x \leq C \quad \forall \tau \in[0, T] .
$$

We infer from (3.5), (3.6), (3.39), and (5.7), that

$$
\begin{array}{ll}
\int_{\Omega} \rho_{\varepsilon}\left|\frac{\partial \boldsymbol{u}_{\varepsilon}}{\partial t}\right|^{2}(\tau)+\mu_{0 \varepsilon}\left|\boldsymbol{e}\left(\boldsymbol{u}_{\varepsilon}\right)\right|^{2}(\tau) d x \leq C & \forall \tau \in[0, T], \\
\int\left|\boldsymbol{e}\left(\boldsymbol{u}_{\varepsilon}\right)\right|^{2}(\tau) d m_{\varepsilon} \leq C\left(\frac{r_{\varepsilon}}{\varepsilon} \mu_{1 \varepsilon}\right)^{-1} & \forall \tau \in[0, T] .
\end{array}
$$

By (3.7), (3.39), and (5.8), and by the continuity of $\boldsymbol{a}_{0}$ (see (3.5)), we have

$$
\begin{gathered}
\int_{\Omega}\left|\boldsymbol{u}_{\varepsilon}\right|^{2}(\tau) d x+\int\left|\boldsymbol{u}_{\varepsilon}\right|^{2}(\tau) d m_{\varepsilon}=\int_{\Omega}\left|\boldsymbol{a}_{0}+\int_{0}^{\tau} \frac{\partial \boldsymbol{u}_{\varepsilon}}{\partial t}(t) d t\right|^{2} d\left(\mathcal{L}^{3}+m_{\varepsilon}\right)(x) \\
\leq C\left(1+\int_{\Omega \times(0, T)} \rho_{\varepsilon}\left|\frac{\partial \boldsymbol{u}_{\varepsilon}}{\partial t}\right|^{2}(t) d x d t\right) \leq C \quad \forall \tau \in[0, T] .
\end{gathered}
$$

By (5.1) and (5.8), we have

$$
\int\left|\frac{u_{\varepsilon 1}}{r_{\varepsilon}}\right|^{2}+\left|\frac{u_{\varepsilon 2}}{r_{\varepsilon}}\right|^{2}+\left|u_{\varepsilon 3}\right|^{2}(\tau) d m_{\varepsilon} \leq \frac{C}{r_{\varepsilon}^{2}} \int\left|\boldsymbol{e}\left(\boldsymbol{u}_{\varepsilon}\right)\right|^{2}(\tau) d m_{\varepsilon} \leq \frac{C \varepsilon}{r_{\varepsilon}^{3} \mu_{1 \varepsilon}} \quad \forall \tau \in[0, T],
$$

which, combined with (5.8), (5.9) completes the proof of (5.5). $\square$

LEMma 5.3. Let $\left(\boldsymbol{u}_{\varepsilon}\right)$ be a sequence in $W^{1, \infty}\left(0, T ; H_{0}^{1}\left(\Omega ; \mathbb{R}^{3}\right), L^{2}\left(\Omega ; \mathbb{R}^{3}\right)\right)$ satisfying

$$
\sup _{\varepsilon>0, \tau \in(0, T)} \int\left|\boldsymbol{u}_{\varepsilon}\right|^{2}+\left|\frac{\partial \boldsymbol{u}_{\varepsilon}}{\partial t}\right|^{2}+\left|\boldsymbol{e}\left(\boldsymbol{u}_{\varepsilon}\right)\right|^{2}(\tau) d m_{\varepsilon}<+\infty
$$

Then, up to a subsequence, the following holds 


$$
\begin{array}{ll}
\boldsymbol{u}_{\varepsilon} m_{\varepsilon} \stackrel{\star}{\rightarrow} n \boldsymbol{v}, \quad \frac{\partial \boldsymbol{u}_{\varepsilon}}{\partial t} m_{\varepsilon} \stackrel{\star}{\rightarrow} n \frac{\partial \boldsymbol{v}}{\partial t} & \text { weakly*in } L^{\infty}\left(0, T ; \mathcal{M}\left(\bar{\Omega} ; \mathbb{R}^{3}\right)\right), \\
v_{1}, v_{2} \in L^{\infty}\left(0, T ; L_{n}^{2}\left(0, L ; H_{0}^{1}\left(\Omega^{\prime}\right)\right)\right), & \boldsymbol{v} \in W^{1, \infty}\left(0, T ; L_{n}^{2}\left(\Omega ; \mathbb{R}^{3}\right)\right), \\
\boldsymbol{e}_{x^{\prime}}\left(\boldsymbol{u}_{\varepsilon}^{\prime}\right) m_{\varepsilon} \stackrel{\star}{\rightarrow} n \boldsymbol{e}_{x^{\prime}}\left(\boldsymbol{v}^{\prime}\right) & \text { weakly*in } L^{\infty}\left(0, T ; \mathcal{M}\left(\bar{\Omega} ; \mathbb{S}^{3}\right)\right) .
\end{array}
$$

Furthermore,

$$
\begin{array}{ll}
n v_{1}=n v_{2}=0 \quad \text { if } \quad & \liminf _{\varepsilon \rightarrow 0} \sup _{\tau \in(0, T)} \int\left|\boldsymbol{e}\left(\boldsymbol{u}_{\varepsilon}\right)\right|^{2}(\tau) d m_{\varepsilon}=0, \\
n \boldsymbol{v}=0 & \text { if } \quad \liminf _{\varepsilon \rightarrow 0} \sup _{\tau \in(0, T)} \int\left|\frac{1}{r_{\varepsilon}} \boldsymbol{e}\left(\boldsymbol{u}_{\varepsilon}\right)\right|^{2}(\tau) d m_{\varepsilon}=0 .
\end{array}
$$

Moreover, if

$$
\begin{aligned}
& \sup _{\varepsilon>0, \tau \in(0, T)} \int_{\Omega} \mu_{0 \varepsilon}\left|\boldsymbol{e}\left(\boldsymbol{u}_{\varepsilon}\right)\right|^{2}(\tau) d x<+\infty, \quad \varepsilon^{2} \ll \mu_{\varepsilon 0}, \quad \text { and } \\
& n_{\varepsilon} \rightarrow n \text { strongly in } L^{2}(\Omega) \text { and } \boldsymbol{u}_{\varepsilon} \stackrel{\star}{\rightarrow} \boldsymbol{u} \text { weakly* in } L^{\infty}\left(0, T ; L^{2}\left(\Omega ; \mathbb{R}^{3}\right)\right),
\end{aligned}
$$

or if

$$
\begin{aligned}
& \sup _{\varepsilon>0, \tau \in(0, T)} \int_{\Omega}\left|\boldsymbol{e}\left(\boldsymbol{u}_{\varepsilon}\right)\right|^{2}(\tau) d x<+\infty, \quad \text { and } \\
& \boldsymbol{u}_{\varepsilon} \rightarrow \boldsymbol{u} \text { strongly in } L^{2}\left(0, T ; L^{2}\left(\Omega ; \mathbb{R}^{3}\right)\right),
\end{aligned}
$$

then $n \boldsymbol{u}=n \boldsymbol{v}$.

Proof. By applying Lemma 4.3 to $\theta_{\varepsilon}:=\mathcal{L}_{[(0, T)}^{1} \otimes m_{\varepsilon}, K:=\overline{(0, T) \times \Omega}, f_{\varepsilon} \in$ $\left\{\boldsymbol{u}_{\varepsilon}, \frac{\partial \boldsymbol{u}_{\varepsilon}}{\partial t}, \boldsymbol{e}_{x^{\prime}}\left(\boldsymbol{u}_{\varepsilon}^{\prime}\right)\right\}$, taking (4.7) and (5.10) into account, we obtain the convergences

$$
\begin{array}{ll}
\boldsymbol{u}_{\varepsilon} m_{\varepsilon} \stackrel{\star}{\rightarrow} n \boldsymbol{v}, \quad \frac{\partial \boldsymbol{u}_{\varepsilon}}{\partial t} m_{\varepsilon} \stackrel{\star}{\rightarrow} n \boldsymbol{\gamma} & \text { weakly* in } \left.\mathcal{M}\left(\overline{(0, T) \times \Omega} ; \mathbb{R}^{3}\right)\right), \\
\boldsymbol{e}_{x^{\prime}}\left(\boldsymbol{u}_{\varepsilon}^{\prime}\right) m_{\varepsilon} \stackrel{\star}{\rightarrow} n \boldsymbol{\Xi} & \text { weakly* in } \left.\mathcal{M}\left(\overline{(0, T) \times \Omega} ; \mathbb{S}^{3}\right)\right),
\end{array}
$$

up to a subsequence, for some suitable $\boldsymbol{v}, \boldsymbol{\gamma} \in L^{2}\left(0, T ; L_{n}^{2}\left(\Omega ; \mathbb{R}^{3}\right)\right), \boldsymbol{\Xi} \in L^{2}\left(0, T ; L_{n}^{2}\left(\Omega ; \mathbb{S}^{3}\right)\right)$ such that $\Xi_{i j}=0$ if $3 \in\{i, j\}$. By (5.10), the sequences $\left(\boldsymbol{u}_{\varepsilon} m_{\varepsilon}\right),\left(\frac{\partial \boldsymbol{u}_{\varepsilon}}{\partial t} m_{\varepsilon}\right)$ and $\left(\boldsymbol{e}_{x^{\prime}}\left(\boldsymbol{u}_{\varepsilon}^{\prime}\right) m_{\varepsilon}\right)$ are bounded in $L^{\infty}(0, T ; \mathcal{M}(\bar{\Omega}))$, therefore the convergences (5.15) also hold with respect to the weak* topology of $L^{\infty}(0, T ; \mathcal{M}(\bar{\Omega}))$. Let us fix $\boldsymbol{\Psi} \in C^{\infty}(\overline{\Omega \times(0, T)}$; $\mathbb{S}^{2}$ ). By integration by parts, we have

$$
\sum_{\alpha, \beta=1}^{2} \int_{\Omega \times(0, T)}\left(\boldsymbol{e}_{x^{\prime}}\left(\boldsymbol{u}_{\varepsilon}^{\prime}\right)\right)_{\alpha \beta} \Psi_{\alpha \beta}(x, t) d m_{\varepsilon} d t=-\int_{\Omega \times(0, T)}\left(u_{\varepsilon 1} \boldsymbol{e}_{1}+u_{\varepsilon 2} \boldsymbol{e}_{2}\right) \cdot \operatorname{div}_{x^{\prime}} \boldsymbol{\Psi}(x, t) d m_{\varepsilon} d t
$$

By passing to the limit as $\varepsilon \rightarrow 0$, taking (5.15) into account, we obtain 


$$
\sum_{\alpha, \beta=1}^{2} \int_{\Omega \times(0, T)} \Xi_{\alpha \beta} \Psi_{\alpha \beta} n d x d t=-\int_{\Omega \times(0, T)}\left(v_{1} \boldsymbol{e}_{1}+v_{2} \boldsymbol{e}_{2}\right) \cdot \operatorname{div}_{x^{\prime}} \mathbf{\Psi} n d x d t
$$

By making $\boldsymbol{\Psi}$ vary in $\mathcal{D}\left(\Omega \times(0, T) ; \mathbb{S}^{2}\right)$, we deduce that $n \boldsymbol{e}_{x^{\prime}}\left(\boldsymbol{v}^{\prime}\right)\left(=\boldsymbol{e}_{x^{\prime}}\left(n \boldsymbol{v}^{\prime}\right)\right)=n \boldsymbol{\Xi}$ in the sense of distributions on $\Omega \times(0, T)$, and then infer from Korn inequality in $H^{1}\left(\Omega^{\prime}\right)$ that $n v_{1}, n v_{2} \in L^{\infty}\left(0, T ; L^{2}\left(0, L ; H^{1}\left(\Omega^{\prime}\right)\right)\right)$, that is $v_{1}, v_{2} \in L^{\infty}\left(0, T ; L_{n}^{2}\left(0, L ; H^{1}\left(\Omega^{\prime}\right)\right)\right)$. By integrating (5.16) by parts for $\boldsymbol{\Psi} \in C^{\infty}\left(\overline{\Omega \times(0, T)} ; \mathbb{S}^{2}\right)$, we get $\int_{0}^{T} \int_{0}^{L} \int_{\partial \Omega^{\prime}} n \boldsymbol{v}^{\prime} \cdot \boldsymbol{\Psi} \boldsymbol{\nu}$ $d \mathcal{H}^{1} d x_{3} d t=0$ and infer from the arbitrariness of $\boldsymbol{\Psi}$ that $v_{1}, v_{2} \in L^{\infty}\left(0, T ; L_{n}^{2}(0, L\right.$; $\left.H_{0}^{1}\left(\Omega^{\prime}\right)\right)$ ). We prove in a similar way that $\boldsymbol{v} \in W^{1, \infty}\left(0, T ; L_{n}^{2}\left(\Omega ; \mathbb{R}^{3}\right)\right)$ and $\boldsymbol{\gamma}=\frac{\partial \boldsymbol{v}}{\partial t}$. Assertion (5.11) is proved. Assertion (5.12) is a consequence of the following inequalities (holding for $\alpha \in\{1,2\}$ ), deduced from (4.17), (5.1), (5.15)

$$
\begin{aligned}
& \int_{\Omega \times(0, T)}\left|v_{\alpha}\right|^{2} n d x d t \leq \liminf _{\varepsilon \rightarrow 0} \int_{\Omega \times(0, T)}\left|u_{\varepsilon \alpha}\right|^{2} d m_{\varepsilon}(x) d t \leq \liminf _{\varepsilon \rightarrow 0} \int_{\Omega \times(0, T)}\left|\boldsymbol{e}\left(\boldsymbol{u}_{\varepsilon}\right)\right|^{2} d m_{\varepsilon}(x) d t, \\
& \int_{\Omega \times(0, T)}|\boldsymbol{v}|^{2} n d x d t \leq \liminf _{\varepsilon \rightarrow 0} \int_{\Omega \times(0, T)}\left|\boldsymbol{u}_{\varepsilon}\right|^{2} d m_{\varepsilon}(x) d t \leq \liminf _{\varepsilon \rightarrow 0} \int_{\Omega \times(0, T)}\left|\frac{1}{r_{\varepsilon}} \boldsymbol{e}\left(\boldsymbol{u}_{\varepsilon}\right)\right|^{2} d m_{\varepsilon}(x) d t .
\end{aligned}
$$

It remains to show that under (5.13) or $(5.14), n \boldsymbol{u}=n \boldsymbol{v}$. To that aim, we set

$$
\hat{\boldsymbol{v}}_{\varepsilon}(x, t):=\sum_{j \in J_{\varepsilon}} \boldsymbol{u}_{\varepsilon}\left(x_{1}, x_{2}, \omega_{\varepsilon}^{j}, t\right) \mathbb{1}_{\left(\omega_{\varepsilon}^{j}-\frac{r_{\varepsilon}}{2}, \omega_{\varepsilon}^{j}+\frac{r_{\varepsilon}}{2}\right]}\left(x_{3}\right) .
$$

By Fubini's Theorem, Jensen's inequality and Korn's inequality in $H_{0}^{1}\left(\Omega ; \mathbb{R}^{3}\right)$, we have

$$
\begin{gathered}
\int\left|\boldsymbol{u}_{\varepsilon}-\hat{\boldsymbol{v}}_{\varepsilon}\right|^{2}(\tau) d m_{\varepsilon}=\frac{\varepsilon}{r_{\varepsilon}} \sum_{j \in J_{\varepsilon}} \int_{\Omega^{\prime}} d x^{\prime} \int_{\omega_{\varepsilon}^{j}-\frac{r_{\varepsilon}}{2}}^{\omega_{\varepsilon}^{j}+\frac{r_{\varepsilon}}{2}}\left|\boldsymbol{u}_{\varepsilon}\left(x^{\prime}, x_{3}, \tau\right)-\boldsymbol{u}_{\varepsilon}\left(x^{\prime}, \omega_{\varepsilon}^{j}, \tau\right)\right|^{2} d x_{3} \\
\leq \frac{\varepsilon}{r_{\varepsilon}} \sum_{j \in J_{\varepsilon}} \int_{\Omega^{\prime}} d x^{\prime} \int_{\omega_{\varepsilon}^{j}-\frac{r_{\varepsilon}}{2}}^{\omega_{\varepsilon}^{j}+\frac{r_{\varepsilon}}{2}}\left(\int_{\omega_{\varepsilon}^{j}-\frac{r_{\varepsilon}}{2}}^{\omega_{\varepsilon}^{j}+\frac{r_{\varepsilon}}{2}}\left|\frac{\partial \boldsymbol{u}_{\varepsilon}}{\partial x_{3}}\right|\left(x^{\prime}, s_{3}, \tau\right) d s_{3}\right)^{2} d x_{3} \\
\leq \varepsilon r_{\varepsilon} \int_{\Omega}\left|\nabla \boldsymbol{u}_{\varepsilon}\right|^{2}(\tau) d x \leq C \varepsilon r_{\varepsilon} \int_{\Omega}\left|\boldsymbol{e}\left(\boldsymbol{u}_{\varepsilon}\right)\right|^{2}(\tau) d x .
\end{gathered}
$$

Therefore, under (5.13) or (5.14), $\lim _{\varepsilon \rightarrow 0} \int\left|\boldsymbol{u}_{\varepsilon}-\hat{\boldsymbol{v}}_{\varepsilon}\right|^{2}(\tau) d m_{\varepsilon}=0$. Hence, by (5.15),

$$
\hat{\boldsymbol{v}}_{\varepsilon} m_{\varepsilon} \stackrel{\star}{\rightarrow} n \boldsymbol{v} \quad \text { weakly* in } L^{\infty}\left(0, T ; \mathcal{M}\left(\bar{\Omega} ; \mathbb{R}^{3}\right)\right) .
$$

We define (see $(3.1,3.15))$

$$
\overline{\boldsymbol{v}}_{\varepsilon}(x, t):=\sum_{i \in Z_{\varepsilon}}\left(\sum_{\left\{\omega_{\varepsilon}^{j} \in \omega_{\varepsilon} \cap\left(\varepsilon i-\frac{\varepsilon}{2}, \varepsilon i+\frac{\varepsilon}{2}\right]\right\}} \boldsymbol{u}_{\varepsilon}\left(x^{\prime}, \omega_{\varepsilon}^{j}, t\right)\right) \mathbb{1}_{\left(\varepsilon i-\frac{\varepsilon}{2}, \varepsilon i+\frac{\varepsilon}{2}\right]}\left(x_{3}\right) .
$$

Noticing that by (3.2) and (3.15), 


$$
\omega_{\varepsilon} \subset \bigcup_{i \in Z_{\varepsilon}}\left(\varepsilon i-\frac{\varepsilon}{2}, \varepsilon i+\frac{\varepsilon}{2}\right],
$$

we infer from (3.39) and (5.17) that

$$
\begin{aligned}
\int_{\Omega}\left|\overline{\boldsymbol{v}}_{\varepsilon}\right|^{2}(\tau) d x & \leq C \sum_{i \in Z_{\varepsilon}} \sum_{\left\{\omega_{\varepsilon}^{j} \in \omega_{\varepsilon} \cap\left(\varepsilon i-\frac{\varepsilon}{2}, \varepsilon i+\frac{\varepsilon}{2}\right]\right\}} \varepsilon \int_{\Omega^{\prime}}\left|\boldsymbol{u}_{\varepsilon}\left(x^{\prime}, \omega_{\varepsilon}^{j}, \tau\right)\right|^{2} d x^{\prime} \\
& =C \frac{\varepsilon}{r_{\varepsilon}} \sum_{j \in J_{\varepsilon}} r_{\varepsilon} \int_{\Omega^{\prime}}\left|\boldsymbol{u}_{\varepsilon}\left(x^{\prime}, \omega_{\varepsilon}^{j}, \tau\right)\right|^{2} d x^{\prime}=C \int\left|\hat{\boldsymbol{v}}_{\varepsilon}\right|^{2}(\tau) d m_{\varepsilon} .
\end{aligned}
$$

Therefore, by (5.10) and (5.18), under (5.13) or (5.14), the sequence $\left(\overline{\boldsymbol{v}}_{\varepsilon}\right)$ is bounded in $L^{\infty}\left(0, T ; L^{2}\left(\Omega ; \mathbb{R}^{3}\right)\right)$. Thus the following convergence holds, up to a subsequence

$$
\overline{\boldsymbol{v}}_{\varepsilon} \stackrel{\star}{\rightarrow} \boldsymbol{w} \quad \text { weakly* in } L^{\infty}\left(0, T ; L^{2}\left(\Omega ; \mathbb{R}^{3}\right)\right) .
$$

To identify $\boldsymbol{w}$, we fix $\boldsymbol{\varphi} \in \mathcal{D}\left(\Omega \times(0, T) ; \mathbb{R}^{3}\right)$ and set $\breve{\varphi}_{\varepsilon}(x, t):=\sum_{i \in Z_{\varepsilon}} \varphi\left(x^{\prime}, \varepsilon i, t\right)$ $\mathbb{1}_{\left(\varepsilon i-\frac{\varepsilon}{2}, \varepsilon i+\frac{\varepsilon}{2}\right]}\left(x_{3}\right)$. Noticing that $\left|\breve{\varphi}_{\varepsilon}-\varphi\right|_{L^{\infty}\left(\Omega \times(0, T) ; \mathbb{R}^{3}\right)} \leq C \varepsilon$, we infer

$$
\int_{\Omega \times(0, T)} \boldsymbol{w} \cdot \boldsymbol{\varphi} d x d t=\lim _{\varepsilon \rightarrow 0} \int_{\Omega \times(0, T)} \overline{\boldsymbol{v}}_{\varepsilon} \cdot \breve{\boldsymbol{\varphi}}_{\varepsilon} d x d t .
$$

On the other hand, by (3.39), (5.17) and (5.20), we have

$$
\begin{aligned}
& \int_{\Omega} \overline{\boldsymbol{v}}_{\varepsilon} \cdot \breve{\boldsymbol{\varphi}}_{\varepsilon}(t) d x=\sum_{i \in Z_{\varepsilon}} \sum_{\left\{\omega_{\varepsilon}^{j} \in \omega_{\varepsilon} \cap\left(\varepsilon i-\frac{\varepsilon}{2}, \varepsilon i+\frac{\varepsilon}{2}\right]\right\}} \int_{\Omega^{\prime} \times\left(\varepsilon i-\frac{\varepsilon}{2}, \varepsilon i+\frac{\varepsilon}{2}\right]} \boldsymbol{u}_{\varepsilon}\left(x^{\prime}, \omega^{j}, t\right) \cdot \boldsymbol{\varphi}\left(x^{\prime}, \varepsilon i, t\right) d x \\
& =\sum_{i \in Z_{\varepsilon}} \sum_{\left\{\omega_{\varepsilon}^{j} \in \omega_{\varepsilon} \cap\left(\varepsilon i-\frac{\varepsilon}{2}, \varepsilon i+\frac{\varepsilon}{2}\right]\right\}} \int_{\Omega^{\prime} \times\left(\omega_{\varepsilon}^{j}+\frac{r_{\varepsilon}}{2}, \omega_{\varepsilon}^{j}+\frac{r_{\varepsilon}}{2}\right)} \hat{\boldsymbol{\varphi}}_{\frac{1}{2}}\left(x^{\prime}, \varepsilon i, t\right) d m_{\varepsilon} .
\end{aligned}
$$

For all $i \in Z_{\varepsilon}$ and all $\omega_{\varepsilon}^{j} \in \omega_{\varepsilon} \cap\left(\varepsilon i-\frac{\varepsilon}{2}, \varepsilon i+\frac{\varepsilon}{2}\right]$, we have $\left|\varphi\left(x_{1}, x_{2}, \varepsilon i, t\right)-\varphi(x, t)\right| \leq C \varepsilon$ in $\Omega^{\prime} \times\left(\omega_{\varepsilon}^{j}+\frac{r_{\varepsilon}}{2}, \omega_{\varepsilon}^{j}+\frac{r_{\varepsilon}}{2}\right) \times(0, T)$. Taking (5.21) into account, we deduce

$$
\begin{array}{r}
\left|\sum_{i \in Z_{\varepsilon}} \sum_{\left\{\omega_{\varepsilon}^{j} \in \omega_{\varepsilon} \cap\left(\varepsilon i-\frac{\varepsilon}{2}, \varepsilon i+\frac{\varepsilon}{2}\right]\right\}} \int_{\Omega^{\prime} \times\left(\omega_{\varepsilon}^{j}+\frac{r_{\varepsilon}}{2}, \omega_{\varepsilon}^{j}+\frac{r_{\varepsilon}}{2}\right)} \hat{\varphi}_{\left(x^{\prime}, \varepsilon i, t\right) d m_{\varepsilon}}-\int \hat{\boldsymbol{v}}_{\varepsilon}(t) \cdot \boldsymbol{\varphi}(t) d m_{\varepsilon}\right| \\
\leq C \varepsilon \int\left|\hat{\boldsymbol{v}}_{\varepsilon}\right|(t) d m_{\varepsilon} .
\end{array}
$$

Therefore, by (5.19) and (5.25), the following holds

$$
\lim _{\varepsilon \rightarrow 0} \int_{\Omega \times(0, T)} \overline{\boldsymbol{v}}_{\varepsilon} \cdot \breve{\varphi}_{\varepsilon} d x d t=\lim _{\varepsilon \rightarrow 0} \int_{\Omega \times(0, T)} \hat{\boldsymbol{v}}_{\varepsilon} \cdot \boldsymbol{\varphi} d m_{\varepsilon} d t=\int_{\Omega \times(0, T)} n \boldsymbol{v} \cdot \boldsymbol{\varphi} d x d t
$$


Combining (5.24) and (5.26) we deduce, by the arbitrary choice of $\boldsymbol{\varphi}$,

$$
\boldsymbol{w}=n \boldsymbol{v} .
$$

On the other hand, by (3.14), (5.20), (5.21), Jensen's inequality and Korn's inequality in $H_{0}^{1}(\Omega)$, we have

$$
\begin{aligned}
& \int_{\Omega}\left|n_{\varepsilon} \boldsymbol{u}_{\varepsilon}-\overline{\boldsymbol{v}}_{\varepsilon}\right|^{2}(\tau) d x \\
& \quad=\sum_{i \in Z_{\varepsilon}} \int_{\Omega^{\prime} \times\left(\varepsilon i-\frac{\varepsilon}{2}, \varepsilon i+\frac{\varepsilon}{2}\right]}\left|\sum_{\left\{\omega_{\varepsilon}^{j} \in \omega_{\varepsilon} \cap\left(\varepsilon i-\frac{\varepsilon}{2}, \varepsilon i+\frac{\varepsilon}{2}\right]\right\}} \boldsymbol{u}_{\varepsilon}-\boldsymbol{u}_{\varepsilon}\left(x^{\prime}, \omega_{\varepsilon}^{j}, \tau\right)\right|^{2} d x \\
& \quad \leq C \sum_{i \in Z_{\varepsilon}} \sum_{\left\{\omega_{\varepsilon}^{j} \in \omega_{\varepsilon} \cap\left(\varepsilon i-\frac{\varepsilon}{2}, \varepsilon i+\frac{\varepsilon}{2}\right]\right\}} \int_{\Omega^{\prime} \times\left(\varepsilon i-\frac{\varepsilon}{2}, \varepsilon i+\frac{\varepsilon}{2}\right]}\left|\boldsymbol{u}_{\varepsilon}-\boldsymbol{u}_{\varepsilon}\left(x^{\prime}, \omega_{\varepsilon}^{j}, \tau\right)\right|^{2} d x \\
& \quad \leq C \varepsilon^{2} \sum_{i \in Z_{\varepsilon}} n_{\varepsilon}(\varepsilon i) \int_{\Omega^{\prime} \times\left(\varepsilon i-\frac{\varepsilon}{2}, \varepsilon i+\frac{\varepsilon}{2}\right]}\left|\frac{\partial \boldsymbol{u}_{\varepsilon}}{\partial x_{3}}\right|^{2}(\tau) d x \leq C \varepsilon^{2} \int_{\Omega}\left|\boldsymbol{e}\left(\boldsymbol{u}_{\varepsilon}\right)\right|^{2}(\tau) d x .
\end{aligned}
$$

We infer that, under either (5.13) or (5.14), the sequence $\left(\int_{\Omega \times(0, T)}\left|n_{\varepsilon} \boldsymbol{u}_{\varepsilon}-\overline{\boldsymbol{v}}_{\varepsilon}\right|^{2}(\tau)\right.$ $d x d t)$ converges to 0 . Therefore, by (5.23) and (5.27),

$$
n_{\varepsilon} \boldsymbol{u}_{\varepsilon} \stackrel{\star}{*} n \boldsymbol{v} \quad \text { weakly* in } L^{\infty}\left(0, T ; L^{2}\left(\Omega ; \mathbb{R}^{3}\right)\right) .
$$

Under (5.14), it easily follows from the strong convergence of $\left(\boldsymbol{u}_{\varepsilon}\right)$ to $\boldsymbol{u}$ in $L^{2}\left(0, T ; L^{2}(\Omega\right.$; $\left.\mathbb{R}^{3}\right)$ ) and the weak* convergence of $\left(n_{\varepsilon}\right)$ to $n$ in $L^{\infty}(\Omega)$ (see (3.16)), that $\left(n_{\varepsilon} \boldsymbol{u}_{\varepsilon}\right)$ weakly converges to $n \boldsymbol{u}$ in $L^{2}\left(0, T ; L^{2}\left(\Omega ; \mathbb{R}^{3}\right)\right)$, therefore $n \boldsymbol{u}=n \boldsymbol{v}$. The same conclusion holds under (5.13), because $\left(\boldsymbol{u}_{\varepsilon}\right)$ weakly* converges to $\boldsymbol{u}$ in $L^{\infty}\left(0, T ; L^{2}\left(\Omega ; \mathbb{R}^{3}\right)\right)$ and $\left(n_{\varepsilon}\right)$ is bounded in $L^{\infty}(\Omega)$ and strongly converges to $n$ in $L^{2}(\Omega)$. The proof of Lemma 5.3 is concluded.

LEMMA 5.4. Let $\left(\boldsymbol{u}_{\varepsilon}\right)$ be a sequence in $L^{\infty}\left(0, T ; H_{0}^{1}\left(\Omega ; \mathbb{R}^{3}\right)\right)$ satisfying

$$
\sup _{\varepsilon>0, \tau \in(0, T)} \int\left|\frac{\partial \boldsymbol{u}_{\varepsilon}}{\partial t}\right|^{2}+\left|\frac{1}{r_{\varepsilon}} \boldsymbol{e}\left(\boldsymbol{u}_{\varepsilon}\right)\right|^{2}(\tau) d m_{\varepsilon}<+\infty .
$$

Then, up to a subsequence, the convergences (5.11) take place. Moreover,

$$
v_{3} \in L^{\infty}\left(0, T ; L_{n}^{2}\left(0, L ; H_{0}^{2}\left(\Omega^{\prime}\right)\right)\right) .
$$

Besides, up to a subsequence, the following convergences hold (see (4.10)):

$$
\begin{aligned}
& \frac{u_{\varepsilon \alpha}}{r_{\varepsilon}} \stackrel{m_{\varepsilon}}{\xi_{\alpha}}(x, t)-\frac{\partial v_{3}}{\partial x_{\alpha}}(x, t) y_{3} \quad(\alpha \in\{1,2\}), \\
& \left(\frac{1}{r_{\varepsilon}} \boldsymbol{e}\left(\boldsymbol{u}_{\varepsilon}\right)\right)_{\alpha \beta} \stackrel{m_{\varepsilon}}{\longrightarrow} \frac{1}{2}\left(\frac{\partial \xi_{\alpha}}{\partial x_{\beta}}+\frac{\partial \xi_{\beta}}{\partial x_{\alpha}}\right)(x, t)-\frac{\partial^{2} v_{3}}{\partial x_{\alpha} \partial x_{\beta}}(x, t) y_{3} \quad(\alpha, \beta \in\{1,2\}), \\
& \xi_{1}, \xi_{2} \in L^{\infty}\left(0, T ; L_{n}^{2}\left(0, L ; H_{0}^{1}\left(\Omega^{\prime}\right)\right)\right) .
\end{aligned}
$$


Proof. By (5.1) and (5.30), we have

$$
\sup _{\varepsilon>0, \tau \in(0, T)} \int\left(\left|\frac{u_{\varepsilon 1}}{r_{\varepsilon}}\right|^{2}+\left|\frac{u_{\varepsilon 2}}{r_{\varepsilon}}\right|^{2}+\left|u_{\varepsilon 3}\right|^{2}\right) d m_{\varepsilon}<+\infty .
$$

By (5.30) and (5.33), assumption (5.10) of Lemma 5.3 is verified, hence, up to a subsequence, the convergences (5.11) take place. By Lemma 4.2, (5.30) and (5.33), there exist $\boldsymbol{v} \in L^{\infty}\left(0, T ; L_{n}^{2}\left(\Omega ; \mathbb{R}^{3}\right)\right), \zeta_{01}, \zeta_{02}, \zeta_{03} \in L^{\infty}\left(0, T ; L_{n}^{2}(\Omega \times I)\right)$, and $\boldsymbol{\Xi}^{b} \in$ $L^{\infty}\left(0, T ; L_{n}^{2}\left(\Omega \times I ; \mathbb{S}^{3}\right)\right)$, such that (see $\left.(2.1)\right)$

$$
\begin{aligned}
& \boldsymbol{u} m_{\varepsilon} \stackrel{\star}{\rightarrow} n \boldsymbol{v} \quad \text { weakly* in } L^{\infty}\left(0, T ; \mathcal{M}\left(\bar{\Omega} ; \mathbb{R}^{3}\right)\right) ; \quad n v_{1}=n v_{2}=0 ; \\
& u_{\varepsilon 3} \stackrel{m_{\varepsilon}}{\zeta_{03} ;} \quad \frac{u_{\varepsilon \alpha}}{r_{\varepsilon}} \stackrel{m_{\varepsilon}}{=} \zeta_{0 \alpha} \quad(\alpha \in\{1,2\}) ; \quad \frac{1}{r_{\varepsilon}} \boldsymbol{e}_{x^{\prime}}\left(\boldsymbol{u}_{\varepsilon}\right) \stackrel{m_{\varepsilon}}{\boldsymbol{\Xi}^{b}} .
\end{aligned}
$$

We will use the following assertion, which will be proved later,

$$
n(x) \zeta_{03}\left(x, t, y_{3}\right)=n(x) v_{3}(x, t) \quad \text { a.e. in } \Omega \times(0, T) \times I
$$

Then, we fix a matrix field $\boldsymbol{\Psi}$ satisfying

$$
\Psi \in C^{\infty}\left(\overline{\Omega \times(0, T)} ; \mathcal{D}\left(I ; \mathbb{S}^{3}\right)\right), \quad \Psi_{33}=0 .
$$

Noticing that $x \rightarrow \Psi\left(x, t, \frac{y_{\varepsilon}\left(x_{3}\right)}{r_{\varepsilon}}\right)$ vanishes on the complement of the support of $m_{\varepsilon}$, by integration by parts we get

$$
\begin{aligned}
& \int_{\Omega \times(0, T)} \boldsymbol{e}\left(\boldsymbol{u}_{\varepsilon}\right): \Psi\left(x, t, \frac{y_{\varepsilon}\left(x_{3}\right)}{r_{\varepsilon}}\right) d m_{\varepsilon} d t=-\int_{\Omega \times(0, T)} \boldsymbol{u}_{\varepsilon} \cdot \operatorname{div}_{x} \boldsymbol{\Psi}\left(x, t, \frac{y_{\varepsilon}\left(x_{3}\right)}{r_{\varepsilon}}\right) d m_{\varepsilon} d t \\
& -\sum_{\alpha=1}^{2} \int_{\Omega \times(0, T)} \frac{u_{\varepsilon \alpha}}{r_{\varepsilon}} \frac{\partial \Psi_{\alpha 3}}{\partial y_{3}}\left(x, t, \frac{y_{\varepsilon}\left(x_{3}\right)}{r_{\varepsilon}}\right) d m_{\varepsilon} d t
\end{aligned}
$$

By passing to the limit as $\varepsilon \rightarrow 0$ in (5.37), taking (5.30), (5.34) and (5.35) into account, we infer

$$
0=-\int_{\Omega \times(0, T) \times I} v_{3}\left(\operatorname{div}_{x} \boldsymbol{\Psi}\right)_{3} n d x d t d y_{3}-\sum_{\alpha=1}^{2} \int_{\Omega \times(0, T) \times I} \zeta_{0 \alpha} \frac{\partial \Psi_{\alpha 3}}{\partial y_{3}} n d x d t d y_{3}
$$

Fixing $\alpha \in\{1,2\}, \varphi \in C^{\infty}(\overline{\Omega \times(0, T)}), \psi \in \mathcal{D}(I)$, and selecting in (5.38) a field of the form $\boldsymbol{\Psi}\left(x, t, y_{3}\right):=\varphi(x, t) \psi\left(y_{3}\right)\left(\boldsymbol{e}_{\alpha} \otimes \boldsymbol{e}_{3}+\boldsymbol{e}_{3} \otimes \boldsymbol{e}_{\alpha}\right)$, we get

$$
\begin{aligned}
0=-\int_{\Omega \times(0, T)} v_{3}(x, t) & \frac{\partial \varphi}{\partial x_{\alpha}}(x, t) n d x d t\left(\int_{I} \psi\left(y_{3}\right) d y_{3}\right) \\
& -\int_{\Omega \times(0, T)}\left(\int_{I} \zeta_{0 \alpha}\left(x, t, y_{3}\right) \frac{\partial \psi}{\partial y_{3}}\left(y_{3}\right) d y_{3}\right) \varphi(x, t) n d x d t .
\end{aligned}
$$


Choosing $\psi$ such that $\left(\int_{I} \psi\left(y_{3}\right) d y_{3}\right) \neq 0$, and making $\varphi$ vary in $C^{\infty}(\overline{\Omega \times(0, T)})$, we deduce that

$$
v_{3} \in L^{\infty}\left(0, T ; L_{n}^{2}\left(0, L ; H_{0}^{1}\left(\Omega^{\prime}\right)\right)\right)
$$

then, by integration by parts with respect to $x_{\alpha}$, infer

$$
0=\int_{\Omega \times(0, T) \times I} \varphi(x, t) \psi\left(y_{3}\right) \frac{\partial v_{3}}{\partial x_{\alpha}}(x, t) n d x d t d y_{3}-\int_{\Omega \times(0, T) \times I} \zeta_{0 \alpha}\left(x, t, y_{3}\right) \frac{\partial \psi}{\partial y_{3}}\left(y_{3}\right) \varphi(x, t) n d x d t d y_{3} .
$$

We deduce, from the arbitrary choice of $\varphi$ and $\psi$, that

$$
\zeta_{0 \alpha} \in L^{\infty}\left(0, T ; L_{n}^{2}\left(\Omega ; H^{1}(I)\right)\right) ; n \frac{\partial \zeta_{0 \alpha}}{\partial y_{3}}\left(x, t, y_{3}\right)=-n \frac{\partial v_{3}}{\partial x_{\alpha}}(x, t) \text { in } \Omega \times(0, T) \times I,
$$

and then that

$$
n \zeta_{0 \alpha}\left(x, t, y_{3}\right)=n \xi_{\alpha}(x, t)-n \frac{\partial v_{3}}{\partial x_{\alpha}}(x, t) y_{3} \quad \text { in } \Omega \times(0, T) \times I,
$$

for some suitable $\xi_{\alpha} \in L^{\infty}\left(0, T ; L_{n}^{2}(\Omega)\right)$. Next, we choose a matrix field $\boldsymbol{\Psi}$ satisfying (5.36) and $\Psi_{3 k}=0 \forall k \in\{1,2,3\}$. By multiplying (5.37) by $\frac{1}{r_{\varepsilon}}$, we get

$$
\begin{aligned}
\sum_{\alpha, \beta=1}^{2} \int_{\Omega \times(0, T)} \frac{e_{\alpha \beta}\left(\boldsymbol{u}_{\varepsilon}\right)}{r_{\varepsilon}} \Psi_{\alpha \beta} & \left(x, t, \frac{y_{\varepsilon}\left(x_{3}\right)}{r_{\varepsilon}}\right) d m_{\varepsilon} d t= \\
& -\sum_{\alpha, \beta=1}^{2} \int_{\Omega \times(0, T)} \frac{u_{\varepsilon \alpha}}{r_{\varepsilon}} \frac{\Psi_{\alpha \beta}}{\partial x_{\beta}}\left(x, t, \frac{y_{\varepsilon}\left(x_{3}\right)}{r_{\varepsilon}}\right) d m_{\varepsilon} d t .
\end{aligned}
$$

By passing to the limit as $\varepsilon \rightarrow 0$, thanks to (5.34) and (5.40), we find

$$
\begin{aligned}
& \sum_{\alpha, \beta=1}^{2} \int_{\Omega \times(0, T) \times I} \Xi_{\alpha \beta}^{b} \Psi_{\alpha \beta} n d x d t d y \\
& \quad=-\sum_{\alpha, \beta=1}^{2} \int_{\Omega \times(0, T) \times I}\left(\xi_{\alpha}(x, t)-\frac{\partial v_{3}}{\partial x_{\alpha}}(x, t) y_{3}\right) \frac{\partial \Psi_{\alpha \beta}}{\partial x_{\beta}}\left(x, t, y_{3}\right) n d x d t d y_{3} .
\end{aligned}
$$

By the arbitrary choice of the functions $\Psi_{\alpha \beta}\left(=\Psi_{\beta \alpha}\right)$ in $C^{\infty}(\overline{\Omega \times(0, T)} ; \mathcal{D}(I))$ and (5.39), we deduce that for $\alpha, \beta \in\{1,2\}$, the following holds

$$
\begin{aligned}
& \xi_{\alpha} \in L^{\infty}\left(0, T ; L_{n}^{2}\left(0, L ; H_{0}^{1}\left(\Omega^{\prime}\right)\right)\right), \quad v_{3} \in L^{\infty}\left(0, T ; L_{n}^{2}\left(0, L ; H_{0}^{2}\left(\Omega^{\prime}\right)\right)\right), \\
& n \Xi_{\alpha \beta}^{b}\left(x, t, y_{3}\right)=\frac{1}{2} n\left(\frac{\partial \xi_{\alpha}}{\partial x_{\beta}}+\frac{\partial \xi_{\beta}}{\partial x_{\alpha}}\right)(x, t)-n \frac{\partial^{2} v_{3}}{\partial x_{\alpha} \partial x_{\beta}}(x, t) y_{3} \text { in } \Omega \times(0, T) \times I .
\end{aligned}
$$

The proof of Lemma 5.4 is concluded.

Proof of (5.35). By (5.17) and (5.30), we have 


$$
\begin{aligned}
& \int\left|u_{\varepsilon 3}-\hat{v}_{\varepsilon 3}\right|^{2}(\tau) d m_{\varepsilon}=\frac{\varepsilon}{r_{\varepsilon}} \sum_{j \in J_{\varepsilon}} \int_{\Omega^{\prime}} d x^{\prime} \int_{\omega_{\varepsilon}^{j}-\frac{r_{\varepsilon}}{2}}^{\omega_{\varepsilon}^{j}+\frac{r_{\varepsilon}}{2}}\left|u_{\varepsilon 3}(x, \tau)-u_{\varepsilon 3}\left(x^{\prime}, \omega_{\varepsilon}^{j}, \tau\right)\right|^{2} d x_{3} \\
& \quad \leq \varepsilon r_{\varepsilon} \sum_{j \in J_{\varepsilon}} \int_{\Omega^{\prime}} d x^{\prime} \int_{\omega_{\varepsilon}^{j}-\frac{r_{\varepsilon}}{2}}^{\omega_{\varepsilon}^{j}+\frac{r_{\varepsilon}}{2}}\left|\frac{\partial u_{\varepsilon 3}}{\partial x_{3}}\right|^{2}\left(x^{\prime}, x_{3}, \tau\right) d x_{3} \leq C r_{\varepsilon}^{2} \int\left|\boldsymbol{e}\left(\boldsymbol{u}_{\varepsilon}\right)\right|^{2}(\tau) d m_{\varepsilon} \leq C r_{\varepsilon}^{4} .
\end{aligned}
$$

We easily deduce from (5.34) and (5.41) that

$$
\hat{v}_{\varepsilon 3} m_{\varepsilon} \stackrel{\star}{\rightarrow} n v_{3} \quad \text { weakly* in } L^{\infty}(0, T ; \mathcal{M}(\bar{\Omega})) ; \quad \hat{v}_{\varepsilon 3} \stackrel{m_{\varepsilon}}{\longrightarrow} \zeta_{03} .
$$

Fixing $\psi \in \mathcal{D}(\Omega \times(0, T) \times I)$, we set (see (4.9))

$$
\widetilde{\psi}_{\varepsilon}\left(x, t, \frac{y_{\varepsilon}\left(x_{3}\right)}{r_{\varepsilon}}\right):=\sum_{j \in J_{\varepsilon}} \psi\left(x^{\prime}, \omega_{\varepsilon}^{j}, t, \frac{y_{\varepsilon}\left(x_{3}\right)}{r_{\varepsilon}}\right) \mathbb{1}_{\left(\omega_{\varepsilon}^{j}-\frac{r_{\varepsilon}}{2}, \omega_{\varepsilon}^{j}+\frac{r_{\varepsilon}}{2}\right)}\left(x_{3}\right) .
$$

We have

$$
\left|\widetilde{\psi}_{\varepsilon}\left(x, t, \frac{y_{\varepsilon}\left(x_{3}\right)}{r_{\varepsilon}}\right)-\psi\left(x, t, \frac{y_{\varepsilon}\left(x_{3}\right)}{r_{\varepsilon}}\right)\right| \leq C r_{\varepsilon} \quad \text { in } B_{\varepsilon} .
$$

By making the change of variables $y=\frac{x_{3}-\omega_{\varepsilon}^{j}}{r_{\varepsilon}}$, we get $\int_{\omega_{\varepsilon}^{j}-\frac{r_{\varepsilon}}{2}}^{\omega_{j}^{j}+\frac{r_{\varepsilon}}{2}} \psi\left(x^{\prime}, \omega_{\varepsilon}^{j}, t, \frac{y_{\varepsilon}\left(x_{3}\right)}{r_{\varepsilon}}\right) d x_{3}=$ $r_{\varepsilon} \int_{I} \psi\left(x^{\prime}, \omega_{\varepsilon}^{j}, t, y_{3}\right) d y_{3}$. We infer

$$
\begin{aligned}
& \int_{\Omega \times(0, T)} \hat{v}_{\varepsilon 3} \widetilde{\psi}_{\varepsilon}\left(x, t, \frac{y_{\varepsilon}\left(x_{3}\right)}{r_{\varepsilon}}\right) d m_{\varepsilon} d t \\
& =\frac{\varepsilon}{r_{\varepsilon}} \sum_{j \in J_{\varepsilon}} \int_{\Omega^{\prime} \times(0, T)} d x^{\prime} d t \int_{\omega_{\varepsilon}^{j}-\frac{r_{\varepsilon}}{2}}^{\omega_{\varepsilon}^{j}+\frac{r_{\varepsilon}}{2}} u_{\varepsilon 3}\left(x^{\prime}, \omega_{\varepsilon}^{j}, t\right) \psi\left(x^{\prime}, \omega_{\varepsilon}^{j}, t, \frac{y_{\varepsilon}\left(x_{3}\right)}{r_{\varepsilon}}\right) d x_{3} \\
& =\frac{\varepsilon}{r_{\varepsilon}} \sum_{j \in J_{\varepsilon}} \int_{\Omega^{\prime} \times(0, T)} r_{\varepsilon} u_{\varepsilon 3}\left(x^{\prime}, \omega_{\varepsilon}^{j}, t\right)\left(\int_{I} \psi\left(x^{\prime}, \omega_{\varepsilon}^{j}, t, y_{3}\right) d y_{3}\right) d x^{\prime} d t \\
& =\frac{\varepsilon}{r_{\varepsilon}} \sum_{j \in J_{\varepsilon}} \int_{\Omega^{\prime} \times(0, T)} d x^{\prime} d t \int_{\omega_{\varepsilon}^{j}-\frac{r_{\varepsilon}}{2}}^{\omega_{\varepsilon}^{j}+\frac{r_{\varepsilon}}{2}} u_{\varepsilon 3}\left(x^{\prime}, \omega_{\varepsilon}^{j}, t\right)\left(\int_{I} \psi\left(x^{\prime}, \omega_{\varepsilon}^{j}, t, y_{3}\right) d y_{3}\right) d x_{3} \\
& =\int_{\Omega \times(0, T)} \hat{v}_{\varepsilon 3}(x, t) \hat{\psi}_{\varepsilon}(x, t) d m_{\varepsilon} d t
\end{aligned}
$$

where $\widehat{\psi}_{\varepsilon}(x, t):=\sum_{j \in J_{\varepsilon}}\left(\int_{I} \psi\left(x^{\prime}, \omega_{\varepsilon}^{j}, t, y_{3}\right) d y_{3}\right) \mathbb{1}_{\left(\omega_{\varepsilon}^{j}-\frac{r_{\varepsilon}}{2}, \omega_{\varepsilon}^{j}+\frac{r_{\varepsilon}}{2}\right)}\left(x_{3}\right)$. Noticing that $\left|\widehat{\psi}_{\varepsilon}(x, t)-\left(\int_{I} \psi\left(x, t, y_{3}\right) d y_{3}\right)\right| \leq C r_{\varepsilon}$ in $B_{\varepsilon}$, we deduce successively from (5.42), (5.45), (5.44), and again (5.42) that 


$$
\begin{aligned}
& \int_{\Omega \times(0, T) \times I} v_{3}(x, t)\left(\int_{I} \psi\left(x, t, y_{3}\right) d y_{3}\right) n d x d t=\lim _{\varepsilon \rightarrow 0} \int_{\Omega \times(0, T)} \hat{v}_{\varepsilon 3}(x, t)\left(\int_{I} \psi\left(x, t, y_{3}\right) d y_{3}\right) d m_{\varepsilon} d t \\
& =\lim _{\varepsilon \rightarrow 0} \int_{\Omega \times(0, T)} \hat{v}_{\varepsilon 3}(x, t) \widehat{\psi}_{\varepsilon}(x, t) d m_{\varepsilon} d t=\lim _{\varepsilon \rightarrow 0} \int_{\Omega \times(0, T)} \hat{v}_{\varepsilon 3}(x, t) \widetilde{\psi}_{\varepsilon}\left(x, t, \frac{y_{\varepsilon}\left(x_{3}\right)}{r_{\varepsilon}}\right) d m_{\varepsilon} d t \\
& =\lim _{\varepsilon \rightarrow 0} \int_{\Omega \times(0, T)} \hat{v}_{\varepsilon 3}(x, t) \psi\left(x, t, \frac{y_{\varepsilon}\left(x_{3}\right)}{r_{\varepsilon}}\right) d m_{\varepsilon} d t=\int_{\Omega \times(0, T) \times I}^{\zeta_{00}\left(x, t, y_{3}\right) \psi\left(x, t, y_{3}\right) n d x d t d y_{3}}
\end{aligned}
$$

By the arbitrary choice of $\psi$, assertion (5.35) is proved.

The next Lemma is specific to the periodic case. Given a sequence $\left(\boldsymbol{u}_{\varepsilon}\right)$ satisfying (5.10) and (5.47) (and possibly (5.30)), we establish some relations satisfied by its two-scale limit $\boldsymbol{u}_{0}$ and by the field $\boldsymbol{v}$ introduced in Lemma 5.3.

Lemma 5.5. Assume that $B_{\varepsilon}$ is the $\varepsilon$-periodic set defined by (3.35) and let $\left(\boldsymbol{u}_{\varepsilon}\right)$ be a sequence in $L^{\infty}\left(0, T ; H_{0}^{1}\left(\Omega ; \mathbb{R}^{3}\right)\right)$ satisfying (5.10) and

$$
\sup _{\tau \in[0, T], \varepsilon>0} \int_{\Omega}\left|\boldsymbol{u}_{\varepsilon}\right|^{2}+\left|\frac{\partial \boldsymbol{u}_{\varepsilon}}{\partial t}\right|^{2}+\varepsilon^{2}\left|\boldsymbol{e}\left(\boldsymbol{u}_{\varepsilon}\right)\right|^{2}(\tau) d x<+\infty, \quad \boldsymbol{u}_{\varepsilon}(0)=\boldsymbol{a}_{0} .
$$

Then, up to a subsequence, the convergences (5.11) take place with $n=1$. Moreover

$$
\begin{aligned}
& \boldsymbol{u}_{\varepsilon} \longrightarrow \boldsymbol{u}_{0} \text { and } \varepsilon \boldsymbol{e}\left(\boldsymbol{u}_{\varepsilon}\right) \longrightarrow \boldsymbol{e}_{y}\left(\boldsymbol{u}_{0}\right) \text { in accordance with (4.1), } \\
& \boldsymbol{u}_{0} \in L^{\infty}\left(0, T ; L^{2}\left(\Omega ; H_{\sharp}\left(Y ; \mathbb{R}^{3}\right)\right)\right) \cap W^{1, \infty}\left(0, T ; L^{2}\left(\Omega \times Y ; \mathbb{R}^{3}\right)\right), \\
& \frac{\partial \boldsymbol{u}_{\varepsilon}}{\partial t} \longrightarrow \frac{\partial \boldsymbol{u}_{0}}{\partial t}, \quad \boldsymbol{u}_{\varepsilon}(\tau) \longrightarrow \boldsymbol{u}_{0}(\tau) \forall \tau \in[0, T] .
\end{aligned}
$$

Furthermore,

(i) If $\vartheta>0$, then

$$
\boldsymbol{u}_{0}(x, t, y)=\boldsymbol{v}(x, t) \quad \text { in } \Omega \times(0, T) \times B .
$$

(ii) If $\vartheta=0$ and if the estimate (5.30) is satisfied, then

$$
\boldsymbol{u}_{0}(x, t, y)=\boldsymbol{v}(x, t) \quad \text { on } \Omega \times(0, T) \times \Sigma .
$$

Proof. The convergences (5.11) are deduced from Lemma 5.3. Under (5.47), by Lemma 4.1 (ii), the sequence $\left(\boldsymbol{u}_{\varepsilon}\right)$ (resp. $\left(\varepsilon \boldsymbol{e}\left(\boldsymbol{u}_{\varepsilon}\right)\right)$ ) two-scale converges, up to a subsequence, to some $\boldsymbol{u}_{0} \in L^{\infty}\left(0, T ; L^{2}\left(\Omega \times Y ; \mathbb{R}^{3}\right)\right)$ (resp. $\boldsymbol{\Xi}^{m} \in L^{\infty}\left(0, T ; L^{2}\left(\Omega \times Y ; \mathbb{S}^{3}\right)\right)$ ). Choosing $\Psi \in \mathcal{D}\left(\Omega \times(0, T) ; C_{\sharp}^{\infty}\left(Y ; \mathbb{S}^{3}\right)\right)$ and passing to the limit as $\varepsilon \rightarrow 0$ in the equation

$$
\begin{aligned}
& \int_{\Omega \times(0, T)} \varepsilon \boldsymbol{e}\left(\boldsymbol{u}_{\varepsilon}\right): \boldsymbol{\Psi}\left(x, t, \frac{x}{\varepsilon}\right) d x d t= \\
& \quad-\varepsilon \int_{\Omega \times(0, T)} \boldsymbol{u}_{\varepsilon} \cdot \operatorname{div}_{x} \boldsymbol{\Psi}\left(x, t, \frac{x}{\varepsilon}\right) d x d t-\int_{\Omega \times(0, T)} \boldsymbol{u}_{\varepsilon} \cdot \operatorname{div}_{y} \boldsymbol{\Psi}\left(x, t, \frac{x}{\varepsilon}\right) d x d t
\end{aligned}
$$


we infer $\int_{\Omega \times(0, T) \times Y} \boldsymbol{\Xi}^{m}: \boldsymbol{\Psi} d x d t d y=-\int_{\Omega \times(0, T) \times Y} \boldsymbol{u}_{0} \cdot \operatorname{div}_{y} \boldsymbol{\Psi} d x d t d y$ and deduce, by the arbitrary choice of $\boldsymbol{\Psi}$, that $\boldsymbol{u}_{0} \in L^{\infty}\left(0, T ; L^{2}\left(\Omega ; H_{\sharp}^{1}\left(Y ; \mathbb{R}^{3}\right)\right)\right)$ and $\boldsymbol{e}_{y}\left(\boldsymbol{u}_{0}\right)=\mathbf{\Xi}^{m}$. By Lemma 4.1 (ii), $\boldsymbol{u}_{0} \in W^{1, \infty}\left(0, T ; L^{2}\left(\Omega \times Y ; \mathbb{R}^{3}\right)\right)$ and the convergences of the last line of (5.48) hold. Assertion (5.48) is proved.

If $\vartheta>0$, by (5.10) and (5.47), the sequence $\left(\varepsilon \boldsymbol{e}\left(\boldsymbol{u}_{\varepsilon}\right) \mathbb{1}_{B_{\varepsilon}}\right)$ strongly converges to 0 in $L^{2}\left(\Omega \times(0, T) ; \mathbb{R}^{3}\right)$. On the other hand, by (4.4), (5.48) and Lemma 4.1, $\left(\varepsilon \boldsymbol{e}\left(\boldsymbol{u}_{\varepsilon}\right) \mathbb{1}_{B_{\varepsilon}}\right)$ two-scale converges to $\boldsymbol{e}_{y}\left(\boldsymbol{u}_{0}\right) \mathbb{1}_{B}$, hence $\boldsymbol{e}_{y}\left(\boldsymbol{u}_{0}\right)=0$ in $\Omega \times(0, T) \times B$ and, for a. e. $(x, t) \in \Omega \times(0, T)$, the restriction of $\boldsymbol{u}_{0}(x, t,$.$) to B$ is a rigid displacement. Since $\boldsymbol{u}_{0}$ is $Y$-periodic, we deduce that

$$
\boldsymbol{u}_{0}=\boldsymbol{a} \text { in } \Omega \times(0, T) \times B,
$$

for some $\boldsymbol{a} \in L^{\infty}\left(0, T ; L^{2}\left(\Omega ; \mathbb{R}^{3}\right)\right)$. By (4.4), (5.48) and Lemma 4.1 (i), the sequence $\left(\boldsymbol{u}_{\varepsilon} \mathbb{1}_{B_{\varepsilon}}\right)$ two-scale converges to $\boldsymbol{u}_{0}(x, t, y) \mathbb{1}_{B}(y)$. Fixing $\boldsymbol{\varphi} \in \mathcal{D}\left(\Omega \times(0, T) ; \mathbb{R}^{3}\right)$, taking $(5.11),(5.47)$ and (5.52) into account, and noticing that $\frac{\varepsilon}{r_{\varepsilon}} \rightarrow \frac{1}{|B|}$, we deduce

$$
\begin{aligned}
\int_{\Omega \times(0, T)} \boldsymbol{v} \cdot \boldsymbol{\varphi} d x d t & =\lim _{\varepsilon \rightarrow 0} \int_{\Omega \times(0, T)} \boldsymbol{u}_{\varepsilon} \cdot \boldsymbol{\varphi} d m_{\varepsilon} d t=\lim _{\varepsilon \rightarrow 0} \frac{\varepsilon}{r_{\varepsilon}} \int_{\Omega \times(0, T)} \boldsymbol{u}_{\varepsilon} \cdot \boldsymbol{\varphi} \mathbb{1}_{B_{\varepsilon}}(x) d x d t \\
& =\frac{1}{|B|} \int_{\Omega \times(0, T) \times B} \boldsymbol{u}_{0} \cdot \boldsymbol{\varphi}(x, t) 1_{B}(y) d x d t d y=\int_{\Omega \times(0, T)} \boldsymbol{a} \cdot \boldsymbol{\varphi} d x d t
\end{aligned}
$$

and infer, from the arbitrary choice of $\boldsymbol{\varphi}$, that $\boldsymbol{v}=\boldsymbol{a}$. Assertion (5.49) is proved.

Let us assume now that $\vartheta=0$ (i.e. that $r_{\varepsilon} \ll \varepsilon$ ). Since the stiff layers are periodicaly distributed, by (3.35) the field $\overline{\boldsymbol{v}}_{\varepsilon}$ defined by (5.20) takes the form

$$
\overline{\boldsymbol{v}}_{\varepsilon}(x, t):=\sum_{i \in Z_{\varepsilon}} \boldsymbol{u}_{\varepsilon}\left(x_{1}, x_{2}, \varepsilon i, t\right) \mathbb{1}_{\left(\varepsilon i-\frac{\varepsilon}{2}, \varepsilon i+\frac{\varepsilon}{2}\right]}\left(x_{3}\right),
$$

and coincides in $B_{\varepsilon}$ with the field $\hat{\boldsymbol{v}}_{\varepsilon}$ given by (5.17). Therefore, by (5.18),

$$
\int\left|\overline{\boldsymbol{v}}_{\varepsilon}-\boldsymbol{u}_{\varepsilon}\right|^{2}(\tau) d m_{\varepsilon} \leq C \frac{r_{\varepsilon}}{\varepsilon} \int_{\Omega} \varepsilon^{2}\left|\boldsymbol{e}\left(\boldsymbol{u}_{\varepsilon}\right)\right|^{2}(\tau) d x \quad \forall \tau \in[0, T]
$$

Since $r_{\varepsilon} \ll \varepsilon$, we deduce from (5.11), (5.47) and (5.54) that $\int\left|\overline{\boldsymbol{v}}_{\varepsilon}\right|^{2}(\tau) d m_{\varepsilon} \leq C$. On the other hand, taking (3.39), (3.35) and (5.53) into account, it is easy to check that $\int\left|\overline{\boldsymbol{v}}_{\varepsilon}\right|^{2}(\tau) d m_{\varepsilon}=\int_{\Omega}\left|\overline{\boldsymbol{v}}_{\varepsilon}\right|^{2}(\tau) d x$, therefore the sequence $\left(\overline{\boldsymbol{v}}_{\varepsilon}\right)$ is bounded in $L^{\infty}\left(0, T ; L^{2}\left(\Omega ; \mathbb{R}^{3}\right)\right)$. It then follows from Lemma 4.1 (ii) that

$$
\overline{\boldsymbol{v}}_{\varepsilon} \longrightarrow \overline{\boldsymbol{v}}_{0},
$$

up to a subsequence, for some $\overline{\boldsymbol{v}}_{0} \in L^{\infty}\left(0, T ; L^{2}\left(\Omega \times Y ; \mathbb{R}^{3}\right)\right)$. We establish below that

$$
\frac{\partial \overline{\boldsymbol{v}}_{0}}{\partial y_{3}}=0 \text { a.e. in } \Omega \times(0, T) \times Y, \quad \boldsymbol{u}_{0}=\overline{\boldsymbol{v}}_{0} \text { on } \Omega \times(0, T) \times \Sigma,
$$

and that (see (2.1)) 


$$
\begin{aligned}
& \boldsymbol{v}^{\prime}(x, t)=\overline{\boldsymbol{v}}_{0}^{\prime}(x, t, y) \quad \text { a.e. in } \Omega \times(0, T) \times Y, \\
& v_{3}(x, t)=\int_{\left(-\frac{1}{2}, \frac{1}{2}\right)^{2}} \bar{v}_{03}\left(x, t, s_{1}, s_{2}, y_{3}\right) d s_{1} d s_{2} .
\end{aligned}
$$

The next equation (proved below)

$$
\frac{\partial \bar{v}_{03}}{\partial y_{\alpha}}=0 \quad \forall \alpha \in\{1,2\}, \quad \text { if }(5.30) \text { holds true, }
$$

joined with (5.57), yields (5.50). It remains to prove (5.56), (5.57) and (5.58).

Proof of (5.56). Let us fix $\boldsymbol{\psi} \in \mathcal{D}\left(\Omega \times(0, T) ; \mathcal{D}_{\sharp}\left(Y ; \mathbb{R}^{3}\right)\right)$. By (5.53) we have

$$
\begin{aligned}
& \int_{\Omega \times(0, T)} \overline{\boldsymbol{v}}_{\varepsilon} \cdot \frac{\partial \boldsymbol{\psi}}{\partial y_{3}}\left(x, t, \frac{x}{\varepsilon}\right) d x d t \\
& =\sum_{i \in Z_{\varepsilon}} \int_{\Omega^{\prime} \times(0, T)} \boldsymbol{u}_{\varepsilon}\left(x_{1}, x_{2}, \varepsilon i, t\right) \cdot\left(\int_{\left(\varepsilon i-\frac{\varepsilon}{2}, \varepsilon i+\frac{\varepsilon}{2}\right)} \frac{\partial \boldsymbol{\psi}}{\partial y_{3}}\left(x, t, \frac{x^{\prime}}{\varepsilon}, \frac{x_{3}}{\varepsilon}\right) d x_{3}\right) d x^{\prime} d t .
\end{aligned}
$$

Since $\boldsymbol{\psi}(x, t,.) \in \mathcal{D}_{\sharp}\left(Y ; \mathbb{R}^{3}\right)$, the following holds

$$
\int_{\left(\varepsilon i-\frac{\varepsilon}{2}, \varepsilon i+\frac{\varepsilon}{2}\right)} \frac{\partial \boldsymbol{\psi}}{\partial y_{3}}\left(x, t, \frac{x^{\prime}}{\varepsilon}, \frac{x_{3}}{\varepsilon}\right) d x_{3}=\frac{1}{\varepsilon} \int_{\left(-\frac{1}{2}, \frac{1}{2}\right)} \frac{\partial \boldsymbol{\psi}}{\partial y_{3}}\left(x, t, \frac{x^{\prime}}{\varepsilon}, y_{3}\right) d y_{3}=0
$$

therefore $\int_{\Omega \times(0, T)} \overline{\boldsymbol{v}}_{\varepsilon} \cdot \frac{\partial \boldsymbol{\psi}}{\partial y_{3}}\left(x, t, \frac{x}{\varepsilon}\right) d x d t=0$. By passing to the limit as $\varepsilon \rightarrow 0$, we infer $\int_{\Omega \times(0, T) \times Y} \overline{\boldsymbol{v}}_{0} \cdot \frac{\partial \boldsymbol{\psi}}{\partial y_{3}}(x, t, y) d x d t d y=0$, and deduce from the arbitrary choice of $\boldsymbol{\psi}$ that

$$
\frac{\partial \bar{v}_{0}}{\partial y_{3}}=0 \quad \text { in } \quad \Omega \times(0, T) \times Y .
$$

We set $Y^{+}:=\left(-\frac{1}{2}, \frac{1}{2}\right)^{2} \times\left(0, \frac{1}{2}\right)$ and fix $\boldsymbol{\Psi} \in \mathcal{D}\left(\Omega \times(0, T) ; \mathcal{D}_{\sharp}\left(Y ; \mathbb{S}^{3}\right)\right)$. Then, for each $i \in Z_{\varepsilon}$ (defined by $\left.(3.15)\right)$, the field $\boldsymbol{\Psi}\left(x, t, \frac{x}{\varepsilon}\right)$ vanishes on $\partial\left(\Omega^{\prime} \times\left(\varepsilon i-\frac{\varepsilon}{2}, \varepsilon i+\frac{\varepsilon}{2}\right)\right) \times$ $(0, T)$, and, for $\varepsilon$ small enough, the support of $\boldsymbol{\Psi}\left(x, t, \frac{x}{\varepsilon}\right)$ is included in $\bigcup_{i \in Z_{\varepsilon}} \Omega^{\prime} \times$ $\left[\varepsilon i-\frac{\varepsilon}{2}, \varepsilon i+\frac{\varepsilon}{2}\right] \times(0, T)$. Hence, by integration by parts, we get

$$
\begin{aligned}
& \int_{\Omega \times(0, T)} \varepsilon \boldsymbol{e}\left(\boldsymbol{u}_{\varepsilon}\right): \boldsymbol{\Psi}\left(x, t, \frac{x}{\varepsilon}\right) \mathbb{1}_{Y_{\sharp}^{+}}\left(\frac{x}{\varepsilon}\right) d x d t=\sum_{i \in Z_{\varepsilon}} \int_{\Omega^{\prime} \times\left(\varepsilon i, \varepsilon i+\frac{\varepsilon}{2}\right) \times(0, T)} \underset{e\left(\boldsymbol{u}_{\varepsilon}\right): \boldsymbol{\Psi}}{ }\left(x, t, \frac{x}{\varepsilon}\right) d x d t \\
& =-\sum_{i \in Z_{\varepsilon}} \varepsilon \int_{\Omega^{\prime} \times\{\varepsilon i\} \times(0, T)} \boldsymbol{u}_{\varepsilon} \cdot \boldsymbol{\Psi}\left(x, t, \frac{x^{\prime}}{\varepsilon}, 0\right) \boldsymbol{e}_{3} d \mathcal{H}^{2}(x) d t \\
& -\int_{\Omega \times(0, T)} \varepsilon \boldsymbol{u}_{\varepsilon} \cdot \operatorname{div}_{x} \boldsymbol{\Psi}\left(x, t, \frac{x}{\varepsilon}\right) \mathbb{1}_{Y_{\sharp}^{+}}\left(\frac{x}{\varepsilon}\right)+\boldsymbol{u}_{\varepsilon} \cdot \operatorname{div}_{y} \boldsymbol{\Psi}\left(x, t, \frac{x}{\varepsilon}\right) \mathbb{1}_{Y_{\sharp}^{+}}\left(\frac{x}{\varepsilon}\right) d x d t .
\end{aligned}
$$

We set $\overline{\boldsymbol{\Psi}}_{\varepsilon}\left(x, t, \frac{x^{\prime}}{\varepsilon}, 0\right):=\sum_{i \in Z_{\varepsilon}} \boldsymbol{\Psi}\left(x_{1}, x_{2}, \varepsilon i, t, \frac{x^{\prime}}{\varepsilon}, 0\right) \mathbb{1}_{\left(\varepsilon i-\frac{\varepsilon}{2}, \varepsilon i+\frac{\varepsilon}{2}\right)}\left(x_{3}\right)$. Notice that 


$$
\begin{aligned}
& \left|\overline{\mathbf{\Psi}}_{\varepsilon}\left(x, t, \frac{x^{\prime}}{\varepsilon}, 0\right)-\mathbf{\Psi}\left(x, t, \frac{x^{\prime}}{\varepsilon}, 0\right)\right| \leq C \varepsilon, \\
& \left|\frac{\partial}{\partial y_{\alpha}} \overline{\mathbf{\Psi}}_{\varepsilon}\left(x, t, \frac{x^{\prime}}{\varepsilon}, 0\right)-\frac{\partial}{\partial y_{\alpha}} \mathbf{\Psi}\left(x, t, \frac{x^{\prime}}{\varepsilon}, 0\right)\right| \leq C \varepsilon \quad \text { for } \quad \alpha \in\{1,2\} .
\end{aligned}
$$

By the definitions $\overline{\boldsymbol{\Psi}}_{\varepsilon}$ and $\overline{\boldsymbol{v}}_{\varepsilon}$ (see (5.53)), there holds

$$
-\sum_{i \in Z_{\varepsilon}} \varepsilon \int_{\Omega^{\prime} \times\{\varepsilon i\} \times(0, T)} \boldsymbol{u}_{\varepsilon} \cdot \mathbf{\Psi}\left(x, t, \frac{x^{\prime}}{\varepsilon}, 0\right) \boldsymbol{e}_{3} d \mathcal{H}^{2}(x) d t=-\int_{\Omega \times(0, T)} \overline{\boldsymbol{v}}_{\varepsilon} \cdot \overline{\mathbf{\Psi}}_{\varepsilon}\left(x, t, \frac{x^{\prime}}{\varepsilon}, 0\right) \boldsymbol{e}_{3} d x d t .
$$

Taking (5.55) and (5.61) into account, and noticing that by (5.59) we have $\overline{\boldsymbol{v}}_{0}(x, t, y)=$ $\overline{\boldsymbol{v}}_{0}\left(x, t, y^{\prime}, 0\right)$ in $\Omega \times(0, T) \times Y$, we obtain

$$
\begin{aligned}
\lim _{\varepsilon \rightarrow 0}-\int_{\Omega \times(0, T)} \overline{\boldsymbol{v}}_{\varepsilon} \cdot \overline{\mathbf{\Psi}}_{\varepsilon}\left(x, t, \frac{x^{\prime}}{\varepsilon}, 0\right) \boldsymbol{e}_{3} d x d t & =-\int_{\Omega \times(0, T) \times Y} \overline{\boldsymbol{v}}_{0} \cdot \mathbf{\Psi}\left(x, t, y^{\prime}, 0\right) \boldsymbol{e}_{3} d x d t d y \\
& =-\int_{\Omega \times(0, T) \times \Sigma} \overline{\boldsymbol{v}}_{0} \cdot \mathbf{\Psi} \boldsymbol{e}_{3} d x d t d \mathcal{H}^{2}(y) .
\end{aligned}
$$

By passing to the limit as $\varepsilon \rightarrow 0$ in (5.60), applying Lemma 4.1 (i) with $h_{\varepsilon}=\mathbb{1}_{Y^{+}}\left(\frac{x}{\varepsilon}\right)$ and taking (5.48), (5.62) and (5.63) into account, we get

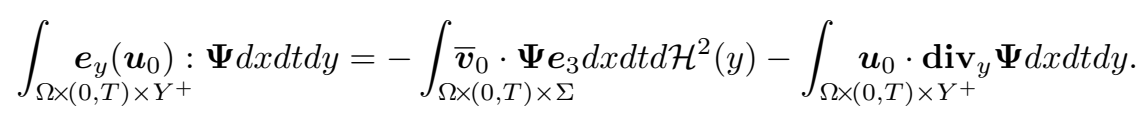

By integration by parts, we have

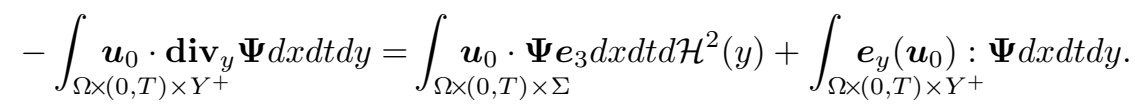

Joining the last two equations, we infer that $\int_{\Omega \times(0, T) \times \Sigma} \boldsymbol{u}_{0} \cdot \boldsymbol{\Psi} \boldsymbol{e}_{3} d x d t d \mathcal{H}^{2}(y)=$ $\int_{\Omega \times(0, T) \times \Sigma} \overline{\boldsymbol{v}}_{0} \cdot \boldsymbol{\Psi} \boldsymbol{e}_{3} d x d t d \mathcal{H}^{2}(y)$. By the arbitrary choice of $\boldsymbol{\Psi}$ (and by (5.59)), we deduce that (5.56) holds.

Proof of (5.57). Let us fix $\boldsymbol{\psi} \in \mathcal{D}\left(\Omega \times(0, T) ; \mathbb{R}^{3}\right)$ and set

$$
\overline{\boldsymbol{\psi}}_{\varepsilon}(x, t):=\sum_{i \in Z_{\varepsilon}} \boldsymbol{\psi}\left(x_{1}, x_{2}, \varepsilon i, t\right) \mathbb{1}_{\left(\varepsilon i-\frac{\varepsilon}{2}, \varepsilon i+\frac{\varepsilon}{2}\right]}\left(x_{3}\right) .
$$

By (3.39) and (5.53) we have

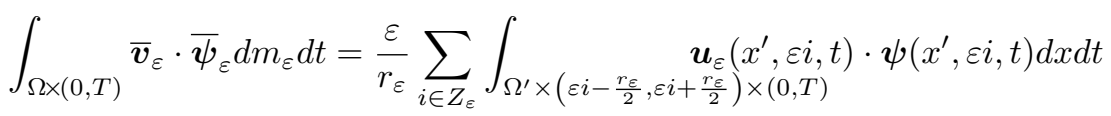

$$
\begin{aligned}
& =\sum_{i \in Z_{\varepsilon}} \int_{\Omega^{\prime} \times\left(\varepsilon i-\frac{\varepsilon}{2}, \varepsilon i+\frac{\varepsilon}{2}\right) \times(0, T)} \boldsymbol{u}_{\varepsilon}\left(x^{\prime}, \varepsilon i, t\right) \cdot \boldsymbol{\psi}\left(x^{\prime}, \varepsilon i, t\right) d x d t=\int_{\Omega \times(0, T)} \overline{\boldsymbol{v}}_{\varepsilon} \cdot \overline{\boldsymbol{\psi}}_{\varepsilon} d x d t .
\end{aligned}
$$

We infer from (5.55) and from the estimate 


$$
\left|\psi-\bar{\psi}_{\varepsilon}\right|_{L^{\infty}\left(\Omega \times(0, T) ; \mathbb{R}^{3}\right)} \leq C \varepsilon,
$$

that

$$
\lim _{\varepsilon \rightarrow 0} \int_{\Omega \times(0, T)} \overline{\boldsymbol{v}}_{\varepsilon} \cdot \overline{\boldsymbol{\psi}}_{\varepsilon} d x d t=\int_{\Omega \times(0, T)}\left(\int_{Y} \overline{\boldsymbol{v}}_{0}(x, t, y) d y\right) \boldsymbol{\psi}(x, t) d x d t .
$$

By (5.54) and (5.66), the following holds

$$
\lim _{\varepsilon \rightarrow 0}\left|\int_{\Omega \times(0, T)} \boldsymbol{u}_{\varepsilon} \cdot \boldsymbol{\psi} d m_{\varepsilon} d t-\int_{\Omega \times(0, T)} \overline{\boldsymbol{v}}_{\varepsilon} \cdot \overline{\boldsymbol{\psi}}_{\varepsilon} d m_{\varepsilon} d t\right|=0
$$

The weak* convergence of $\left(\boldsymbol{u}_{\varepsilon} m_{\varepsilon}\right)$ to $\boldsymbol{v}$ and (5.65), (5.67), (5.68), imply

$$
\int_{\Omega \times(0, T)}^{\boldsymbol{v} \cdot \boldsymbol{\psi}} \boldsymbol{\psi} d x d t=\lim _{\varepsilon \rightarrow 0} \int_{\Omega \times(0, T)} \boldsymbol{u}_{\varepsilon} \cdot \boldsymbol{\psi} d m_{\varepsilon} d t=\int_{\Omega \times(0, T)}\left(\int_{Y} \overline{\boldsymbol{v}}_{0}(x, t, y) d y\right) \cdot \boldsymbol{\psi}(x, t) d x d t
$$

yielding, by the arbitrary choice of $\boldsymbol{\psi}$,

$$
\boldsymbol{v}(x, t)=\int_{Y} \overline{\boldsymbol{v}}_{0}(x, t, y) d y \quad \text { in } \quad \Omega \times(0, T) .
$$

By (5.59) and (5.69), the proof of (5.57) is achieved provided that we establish that

$$
\frac{\partial \bar{v}_{0 \alpha}}{\partial y_{\beta}}=0 \quad \forall \alpha, \beta \in\{1,2\}
$$

To that aim, let us fix $\boldsymbol{\Psi} \in \mathcal{D}\left(\Omega \times(0, T) ; C_{\sharp}^{\infty}\left(\left(-\frac{1}{2}, \frac{1}{2}\right)^{2} ; \mathbb{S}^{3}\right)\right)$. Since $\boldsymbol{u}_{\varepsilon}$ vanishes on $\partial \Omega \times(0, T)$, by integrating by parts with respect to $x_{1}$ and $x_{2}$, we get (see (2.1))

$$
\begin{aligned}
\int_{\Omega \times(0, T)} \boldsymbol{e}_{x^{\prime}}\left(\boldsymbol{u}_{\varepsilon}^{\prime}\right): \Psi\left(x, t, \frac{x^{\prime}}{\varepsilon}\right) d m_{\varepsilon} d t= & -\int_{\Omega \times(0, T)} \boldsymbol{u}_{\varepsilon}^{\prime} \cdot \operatorname{div}_{x^{\prime}}^{\prime} \mathbf{\Psi}\left(x, t, \frac{x^{\prime}}{\varepsilon}\right) d m_{\varepsilon} d t \\
& -\frac{1}{\varepsilon} \int_{\Omega \times(0, T)} \boldsymbol{u}_{\varepsilon}^{\prime} \cdot \operatorname{div}_{y^{\prime}}^{\prime} \mathbf{\Psi}\left(x, t, \frac{x^{\prime}}{\varepsilon}\right) d m_{\varepsilon} d t .
\end{aligned}
$$

By (5.10), the left-hand side and the first term on the right-hand side of the above equation are bounded, therefore

$$
\lim _{\varepsilon \rightarrow 0} \int_{\Omega \times(0, T)} \boldsymbol{u}_{\varepsilon}^{\prime} \cdot \operatorname{div}_{y^{\prime}}^{\prime} \mathbf{\Psi}\left(x, t, \frac{x^{\prime}}{\varepsilon}\right) d m_{\varepsilon} d t=0 .
$$

On the other hand, by (5.54) and (5.61), there holds

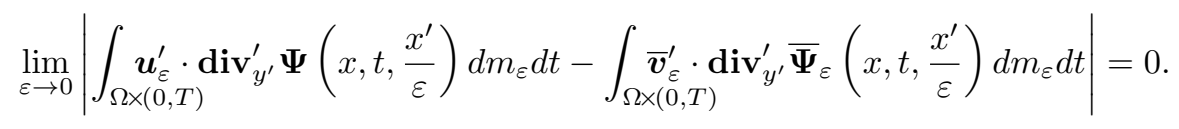


A computation analogous to (5.65) yields

$$
\int_{\Omega \times(0, T)} \overline{\boldsymbol{v}}_{\varepsilon}^{\prime} \cdot \operatorname{div}_{y^{\prime}}^{\prime} \overline{\mathbf{\Psi}}_{\varepsilon}\left(x, t, \frac{x^{\prime}}{\varepsilon}\right) d m_{\varepsilon} d t=\int_{\Omega \times(0, T)} \overline{\boldsymbol{v}}_{\varepsilon}^{\prime} \cdot \operatorname{div}_{y^{\prime}}^{\prime} \overline{\mathbf{\Psi}}_{\varepsilon}\left(x, t, \frac{x^{\prime}}{\varepsilon}\right) d x d t .
$$

By (5.61) and by the two-scale convergence of $\overline{\boldsymbol{v}}_{\varepsilon}$ to $\overline{\boldsymbol{v}}_{0}$ (see (5.55)), there holds

$$
\lim _{\varepsilon \rightarrow 0} \int_{\Omega \times(0, T)} \overline{\boldsymbol{v}}_{\varepsilon}^{\prime} \cdot \operatorname{div}_{y^{\prime}}^{\prime} \bar{\Psi}_{\varepsilon}\left(x, t, \frac{x^{\prime}}{\varepsilon}\right) d x d t=\int_{\Omega \times(0, T) \times Y} \overline{\boldsymbol{v}}_{0}^{\prime} \cdot \operatorname{div}_{y^{\prime}}^{\prime} \mathbf{\Psi}\left(x, t, y^{\prime}\right) d x d t d y .
$$

Combining (5.71)-(5.74), we get

$$
\int_{\Omega \times(0, T) \times Y} \overline{\boldsymbol{v}}_{0}^{\prime} \cdot \operatorname{div}_{y^{\prime}}^{\prime} \mathbf{\Psi}\left(x, t, y^{\prime}\right) d x d t d y=0,
$$

hence $\boldsymbol{e}_{y^{\prime}}\left(\overline{\boldsymbol{v}}_{0}^{\prime}\right)=0$, in the sense of distributions. We deduce that $y^{\prime} \rightarrow \overline{\boldsymbol{v}}_{0}^{\prime}\left(x, t, y^{\prime}, y_{3}\right)$ is a rigid displacement. By integrating (5.75) by parts, we infer

$$
\int_{\Omega \times(0, T) \times \partial\left(-\frac{1}{2}, \frac{1}{2}\right)^{2}} \overline{\boldsymbol{v}}_{0}^{\prime}(x, t, y) \cdot \boldsymbol{\Psi}\left(x, t, y^{\prime}\right) \boldsymbol{\nu} d x d t d \mathcal{H}^{1}\left(y^{\prime}\right)=0,
$$

and deduce from the arbitrary choice of $\boldsymbol{\Psi} \in \mathcal{D}\left(\Omega \times(0, T) ; C_{\sharp}^{\infty}\left(\left(-\frac{1}{2}, \frac{1}{2}\right)^{2} ; \mathbb{S}^{3}\right)\right)$, that $\overline{\boldsymbol{v}}_{0}^{\prime} \in L^{2}\left(\Omega \times(0, T), H_{\sharp}^{1}\left(\left(-\frac{1}{2}, \frac{1}{2}\right)^{2} ; \mathbb{R}^{3}\right)\right)$. The periodicity of $\overline{\boldsymbol{v}}_{0}^{\prime}$ with respect to $y^{\prime}$ and the fact that $y^{\prime} \rightarrow \overline{\boldsymbol{v}}_{0}^{\prime}\left(x, t, y^{\prime}, y_{3}\right)$ is a rigid displacement imply that $y^{\prime} \rightarrow \overline{\boldsymbol{v}}_{0}^{\prime}\left(x, t, y^{\prime}\right)$ is a constant field. Assertion (5.70) is proved. The proof of (5.57) is achieved.

Proof of (5.58). We assume (5.30), fix $\psi \in \mathcal{D}\left(\Omega \times(0, T) ; \mathcal{D}_{\sharp}\left(\left(-\frac{1}{2}, \frac{1}{2}\right)^{2}\right)\right), \eta \in \mathcal{D}(I)$, and $\alpha \in\{1,2\}$. Noticing that the mapping $x \rightarrow \psi\left(x, t, \frac{x^{\prime}}{\varepsilon}\right) \eta\left(\frac{y_{\varepsilon}\left(x_{3}\right)}{r_{\varepsilon}}\right)$ is compactly supported in $B_{\varepsilon}$, by integration by parts we obtain

$$
\begin{aligned}
\int_{\Omega \times(0, T)}\left(\frac{\partial u_{\varepsilon \alpha}}{\partial x_{3}}+\frac{\partial u_{\varepsilon 3}}{\partial x_{\alpha}}\right) \psi\left(x, t, \frac{x^{\prime}}{\varepsilon}\right) \eta\left(\frac{y_{\varepsilon}\left(x_{3}\right)}{r_{\varepsilon}}\right) d m_{\varepsilon} d t \\
=-\int_{\Omega \times(0, T)}\left(u_{\varepsilon \alpha} \frac{\partial \psi}{\partial x_{3}}\left(x, t, \frac{x^{\prime}}{\varepsilon}\right)+u_{\varepsilon 3} \frac{\partial \psi}{\partial x_{\alpha}}\left(x, t, \frac{x^{\prime}}{\varepsilon}\right)\right) \eta\left(\frac{y_{\varepsilon}\left(x_{3}\right)}{r_{\varepsilon}}\right) d m_{\varepsilon} d t \\
\quad-\int_{\Omega \times(0, T)} \frac{u_{\varepsilon \alpha}}{r_{\varepsilon}} \psi\left(x, t, \frac{x^{\prime}}{\varepsilon}\right) \frac{\partial \eta}{\partial x_{3}}\left(\frac{y_{\varepsilon}\left(x_{3}\right)}{r_{\varepsilon}}\right) d m_{\varepsilon} d t \\
-\frac{1}{\varepsilon} \int_{\Omega \times(0, T)} u_{\varepsilon 3} \frac{\partial \psi}{\partial y_{\alpha}}\left(x, t, \frac{x^{\prime}}{\varepsilon}\right) \eta\left(\frac{y_{\varepsilon}\left(x_{3}\right)}{r_{\varepsilon}}\right) d m_{\varepsilon} d t .
\end{aligned}
$$

By (5.1) we have

$$
\int\left|\frac{\partial u_{\varepsilon \alpha}}{\partial x_{3}}+\frac{\partial u_{\varepsilon 3}}{\partial x_{\alpha}}\right|^{2}+\left|\frac{u_{\varepsilon \alpha}}{r_{\varepsilon}}\right|^{2}+\left|u_{\varepsilon 3}\right|^{2}(\tau) d m_{\varepsilon} \leq C \int \frac{1}{r_{\varepsilon}^{2}}\left|\boldsymbol{e}\left(\boldsymbol{u}_{\varepsilon}\right)\right|^{2}(\tau) d m_{\varepsilon},
$$

hence, by (5.30), all terms of the three first lines of (5.76) are bounded. We infer

$$
\lim _{\varepsilon \rightarrow 0} \int_{\Omega \times(0, T)} u_{\varepsilon 3} \frac{\partial \psi}{\partial y_{\alpha}}\left(x, t, \frac{x^{\prime}}{\varepsilon}\right) \eta\left(\frac{y_{\varepsilon}\left(x_{3}\right)}{r_{\varepsilon}}\right) d m_{\varepsilon} d t=0,
$$


and then deduce from (5.54) and from an estimate analogous to (5.61) that

$$
\lim _{\varepsilon \rightarrow 0} \int_{\Omega \times(0, T)} \bar{v}_{\varepsilon 3} \frac{\partial \bar{\psi}_{\varepsilon}}{\partial y_{\alpha}}\left(x, t, \frac{x^{\prime}}{\varepsilon}\right) \eta\left(\frac{y_{\varepsilon}\left(x_{3}\right)}{r_{\varepsilon}}\right) d m_{\varepsilon} d t=0,
$$

where $\bar{\psi}_{\varepsilon}$ is defined by (5.64). Taking (5.53) and (5.64) into account, and noticing that $\frac{1}{r_{\varepsilon}} \int_{\left(\varepsilon i-\frac{r_{\varepsilon}}{2}, \varepsilon i+\frac{r_{\varepsilon}}{2}\right)} \eta\left(\frac{y_{\varepsilon}\left(x_{3}\right)}{r_{\varepsilon}}\right) d x_{3}=\int_{I} \eta(y) d y$, we get

$$
\begin{aligned}
& \int_{\Omega \times(0, T)} \bar{v}_{\varepsilon 3} \frac{\partial \bar{\psi}_{\varepsilon}}{\partial y_{\alpha}}\left(x, t, \frac{x^{\prime}}{\varepsilon}\right) \eta\left(\frac{y_{\varepsilon}\left(x_{3}\right)}{r_{\varepsilon}}\right) d m_{\varepsilon} d t \\
& =\frac{\varepsilon}{r_{\varepsilon}} \sum_{i \in Z_{\varepsilon}} \int_{\Omega^{\prime} \times\left(\varepsilon i-\frac{r_{\varepsilon}}{2}, \varepsilon i+\frac{r_{\varepsilon}}{2}\right) \times(0, T)} u_{\varepsilon 3}\left(x^{\prime}, \varepsilon i, t\right) \frac{\partial \psi}{\partial y_{\alpha}}\left(x^{\prime}, \varepsilon i, t, \frac{x^{\prime}}{\varepsilon}\right) \eta\left(\frac{y_{\varepsilon}\left(x_{3}\right)}{r_{\varepsilon}}\right) d x^{\prime} d x_{3} d t \\
& =\varepsilon \sum_{i \in Z_{\varepsilon}} \int_{\Omega^{\prime} \times(0, T)} u_{\varepsilon 3}\left(x^{\prime}, \varepsilon i, t\right) \frac{\partial \psi}{\partial y_{\alpha}}\left(x^{\prime}, \varepsilon i, t, \frac{x^{\prime}}{\varepsilon}\right) d x^{\prime} d t\left(\int_{I} \eta(y) d y\right) \\
& =\left(\int_{\Omega \times(0, T)} \bar{v}_{\varepsilon 3} \frac{\partial \bar{\psi}_{\varepsilon}}{\partial y_{\alpha}}\left(x, t, \frac{x^{\prime}}{\varepsilon}\right) d x d t\right)\left(\int_{I} \eta(y) d y\right) .
\end{aligned}
$$

On the other hand, by (5.55) and an estimate analogous to (5.61), there holds

$$
\lim _{\varepsilon \rightarrow 0} \int_{\Omega \times(0, T)} \bar{v}_{\varepsilon 3} \frac{\partial \bar{\psi}_{\varepsilon}}{\partial y_{\alpha}}\left(x, t, \frac{x^{\prime}}{\varepsilon}\right) d x d t=\int_{\Omega \times(0, T) \times Y} \boldsymbol{v}_{03} \frac{\partial \psi}{\partial y_{\alpha}} d x d t d y .
$$

Joining (5.77), (5.78), (5.79), and choosing $\eta$ such that $\int_{I} \eta d y_{3} \neq 0$, we infer that $\int_{\Omega \times(0, T) \times Y} v_{03} \frac{\partial \psi}{\partial y_{\alpha}} d x d t d y=0$. By the arbitraryness of $\psi$, Assertion $(5.58)$ is proved. In the next Corollary, we derive from Proposition 5.2 and lemmas 5.3, 5.4, 5.5, a series of convergences and identification relations for various sequences associated with the solution to (3.5).

Corollary 5.6. Let $\boldsymbol{u}_{\varepsilon}$ be the solution to (3.5).

(i) Up to a subsequence, the convergences (5.11) hold and

$$
n v_{1}=n v_{2}=0 \quad \text { if } \quad k=+\infty, \quad n \boldsymbol{v}=0 \quad \text { if } \quad \kappa=+\infty .
$$

Under (5.13) or (5.14), $n \boldsymbol{u}=n \boldsymbol{v}$.

(ii) If $\kappa>0$, the relation (5.31) and convergences (5.32) hold.

(iii) In the periodic case, that is under (3.34) and (3.35), the convergences and relations (5.48) hold. If $\vartheta>0$ (resp. $\vartheta=0)$, the relations (5.49) (resp. (5.50)) are verified.

Proof. Noticing that by (3.8) and (5.5), the estimate (5.10) holds, Assertion (i) follows from Lemma 5.3 (Assertion (5.80) is a consequence of (3.8), (5.5), and (5.12)). If $\kappa>0$, by (3.8) and (5.5) the estimate (5.30) holds, and Assertion (ii) follows from Lemma 5.4. In the periodic case, by (3.34), (3.35), and (5.5), $\boldsymbol{u}_{\varepsilon}$ satisfies (5.10) and (5.47) and by (3.8), (3.38) and (5.5) it satisfies (5.30) if $\vartheta=0$. Hence (iii) results from Lemma 5.5. 
6. Proof of theorems 3.1, 3.7, 3.12.

In the spirit of Tartar [53,54], we will multiply (3.5) by an appropriate test field $\phi_{\varepsilon}$, integrate by parts, and, passing to the limit as $\varepsilon \rightarrow 0$ by means of the convergences derived in Corollary 5.6, obtain a variational problem equivalent to the announced limit problem, and also to (4.23) for some suitable $H, V, a, h, \xi_{0}, \xi_{1}$. Theorem 4.4 will yield existence, uniqueness, and regularity of the effective displacement. Uniqueness implies that the convergences obtained in Corollary 5.6 for subsequences hold for the complete sequences.

6.1. Proof of Theorem 3.12. We set

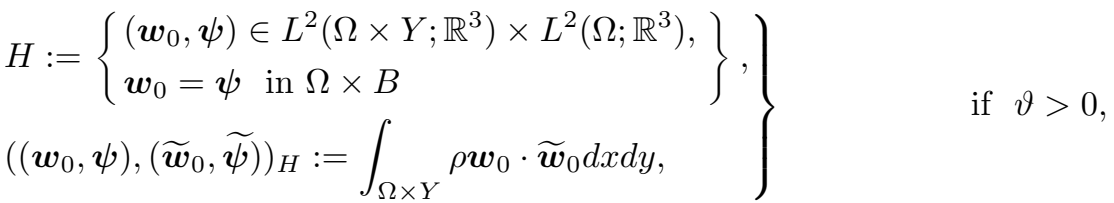

$$
\begin{aligned}
& \left.\begin{array}{l}
H:=L^{2}\left(\Omega \times Y ; \mathbb{R}^{3}\right) \times L^{2}\left(\Omega ; \mathbb{R}^{3}\right), \\
\left(\left(\boldsymbol{w}_{0}, \boldsymbol{\psi}\right),\left(\widetilde{\boldsymbol{w}}_{0}, \widetilde{\boldsymbol{\psi}}\right)\right)_{H}:=\int_{\Omega \times Y} \rho \boldsymbol{w}_{0} \cdot \widetilde{\boldsymbol{w}}_{0} d x d y+\int_{\Omega} \bar{\rho}_{1} \boldsymbol{\psi} \cdot \widetilde{\boldsymbol{\psi}} d x,
\end{array}\right\} \quad \text { if } \vartheta=0 .
\end{aligned}
$$

We easily deduce from the positiveness of $\rho$ and $\bar{\rho}_{1}$ (see (3.7)) that $H$ is a Hilbert space. We fix a couple $\left(\boldsymbol{w}_{0}, \boldsymbol{\psi}\right) \in L^{2}(0, T ; H)$ satisfying (see (3.37))

$$
\begin{aligned}
& \boldsymbol{w}_{0} \in C^{\infty}\left([0, T] ; \mathcal{D}\left(\Omega ; C_{\sharp}^{\infty}\left(Y ; \mathbb{R}^{3}\right)\right)\right), \quad \boldsymbol{\psi} \in C^{\infty}\left([0, T] ; \mathcal{D}\left(\Omega ; \mathbb{R}^{3}\right)\right), \\
& \boldsymbol{w}_{0}(T)=\frac{\partial \boldsymbol{w}_{0}}{\partial t}(T)=\boldsymbol{\psi}(T)=\frac{\partial \boldsymbol{\psi}}{\partial t}(T)=0, \\
& \psi_{1}=\psi_{2}=0 \quad \text { if } \quad k=+\infty, \quad \boldsymbol{\psi}=0 \quad \text { if } \quad \kappa=+\infty \\
& \boldsymbol{w}_{0}(., y)=\boldsymbol{\psi}(.) \quad \text { in } \Omega \times(0, T) \times A .
\end{aligned}
$$

We choose a sequence $\left(\alpha_{\varepsilon}\right)$ of positive reals such that

$$
\varepsilon \ll \alpha_{\varepsilon} \ll 1 \quad \text { and } \quad\left[\frac{r_{\varepsilon}}{\varepsilon} \ll \alpha_{\varepsilon} \ll 1 \quad \text { if } \vartheta=0\right]
$$

and set

$$
C_{\varepsilon}:=\left\{x \in \Omega, \operatorname{dist}\left(x, B_{\varepsilon}\right)<\alpha_{\varepsilon} r_{\varepsilon}\right\} .
$$

It is usefull to notice that

$$
\mathcal{L}^{3}\left(C_{\varepsilon} \backslash B_{\varepsilon}\right) \leq C \frac{\alpha_{\varepsilon} r_{\varepsilon}}{\varepsilon},
$$

and that, by (6.5), the following estimate holds for $m \in\{1,2\}$ :

$$
\begin{aligned}
& \left|\boldsymbol{\psi}(x, t)-\boldsymbol{w}_{0}\left(x, t, \frac{x}{\varepsilon}\right)\right|+\left|\frac{\partial^{m} \boldsymbol{\psi}}{\partial t^{m}}(x, t)-\frac{\partial^{m} \boldsymbol{w}_{0}}{\partial t^{m}}\left(x, t, \frac{x}{\varepsilon}\right)\right| \leq C \beta_{\varepsilon} \quad \text { in } C_{\varepsilon} \times(0, T), \\
& \beta_{\varepsilon}=\alpha_{\varepsilon} \quad \text { if } \vartheta>0, \quad \beta_{\varepsilon}=\frac{r_{\varepsilon}}{\varepsilon} \quad \text { if } \vartheta=0 .
\end{aligned}
$$


By (6.7), we can fix a sequence $\left(\eta_{\varepsilon}\right)$ in $C^{\infty}(\bar{\Omega})$ satisfying

$$
0 \leq \eta_{\varepsilon} \leq 1, \quad \eta_{\varepsilon}=1 \quad \text { in } \quad B_{\varepsilon}, \quad \eta_{\varepsilon}=0 \quad \text { in } \quad \Omega \backslash C_{\varepsilon}, \quad\left|\nabla \eta_{\varepsilon}\right|<\frac{C}{r_{\varepsilon} \alpha_{\varepsilon}}
$$

The sequence of test fields $\left(\phi_{\varepsilon}\right)$ mentioned above will be defined by

$$
\phi_{\varepsilon}(x, t):=\eta_{\varepsilon}(x) \widehat{\boldsymbol{\psi}}_{\varepsilon}(x, t)+\left(1-\eta_{\varepsilon}(x)\right) \boldsymbol{w}_{0}\left(x, t, \frac{x}{\varepsilon}\right),
$$

where $\widehat{\boldsymbol{\psi}}_{\varepsilon}$ is described in Section 7. As $\boldsymbol{\phi}_{\varepsilon}(x, t)=\boldsymbol{w}_{0}\left(x, t, \frac{x}{\varepsilon}\right)$ in $\Omega \backslash C_{\varepsilon} \times(0, T)$, we deduce from (6.5), (6.9), (6.10), (6.11), and (7.4) that the following estimates hold in $\Omega \times(0, T)$ for $m \in\{1,2\}$ :

$$
\begin{aligned}
& \left|\boldsymbol{\phi}_{\varepsilon}(x, t)-\boldsymbol{w}_{0}\left(x, t, \frac{x}{\varepsilon}\right)\right|+\left|\frac{\partial^{m} \boldsymbol{\phi}_{\varepsilon}}{\partial t^{m}}(x, t)-\frac{\partial^{m} \boldsymbol{w}_{0}}{\partial t^{m}}\left(x, t, \frac{x}{\varepsilon}\right)\right| \leq C \beta_{1 \varepsilon}, \\
& \beta_{1 \varepsilon}=r_{\varepsilon}+\alpha_{\varepsilon} \quad \text { if } \vartheta>0, \quad \beta_{1 \varepsilon}=\frac{r_{\varepsilon}}{\varepsilon} \quad \text { if } \quad \vartheta=0 .
\end{aligned}
$$

It is also interesting to notice that by (6.10), (6.11), and (7.4),

$$
\left|\boldsymbol{\phi}_{\varepsilon}(x, t)-\boldsymbol{\psi}(x, t)\right|+\left|\frac{\partial^{m} \boldsymbol{\phi}_{\varepsilon}}{\partial t^{m}}(x, t)-\frac{\partial^{m} \boldsymbol{\psi}}{\partial t^{m}}(x, t)\right| \leq C r_{\varepsilon} \quad \text { in } \quad B_{\varepsilon} \times(0, T) .
$$

By (3.5) and (3.6) we have $\left|\boldsymbol{\sigma}_{\varepsilon}\left(\phi_{\varepsilon}\right)\right| \leq C \mu_{0 \varepsilon}\left|\nabla \phi_{\varepsilon}\right|$ in $\left(\Omega \backslash B_{\varepsilon}\right) \times(0, T)$, therefore by (6.9), (6.10), (6.12), (7.4), the next estimates are satisfied in $\left(C_{\varepsilon} \backslash B_{\varepsilon}\right) \times(0, T)$

$$
\begin{aligned}
&\left|\boldsymbol{\sigma}_{\varepsilon}\left(\boldsymbol{\phi}_{\varepsilon}\right)\right| \leq C \mu_{0 \varepsilon}\left(\left|\nabla \eta_{\varepsilon}\right|\left|\widehat{\boldsymbol{\psi}}_{\varepsilon}(x, t)-\boldsymbol{w}_{0}\left(x, t, \frac{x}{\varepsilon}\right)\right|+\left|\nabla \widehat{\boldsymbol{\psi}}_{\varepsilon}(x, t)\right|+\left|\boldsymbol{\nabla}\left(\boldsymbol{w}_{0}\left(x, t, \frac{x}{\varepsilon}\right)\right)\right|\right) \\
& \leq C \mu_{0 \varepsilon}\left(\frac{1}{\alpha_{\varepsilon} r_{\varepsilon}}\left(r_{\varepsilon}+\beta_{\varepsilon}\right)+\frac{C}{\varepsilon}\right) \leq C \mu_{0 \varepsilon} \beta_{2 \varepsilon}, \\
& \beta_{2 \varepsilon}=\frac{1}{\alpha_{\varepsilon}}+\frac{1}{\varepsilon} \quad \text { if } \vartheta>0, \quad \beta_{2 \varepsilon}=\frac{1}{\alpha_{\varepsilon} \varepsilon} \quad \text { if } \vartheta=0 .
\end{aligned}
$$

Applying (4.5) to $\chi_{\varepsilon}=\rho_{\varepsilon} \mathbb{1}_{\Omega \backslash B_{\varepsilon}}, h_{0} \in\left\{\boldsymbol{w}_{0}, \frac{\partial^{2} \boldsymbol{w}_{0}}{\partial t^{2}}, \boldsymbol{w}_{0}(0), \frac{\partial^{2} \boldsymbol{w}_{0}}{\partial t^{2}}(0)\right\}$, we deduce from (3.7), (4.4), (6.6), and (6.12), that the following convergences hold for $m \in\{1,2\}$

$$
\begin{array}{ll}
\rho_{\varepsilon} \boldsymbol{\phi}_{\varepsilon} \mathbb{1}_{\Omega \backslash B_{\varepsilon}} \longrightarrow \rho \mathbb{1}_{Y \backslash A}(y) \boldsymbol{w}_{0}, & \rho_{\varepsilon} \frac{\partial^{m} \boldsymbol{\phi}_{\varepsilon}}{\partial t^{m}} \mathbb{1}_{\Omega \backslash B_{\varepsilon}} \longrightarrow \rho \mathbb{1}_{Y \backslash A}(y) \frac{\partial^{m} \boldsymbol{w}_{0}}{\partial t^{m}}, \\
\rho_{\varepsilon} \boldsymbol{\phi}_{\varepsilon}(0) \mathbb{1}_{\Omega \backslash B_{\varepsilon}} \longrightarrow \rho \mathbb{1}_{Y \backslash A}(y) \boldsymbol{w}_{0}(0), & \rho_{\varepsilon} \frac{\partial \boldsymbol{\phi}_{\varepsilon}}{\partial t}(0) \mathbb{1}_{\Omega \backslash B_{\varepsilon}} \longrightarrow \rho \mathbb{1}_{Y \backslash A}(y) \frac{\partial \boldsymbol{w}_{0}}{\partial t}(0) .
\end{array}
$$

By multiplying (3.5) by $\phi_{\varepsilon}$, after integrations by parts we obtain (see (6.3))

$$
\begin{aligned}
\int_{\Omega \times(0, T)} \rho_{\varepsilon} \boldsymbol{u}_{\varepsilon} \cdot \frac{\partial^{2} \phi_{\varepsilon}}{\partial t^{2}} d x d t+\int_{\Omega} \rho_{\varepsilon} \boldsymbol{a}_{0} \cdot \frac{\partial \phi_{\varepsilon}}{\partial t}(0) d x-\int_{\Omega} \rho_{\varepsilon} \boldsymbol{b}_{0} \cdot \phi_{\varepsilon}(0) d x \\
+\int_{\Omega \times(0, T)} \boldsymbol{e}\left(\boldsymbol{u}_{\varepsilon}\right): \boldsymbol{\sigma}_{\varepsilon}\left(\phi_{\varepsilon}\right) d x d t=\int_{\Omega \times(0, T)} \rho_{\varepsilon} \boldsymbol{f} \cdot \boldsymbol{\phi}_{\varepsilon} d x d t .
\end{aligned}
$$


By (3.7) and (3.39), we have

$$
\begin{aligned}
\int_{\Omega \times(0, T)} \rho_{\varepsilon} \boldsymbol{u}_{\varepsilon} \cdot \frac{\partial^{2} \phi_{\varepsilon}}{\partial t^{2}} d x d t=\int_{\Omega \times(0, T)} \rho \mathbb{1}_{\Omega \backslash B_{\varepsilon}} \boldsymbol{u}_{\varepsilon} \cdot \frac{\partial^{2} \phi_{\varepsilon}}{\partial t^{2}} d x d t \\
+\int_{\Omega \times(0, T)} \bar{\rho}_{1} \boldsymbol{u}_{\varepsilon} \cdot \frac{\partial^{2} \boldsymbol{\phi}_{\varepsilon}}{\partial t^{2}} d m_{\varepsilon}(x) d t .
\end{aligned}
$$

We deduce from (5.48) (see Corollary 5.6), and (6.15) that

$$
\lim _{\varepsilon \rightarrow 0} \int_{\Omega \times(0, T)} \rho_{\varepsilon} \mathbb{1}_{\Omega \backslash B_{\varepsilon}} \boldsymbol{u}_{\varepsilon} \cdot \frac{\partial^{2} \boldsymbol{\phi}_{\varepsilon}}{\partial t^{2}} d x d t=\int_{\Omega \times(0, T) \times Y} \rho \mathbb{1}_{Y \backslash A}(y) \boldsymbol{u}_{0} \cdot \frac{\partial^{2} \boldsymbol{w}_{0}}{\partial t^{2}} d x d t d y .
$$

By (5.11), and (6.13), we have

$$
\lim _{\varepsilon \rightarrow 0} \int_{\Omega \times(0, T)} \bar{\rho}_{1} \boldsymbol{u}_{\varepsilon} \cdot \frac{\partial^{2} \phi_{\varepsilon}}{\partial t^{2}} d m_{\varepsilon}(x) d t=\int_{\Omega \times(0, T)} \bar{\rho}_{1} \boldsymbol{v} \cdot \frac{\partial^{2} \boldsymbol{\psi}}{\partial t^{2}} d x d t .
$$

The last three equations imply

$$
\lim _{\varepsilon \rightarrow 0} \int_{\Omega \times(0, T)} \rho_{\varepsilon} \boldsymbol{u}_{\varepsilon} \cdot \frac{\partial^{2} \phi_{\varepsilon}}{\partial t^{2}} d x d t=\int_{\Omega \times(0, T) \times Y \backslash A} \underset{\rho \boldsymbol{u}_{0}}{ } \cdot \frac{\partial^{2} \boldsymbol{w}_{0}}{\partial t^{2}} d x d t d y+\int_{\Omega \times(0, T)} \bar{\rho}_{1} \boldsymbol{v} \cdot \frac{\partial^{2} \boldsymbol{\psi}}{\partial t^{2}} d x d t
$$

As, by (3.5), $\boldsymbol{a}_{0}, \boldsymbol{b}_{0}$, and $\boldsymbol{f}$ are continuous, we obtain by the same argument

$$
\begin{aligned}
& \lim _{\varepsilon \rightarrow 0} \int_{\Omega} \rho_{\varepsilon} \boldsymbol{a}_{0} \cdot \frac{\partial \boldsymbol{\phi}_{\varepsilon}}{\partial t}(0) d x=\int_{\Omega \times Y \backslash A} \rho \boldsymbol{a}_{0} \cdot \frac{\partial \boldsymbol{w}_{0}}{\partial t}(0) d x d y+\int_{\Omega} \bar{\rho}_{1} \boldsymbol{a}_{0} \cdot \frac{\partial^{2} \boldsymbol{\psi}}{\partial t^{2}}(0) d x, \\
& \lim _{\varepsilon \rightarrow 0} \int_{\Omega} \rho_{\varepsilon} \boldsymbol{b}_{0} \cdot \phi_{\varepsilon}(0) d x=\int_{\Omega \times Y \backslash A} \rho \boldsymbol{b}_{0} \cdot \boldsymbol{w}_{0}(0) d x d y+\int_{\Omega} \bar{\rho}_{1} \boldsymbol{b}_{0} \cdot \boldsymbol{\psi}(0) d x, \\
& \lim _{\varepsilon \rightarrow 0} \int_{\Omega \times(0, T)} \rho_{\varepsilon} \boldsymbol{f} \cdot \boldsymbol{\phi}_{\varepsilon} d x d t=\int_{\Omega \times(0, T) \times Y \backslash A} \underset{f}{ } \cdot \boldsymbol{w}_{0} d x d t d y+\int_{\Omega \times(0, T)} \bar{\rho}_{1} \boldsymbol{f} \cdot \frac{\partial^{2} \boldsymbol{\psi}}{\partial t^{2}} d x d t .
\end{aligned}
$$

We split the $4^{\text {th }}$ term on the left-hand side of (6.16) into the sum of three terms:

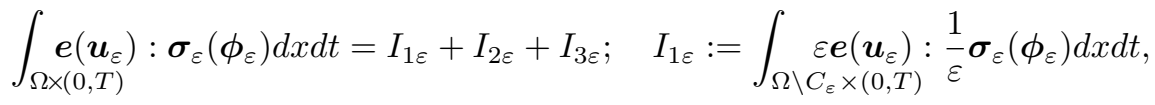

$$
\begin{aligned}
& I_{2 \varepsilon}:=\int_{C_{\varepsilon} \backslash B_{\varepsilon} \times(0, T)} \varepsilon \boldsymbol{e}\left(\boldsymbol{u}_{\varepsilon}\right): \frac{1}{\varepsilon} \boldsymbol{\sigma}_{\varepsilon}\left(\phi_{\varepsilon}\right) d x d t, \quad I_{3 \varepsilon}:=\int_{B_{\varepsilon} \times(0, T)}^{\boldsymbol{e}\left(\boldsymbol{u}_{\varepsilon}\right): \boldsymbol{\sigma}_{\varepsilon}\left(\phi_{\varepsilon}\right) d x d t .}
\end{aligned}
$$

By (6.10) and (6.11), we have $\boldsymbol{\phi}_{\varepsilon} \mathbb{1}_{\Omega \backslash C_{\varepsilon}}=\boldsymbol{w}_{0}\left(x, t, \frac{x}{\varepsilon}\right) \mathbb{1}_{\Omega \backslash C_{\varepsilon}}$. Taking (3.5), (3.6), and (3.34) into account, we deduce that

$$
\left|\frac{1}{\varepsilon} \boldsymbol{\sigma}_{\varepsilon}\left(\phi_{\varepsilon}\right)-\boldsymbol{\sigma}_{0 y}\left(\boldsymbol{w}_{0}\right)\left(x, t, \frac{x}{\varepsilon}\right)\right| \mathbb{1}_{\Omega \backslash C_{\varepsilon}} \leq C \varepsilon,
$$

where the operator $\boldsymbol{\sigma}_{0 y}$ is defined by (3.42). The following convergence 


$$
\mathbb{1}_{\Omega \backslash C_{\varepsilon}} \longrightarrow \mathbb{1}_{Y \backslash A},
$$

follows from (4.4) and from the strong convergence of $\mathbb{1}_{C_{\varepsilon} \backslash B_{\varepsilon}}$ to 0 in $L^{2}(\Omega)$, which results from (6.6) and (6.8). By applying Assertion (4.5) of Lemma 4.1 to $h_{0}:=$ $\boldsymbol{\sigma}_{0 y}\left(\boldsymbol{w}_{0}\right)$ and $\chi_{\varepsilon}:=\mathbb{1}_{\Omega \backslash C_{\varepsilon}}$, taking (6.21) into account, we infer

$$
\frac{1}{\varepsilon} \boldsymbol{\sigma}_{\varepsilon}\left(\phi_{\varepsilon}\right) \mathbb{1}_{\Omega \backslash C_{\varepsilon}} \longrightarrow \boldsymbol{\sigma}_{0 y}\left(\boldsymbol{w}_{0}\right) \mathbb{1}_{Y \backslash A}(y) .
$$

We deduce from (5.48), (6.20), and (6.23) that

$$
\lim _{\varepsilon \rightarrow 0} I_{1 \varepsilon}=\int_{\Omega \times(0, T) \times Y \backslash A} \boldsymbol{e}_{y}\left(\boldsymbol{u}_{0}\right): \boldsymbol{\sigma}_{0 y}\left(\boldsymbol{w}_{0}\right) d x d t d y .
$$

By (3.34), (6.8) and (6.14), we have $\int_{C_{\varepsilon} \backslash B_{\varepsilon} \times(0, T)}\left|\frac{1}{\varepsilon} \boldsymbol{\sigma}_{\varepsilon}\left(\phi_{\varepsilon}\right)\right|^{2} d x d t \leq C\left(\alpha_{\varepsilon}+\frac{\varepsilon^{2}}{\alpha_{\varepsilon}}\right)$ if $\vartheta>0$, and $\int_{C_{\varepsilon} \backslash B_{\varepsilon} \times(0, T)}\left|\frac{1}{\varepsilon} \sigma_{\varepsilon}\left(\phi_{\varepsilon}\right)\right|^{2} d x d t \leq C \frac{r_{\varepsilon}}{\alpha_{\varepsilon} \varepsilon}$ if $\vartheta=0$. Therefore, by (6.6), the sequence $\left(\frac{1}{\varepsilon} \boldsymbol{\sigma}_{\varepsilon}\left(\phi_{\varepsilon}\right) \mathbb{1}_{C_{\varepsilon} \backslash B_{\varepsilon}}\right)$ strongly converges to 0 in $L^{2}\left(\Omega \times(0, T) ; \mathbb{S}^{3}\right)$. Accordingly, we infer from (5.47) and (6.20) that

$$
\lim _{\varepsilon \rightarrow 0} I_{2 \varepsilon}=0
$$

Finally, the limit of the sequence $\left(I_{3 \varepsilon}\right)$ defined by (6.20) is computed in Lemma 7.1 in terms of $k$ and $\kappa$. Passing to the limit as $\varepsilon \rightarrow 0$ in (6.16), collecting (6.4), (6.18), (6.19), (6.20), (6.24), (6.25), and (7.5), we obtain the variational formulation given, according to the order of magnitude of $k$ and $\kappa$, by (6.26), (6.39), (6.41), or (6.32). We distinguish 4 cases:

Case $0<k<+\infty$. We find

$$
\begin{aligned}
& \int_{\Omega \times(0, T) \times Y \backslash A} \rho \boldsymbol{u}_{0} \cdot \frac{\partial^{2} \boldsymbol{w}_{0}}{\partial t^{2}} d x d t d y+\int_{\Omega \times(0, T) \times Y \backslash A} \rho \boldsymbol{a}_{0} \cdot \frac{\partial \boldsymbol{w}_{0}}{\partial t}(0)-\rho \boldsymbol{b}_{0} \cdot \boldsymbol{w}_{0}(0) d x d y \\
& +\int_{\Omega \times(0, T)} \bar{\rho}_{1} \boldsymbol{v} \cdot \frac{\partial^{2} \boldsymbol{\psi}}{\partial t^{2}} d x d t+\int_{\Omega} \bar{\rho}_{1} \boldsymbol{a}_{0} \cdot \frac{\partial \boldsymbol{\psi}}{\partial t}(0)-\bar{\rho}_{1} \boldsymbol{b}_{0} \cdot \boldsymbol{\psi}(0) d x \\
& +\int_{\Omega \times(0, T) \times Y \backslash A} \boldsymbol{e}_{y}\left(\boldsymbol{u}_{0}\right): \boldsymbol{\sigma}_{0 y}\left(\boldsymbol{w}_{0}\right) d x d t d y+k \int_{\Omega \times(0, T)} \boldsymbol{e}_{x^{\prime}}\left(\boldsymbol{v}^{\prime}\right): \boldsymbol{\sigma}_{x^{\prime}}\left(\boldsymbol{\psi}^{\prime}\right) d x d t \\
& =\int_{\Omega \times(0, T) \times Y \backslash A} \rho \boldsymbol{f} \cdot \boldsymbol{w}_{0} d x d t d y+\int_{\Omega \times(0, T)} \bar{\rho}_{1} \boldsymbol{f} \cdot \boldsymbol{\psi} d x d t,
\end{aligned}
$$

for all $\left(\boldsymbol{w}_{0}, \boldsymbol{\psi}\right) \in L^{2}(0, T ; H)$ satisfying (6.2)-(6.5). We set (see (6.1)) 


$$
\begin{aligned}
& \xi=\left(\boldsymbol{u}_{0}, \boldsymbol{v}\right), \quad \xi_{0}=\left(\boldsymbol{a}_{0}, \boldsymbol{a}_{0}\right), \quad \xi_{1}=\left(\boldsymbol{b}_{0}, \boldsymbol{b}_{0}\right), \quad h=(\boldsymbol{f}, \boldsymbol{f}), \\
& V:=\left\{\begin{array}{l|l}
\left(\boldsymbol{w}_{0}, \boldsymbol{\psi}\right) \in H \mid \begin{array}{l}
\boldsymbol{w}_{0} \in L^{2}\left(\Omega ; H_{\sharp}^{1}\left(Y ; \mathbb{R}^{3}\right)\right) \\
\psi_{1}, \psi_{2} \in L^{2}\left(0, L ; H_{0}^{1}\left(\Omega^{\prime}\right)\right)
\end{array}
\end{array}\right\} \quad \text { if } \vartheta>0, \\
& V:=\left\{\begin{array}{l|l}
\left(\boldsymbol{w}_{0}, \boldsymbol{\psi}\right) \in H & \begin{array}{l}
\boldsymbol{w}_{0} \in L^{2}\left(\Omega ; H_{\sharp}^{1}\left(Y ; \mathbb{R}^{3}\right)\right), \\
\psi_{1}, \psi_{2} \in L^{2}\left(0, L ; H_{0}^{1}\left(\Omega^{\prime}\right)\right) \\
\boldsymbol{w}_{0}^{\prime}(x, y)=\boldsymbol{\psi}^{\prime}(x) \text { on } \Omega \times \Sigma \\
\int_{\Sigma} w_{03}(., y) d \mathcal{H}^{2}(y)=\psi_{3}
\end{array}
\end{array}\right\} \quad \text { if } r_{\varepsilon} \ll \varepsilon, \\
& \bar{a}(\boldsymbol{v}, \boldsymbol{\psi}):=k \int_{\Omega} \boldsymbol{e}_{x^{\prime}}\left(\boldsymbol{v}^{\prime}\right): \boldsymbol{\sigma}_{x^{\prime}}\left(\boldsymbol{\psi}^{\prime}\right) d x, \\
& a\left(\left(\boldsymbol{u}_{0}, \boldsymbol{v}\right),\left(\boldsymbol{w}_{0}, \boldsymbol{\psi}\right)\right):=\int_{\Omega \times Y \backslash A} \boldsymbol{e}_{y}\left(\boldsymbol{u}_{0}\right): \boldsymbol{\sigma}_{y}\left(\boldsymbol{w}_{0}\right) d x d y+\bar{a}(\boldsymbol{v}, \boldsymbol{\psi}), \\
& \left(\left(\boldsymbol{u}_{0}, \boldsymbol{v}\right),\left(\boldsymbol{w}_{0}, \boldsymbol{\psi}\right)\right)_{V}:=\left(\left(\boldsymbol{u}_{0}, \boldsymbol{v}\right),\left(\boldsymbol{w}_{0}, \boldsymbol{\psi}\right)\right)_{H}+\bar{a}(\boldsymbol{v}, \boldsymbol{\psi})+\int_{\Omega \times Y \backslash A} \underset{\nabla_{y}}{\boldsymbol{u}_{0}} \cdot \nabla_{y} \boldsymbol{w}_{0} d x d y .
\end{aligned}
$$

By (4.8), (5.11) and (5.48), we have $\xi=\left(\boldsymbol{u}_{0}, \boldsymbol{v}\right) \in L^{2}(0, T ; V), \frac{\partial \xi}{\partial t} \in L^{2}(0, T ; H)$, thus by a density argument the variational formulation (6.26) is equivalent to (4.23). By (6.1), (6.27), and the following Korn's inequality (see [42], p. 14),

$$
\int_{Y \backslash A}|\boldsymbol{w}|^{2}+|\nabla(\boldsymbol{w})|^{2} d y \leq C \int_{Y \backslash A}|\boldsymbol{w}|^{2}+|\boldsymbol{e}(\boldsymbol{w})|^{2} d y \quad \forall \boldsymbol{w} \in H^{1}\left(Y \backslash A ; \mathbb{R}^{3}\right),
$$

for all $\widetilde{\xi}=\left(\boldsymbol{w}_{0}, \boldsymbol{\psi}\right) \in V$, the following holds

$$
\begin{aligned}
\|\widetilde{\xi}\|_{V}^{2} & =|\widetilde{\xi}|_{H}^{2}+\bar{a}(\psi, \psi)+\left\|\nabla_{y}\left(\boldsymbol{w}_{0}\right)\right\|_{L^{2}\left(\Omega \times Y \backslash A ; \mathbb{R}^{3}\right)}^{2} \\
& \leq C|\widetilde{\xi}|_{H}^{2}+C|| \boldsymbol{e}_{y}\left(\boldsymbol{w}_{0}\right) \|_{L^{2}\left(\Omega \times Y \backslash A ; \mathbb{R}^{3}\right)}^{2}+\bar{a}(\psi, \psi) \leq C|\widetilde{\xi}|_{H}^{2}+C a(\widetilde{\xi}, \widetilde{\xi}),
\end{aligned}
$$

yielding (4.18). We deduce from Theorem 4.4 that $\xi=\left(\boldsymbol{u}_{0}, \boldsymbol{v}\right)$ is the unique solution to (6.26). By (4.19), (4.20), (6.27), the following holds

$$
\xi \in C([0, T] ; V) \cap C^{1}([0, T] ; H), \xi(0)=\left(\boldsymbol{a}_{0}, \boldsymbol{a}_{0}\right), \frac{\partial \xi}{\partial t}(0)=\left(\boldsymbol{b}_{0}, \boldsymbol{b}_{0}\right) .
$$

It follows from (6.29), from the following inequalities (deduced from (6.1), (6.27))

$$
\begin{aligned}
&\left\|\boldsymbol{w}_{0}\right\|_{L^{2}\left(\Omega ; H_{\sharp}^{1}\left(Y ; \mathbb{R}^{3}\right)\right)}+\|\boldsymbol{\psi}\|_{L^{2}\left(\Omega ; \mathbb{R}^{3}\right)}+\left\|\psi_{1}\right\|_{L^{2}\left(0, L ; H_{0}^{1}\left(\Omega^{\prime}\right)\right)} \\
&+\left\|\psi_{2}\right\|_{L^{2}\left(0, L ; H_{0}^{1}\left(\Omega^{\prime}\right)\right)} \leq C\left\|\left(\boldsymbol{w}_{0}, \boldsymbol{\psi}\right)\right\|_{V} \forall\left(\boldsymbol{w}_{0}, \boldsymbol{\psi}\right) \in V, \\
&\left\|\boldsymbol{w}_{0}\right\|_{L^{2}\left(\Omega \times Y ; \mathbb{R}^{3}\right)}+\|\boldsymbol{\psi}\|_{L^{2}\left(\Omega ; \mathbb{R}^{3}\right)} \leq C\left|\left(\boldsymbol{w}_{0}, \boldsymbol{\psi}\right)\right|_{H} \quad \forall\left(\boldsymbol{w}_{0}, \boldsymbol{\psi}\right) \in H,
\end{aligned}
$$

and the following implication, holding for any couple $\left(E_{1}, E_{2}\right)$ of Banach spaces

$$
\left.\begin{array}{l}
A \in \mathcal{L}\left(E_{1}, E_{2}\right) \\
B \in C^{k}\left([0, T] ; E_{1}\right)
\end{array}\right\} \Rightarrow\left[A \circ B \in C^{k}\left([0, T] ; E_{2}\right) ; \quad \frac{d^{s}}{d t^{s}}(A \circ B)=A \circ \frac{d^{s}}{d t^{s}} B \forall s \leq k\right],
$$


applied with $B=\xi=\left(\boldsymbol{u}_{0}, \boldsymbol{v}\right), E_{1} \in\{H, V\},\left(A(\xi), E_{2}\right) \in\left\{\left(\boldsymbol{u}_{0}, L^{2}\left(\Omega ; H_{\sharp}^{1}\left(Y ; \mathbb{R}^{3}\right)\right)\right)\right.$, $\left.\left(\boldsymbol{v}, L^{2}\left(\Omega ; \mathbb{R}^{3}\right)\right),\left(v_{\alpha}, L^{2}\left(0, L ; H_{0}^{1}\left(\Omega^{\prime}\right)\right)\right),\left(\boldsymbol{u}_{0}, L^{2}\left(\Omega \times Y ; \mathbb{R}^{3}\right)\right),\left(\boldsymbol{v}, L^{2}(\Omega)\right)\right\}$, that

$$
\begin{aligned}
& \boldsymbol{u}_{0} \in C\left([0, T] ; L^{2}\left(\Omega ; H_{\sharp}^{1}\left(Y ; \mathbb{R}^{3}\right)\right)\right) \cap C^{1}\left([0, T] ; L^{2}\left(\Omega \times Y ; \mathbb{R}^{3}\right)\right), \\
& \boldsymbol{u}_{0}(0)=\boldsymbol{a}_{0}, \frac{\partial \boldsymbol{u}_{0}}{\partial t}(0)=\boldsymbol{b}_{0}, \\
& \boldsymbol{v} \in C^{1}\left([0, T] ; L^{2}\left(\Omega ; \mathbb{R}^{3}\right)\right), \boldsymbol{v}(0)=\boldsymbol{a}_{0}, \frac{\partial \boldsymbol{v}}{\partial t}(0)=\boldsymbol{b}_{0}, \\
& v_{1}, v_{2} \in C\left([0, T] ; L^{2}\left(0, L ; H_{0}^{1}\left(\Omega^{\prime}\right)\right)\right) \cap C^{1}\left([0, T] ; L^{2}(\Omega)\right) .
\end{aligned}
$$

Next we prove that the variational problem (6.26) is equivalent to (3.40). Setting $\boldsymbol{\psi}=0$ in (6.26), noticing that $\boldsymbol{e}_{y}\left(\boldsymbol{u}_{0}\right): \boldsymbol{\sigma}_{0 y}\left(\boldsymbol{w}_{0}\right)=\boldsymbol{\sigma}_{0 y}\left(\boldsymbol{u}_{0}\right): \nabla_{y}\left(\boldsymbol{w}_{0}\right)$, we get

$$
\begin{aligned}
& \int_{\Omega \times(0, T) \times Y \backslash A}^{\rho \boldsymbol{u}_{0}} \cdot \frac{\partial^{2} \boldsymbol{w}_{0}}{\partial t^{2}} d x d t d y+\int_{\Omega \times Y \backslash A}^{\rho \boldsymbol{a}_{0}} \cdot \frac{\partial \boldsymbol{w}_{0}}{\partial t}(0) d x d y-\int_{\Omega \times Y \backslash A}^{\rho \boldsymbol{b}_{0}} \cdot \boldsymbol{w}_{0}(0) d x d y
\end{aligned}
$$

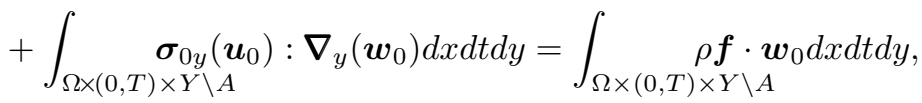

and, letting $\boldsymbol{w}_{0}$ vary over $\mathcal{D}\left(\Omega \times(0, T) \times Y \backslash A ; \mathbb{R}^{3}\right)$, deduce

$$
\rho \frac{\partial^{2} \boldsymbol{u}_{0}}{\partial t^{2}}-\operatorname{div}_{y}\left(\boldsymbol{\sigma}_{0 y}\left(\boldsymbol{u}_{0}\right)\right)=\rho \boldsymbol{f} \quad \text { in } \Omega \times(0, T) \times Y \backslash A .
$$

By integrating (6.32) by parts with respect to $(t, y)$ for an arbitrary $\boldsymbol{w}_{0}$ satisfying (6.2), (6.3), (6.5), we infer from (6.33) that $\int_{\Omega \times(0, T) \times \partial Y} \boldsymbol{\sigma}_{0 y}\left(\boldsymbol{u}_{0}\right) \boldsymbol{\nu} \cdot \boldsymbol{w}_{0} d x d t d \mathcal{H}^{2}(y)=0$ $(\boldsymbol{\nu}:=$ outward pointing normal to $\partial Y)$. Noticing that $\boldsymbol{\sigma}_{0 y}\left(\boldsymbol{u}_{0}\right) \boldsymbol{\nu}=0 \mathcal{H}^{2}$ a. e. on $\partial Y \cap \bar{A}$ (because if $\vartheta>0$, then $A=B$ and, by (5.49), $\boldsymbol{\sigma}_{0 y}\left(\boldsymbol{u}_{0}\right)=0$ in $B$, whereas if $r_{\varepsilon} \ll \varepsilon$, then $A=\Sigma$ and $\left.\mathcal{H}^{2}(\partial Y \cap \bar{\Sigma})=0\right)$, we deduce

$$
\boldsymbol{\sigma}_{0 y}\left(\boldsymbol{u}_{0}\right) \boldsymbol{\nu}(x, t, y)=-\boldsymbol{\sigma}_{0 y}\left(\boldsymbol{u}_{0}\right) \boldsymbol{\nu}(x, t,-y) \quad \text { on } \quad \Omega \times(0, T) \times \partial Y .
$$

Fixing $\left(\boldsymbol{w}_{0}, \boldsymbol{\psi}\right) \in L^{2}(0, T ; H)$ satisfying (6.2), (6.3), we infer from the $Y$-periodicity of $\boldsymbol{w}_{0},(6.5)$, and (6.34), that (see (3.42))

$$
\begin{aligned}
& -\int_{\Omega \times(0, T) \times \partial(Y \backslash A)} \boldsymbol{\sigma}_{0 y}\left(\boldsymbol{u}_{0}\right) \boldsymbol{\nu} \cdot \boldsymbol{w}_{0} d x d t d \mathcal{H}^{2}(y)=-\int_{\Omega \times(0, T) \times \partial(Y \backslash A) \cap \bar{A}} \boldsymbol{\sigma}_{0 y}\left(\boldsymbol{u}_{0}\right) \boldsymbol{\nu}_{Y \backslash A} \cdot \boldsymbol{\psi} d x d t d \mathcal{H}^{2}(y) \\
& =\int_{\Omega \times(0, T)} \boldsymbol{g}\left(\boldsymbol{u}_{0}\right) \cdot \boldsymbol{\psi} d x d t .
\end{aligned}
$$

By multiplying (6.33) by $\boldsymbol{w}_{0}$ and by integrating it by parts over $\Omega \times(0, T) \times Y \backslash A$, thanks to (6.31), (6.34), (6.35) we obtain

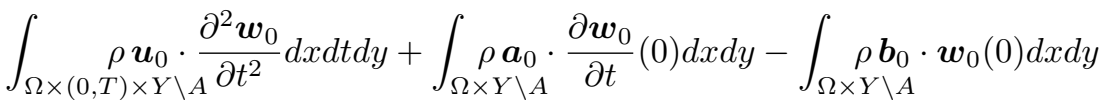

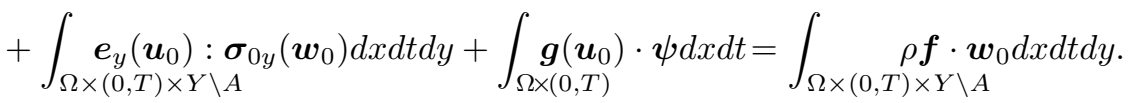

By subtracting (6.36) from (6.26), we find 


$$
\begin{aligned}
\int_{\Omega \times(0, T)} \bar{\rho}_{1} \boldsymbol{v} & \cdot \frac{\partial^{2} \boldsymbol{\psi}}{\partial t^{2}} d x d t-\int_{\Omega \times(0, T)} \boldsymbol{g}\left(\boldsymbol{u}_{0}\right) \cdot \boldsymbol{\psi} d x d t+k \int_{\Omega \times(0, T)} \boldsymbol{e}_{x^{\prime}}\left(\boldsymbol{v}^{\prime}\right): \boldsymbol{\sigma}_{x^{\prime}}\left(\boldsymbol{\psi}^{\prime}\right) d x d t \\
& +\int_{\Omega} \bar{\rho}_{1} \boldsymbol{a}_{0} \cdot \frac{\partial \boldsymbol{\psi}}{\partial t}(0) d x-\int_{\Omega} \bar{\rho}_{1} \boldsymbol{b}_{0} \cdot \boldsymbol{\psi}(0) d x=\int_{\Omega \times(0, T)} \bar{\rho}_{1} \boldsymbol{f} \cdot \boldsymbol{\psi} d x d t
\end{aligned}
$$

Making $\boldsymbol{\psi}$ vary in $\mathcal{D}\left(\Omega \times(0, T) ; \mathbb{R}^{3}\right)$, we infer

$$
\bar{\rho}_{1} \frac{\partial^{2} \boldsymbol{v}}{\partial t^{2}}-k \operatorname{div} \boldsymbol{\sigma}_{x^{\prime}}\left(\boldsymbol{v}^{\prime}\right)=\bar{\rho}_{1} \boldsymbol{f}+\boldsymbol{g}\left(\boldsymbol{u}_{0}\right) \quad \text { in } \quad \Omega \times(0, T) .
$$

By $(6.31),(6.33),(6.34),(6.38)$, and Lemma 5.5 , the couple $\left(\boldsymbol{u}_{0}, \boldsymbol{v}\right)$ is a solution to (3.40), (3.45). Conversely, any solution to (3.40), (3.45) satisfies (6.26).

Case $k=+\infty, \kappa=0$. We obtain

$$
\begin{aligned}
& \int_{\Omega \times(0, T) \times Y \backslash A} \rho \boldsymbol{u}_{0} \cdot \frac{\partial^{2} \boldsymbol{w}_{0}}{\partial t^{2}} d x d t d y+\int_{\Omega \times Y \backslash A} \rho \boldsymbol{a}_{0} \cdot \frac{\partial \boldsymbol{w}_{0}}{\partial t}(0)-\rho \boldsymbol{b}_{0} \cdot \boldsymbol{w}_{0}(0) d x d y \\
& +\int_{\Omega \times(0, T)} \bar{\rho}_{1} v_{3} \frac{\partial^{2} \psi_{3}}{\partial t^{2}} d x d t+\int_{\Omega} \bar{\rho}_{1} a_{03} \frac{\partial \psi_{3}}{\partial t}(0)-\bar{\rho}_{1} b_{03} \psi_{03}(0) d x
\end{aligned}
$$

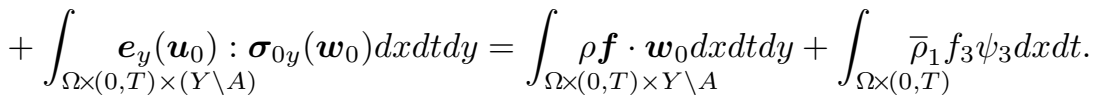

This variational formulation is satisfied for all $\left(\boldsymbol{w}_{0}, \boldsymbol{\psi}\right) \in L^{2}(0, T ; H)$ verifying (6.2)(6.5). We set ( $H$ and $V$ being given by (6.1), (6.27))

$$
\begin{aligned}
& \xi=\left(\boldsymbol{u}_{0}, \boldsymbol{v}\right), \quad H^{(2)}:=\left\{\left(\boldsymbol{w}_{0}, \boldsymbol{\psi} \in H, \psi_{1}=\psi_{2}=0\right\},\right. \\
& (., .)_{H^{(2)}}:=(., .)_{H}, \quad V^{(2)}:=V \cap H^{(2)}, \quad(.,)_{V^{(2)}}:=(., .)_{V}, \\
& h^{(2)}:=\left(\boldsymbol{f} \mathbb{1}_{Y \backslash A}+f_{3} \boldsymbol{e}_{3} \mathbb{1}_{A}, f_{3} \boldsymbol{e}_{3}\right), \\
& a^{(2)}\left(\left(\boldsymbol{u}_{0}, \boldsymbol{v}\right),\left(\boldsymbol{w}_{0}, \boldsymbol{\psi}\right)\right):=\int_{\Omega \times Y \backslash A} \boldsymbol{e}_{y}\left(\boldsymbol{u}_{0}\right): \boldsymbol{\sigma}_{y}\left(\boldsymbol{w}_{0}\right) d x d y, \\
& \xi_{0}^{(2)}:=\left(\boldsymbol{a}_{0} \mathbb{1}_{Y \backslash A}+a_{03} \boldsymbol{e}_{3} \mathbb{1}_{A}, a_{03} \boldsymbol{e}_{3}\right), \quad \xi_{1}^{(2)}:=\left(\boldsymbol{b}_{0} \mathbb{1}_{Y \backslash A}+b_{03} \boldsymbol{e}_{3} \mathbb{1}_{A}, b_{03} \boldsymbol{e}_{3}\right) .
\end{aligned}
$$

By (4.8), (5.10), (5.48) and (5.80), we have $\xi \in L^{2}\left(0, T ; V^{(2)}\right)$ and $\xi^{\prime} \in L^{2}\left(0, T ; H^{(2)}\right)$. Therefore, by a density argument, the variational problem (6.39) is equivalent to (4.23). By (6.28), (6.40), the estimate (4.18) is satisfied. We deduce from Theorem 4.4 that $\xi=\left(\boldsymbol{u}_{0}, \boldsymbol{v}\right)$ is the unique solution to (6.39) and that $\xi \in C\left([0, T] ; V^{(2)}\right) \cap$ $C^{1}\left([0, T] ; H^{(2)}\right), \xi(0)=\xi_{0}^{(2)}, \frac{\partial \xi}{\partial t}(0)=\xi_{1}^{(2)}$. Then, repeating the argument employed to prove (6.31), we infer from (6.30) and (6.40) that the initial-boundary conditions and regularity properties stated in (3.44), (3.46) are satisfied. Setting $\psi_{3}=0$ in (6.39), we get (6.32) and deduce (6.33), (6.34), (6.35), (6.36). Then, subtracting (6.36) from (6.39), taking (6.4) into account, we find

$$
\begin{array}{r}
\int_{\Omega \times(0, T)} \bar{\rho}_{1} v_{3} \frac{\partial^{2} \psi_{3}}{\partial t^{2}} d x d t-\int_{\Omega \times(0, T)}\left(\boldsymbol{g}\left(\boldsymbol{u}_{0}\right)\right)_{3} \psi_{3} d x d t+\int_{\Omega} \bar{\rho}_{1} a_{03} \frac{\partial \psi_{3}}{\partial t}(0) d x \\
-\int_{\Omega} \bar{\rho}_{1} b_{03} \psi_{3}(0) d x=\int_{\Omega \times(0, T)} \bar{\rho}_{1} f_{3} \psi_{3} d x d t
\end{array}
$$


Making $\psi_{3}$ vary in $\mathcal{D}(\Omega \times(0, T))$, we deduce that $\bar{\rho}_{1} \frac{\partial^{2} v_{3}}{\partial t^{2}}=\bar{\rho}_{1} f_{3}+\left(\boldsymbol{g}\left(\boldsymbol{u}_{0}\right)\right)_{3}$ in $\Omega \times(0, T)$ and infer that $\left(\boldsymbol{u}_{0}, \boldsymbol{v}\right)$ is solution to (3.40), (3.46).

Case $0<\kappa<+\infty$. Passing to the limit as $\varepsilon \rightarrow 0$ in (6.16), we obtain

$$
\begin{aligned}
& \int_{\Omega \times(0, T) \times Y \backslash A} \rho \boldsymbol{u}_{0} \cdot \frac{\partial^{2} \boldsymbol{w}_{0}}{\partial t^{2}} d x d t d y+\int_{\Omega \times Y \backslash A} \rho \boldsymbol{a}_{0} \cdot \frac{\partial \boldsymbol{w}_{0}}{\partial t}(0)-\rho \boldsymbol{b}_{0} \cdot \boldsymbol{w}_{0}(0) d x d y \\
& +\int_{\Omega \times(0, T)} \bar{\rho}_{1} v_{3} \frac{\partial^{2} \psi_{3}}{\partial t^{2}} d x d t+\int_{\Omega} \bar{\rho}_{1} a_{03} \frac{\partial \psi_{3}}{\partial t}(0)-\bar{\rho}_{1} b_{03} \psi_{03}(0) d x \\
& +\int_{\Omega \times(0, T) \times(Y \backslash A)} \boldsymbol{e}_{y}\left(\boldsymbol{u}_{0}\right): \boldsymbol{\sigma}_{0 y}\left(\boldsymbol{w}_{0}\right) d x d t d y+\frac{\kappa}{6} \int_{\Omega \times(0, T)} \boldsymbol{H}\left(v_{3}\right): \boldsymbol{H}^{\boldsymbol{\sigma}}\left(\psi_{3}\right) d x d t \\
& =\int_{\Omega \times(0, T) \times Y \backslash A} \rho \boldsymbol{f} \cdot \boldsymbol{w}_{0} d x d t d y+\int_{\Omega \times(0, T)} \bar{\rho}_{1} f_{3} \psi_{3} d x d t,
\end{aligned}
$$

for all $\left(\boldsymbol{w}_{0}, \boldsymbol{\psi}\right) \in L^{2}(0, T ; H)$ verifying (6.2)-(6.5). We set (see (6.27), (6.40), (7.6))

$$
\begin{aligned}
& H^{(3)}:=H^{(2)}, \\
& V^{(3)}:=\left\{\begin{array}{l|l}
\left(\boldsymbol{w}_{0}, \psi_{3} \boldsymbol{e}_{3}\right) \in V^{(2)} & \begin{array}{l}
\psi_{3} \in L^{2}\left(0, L ; H_{0}^{2}\left(\Omega^{\prime}\right)\right) \\
\boldsymbol{w}_{0}(x, y)=\psi_{3}(x) \boldsymbol{e}_{3} \text { on } \Omega \times \Sigma
\end{array}
\end{array},\right. \\
& \left(\left(\left(\boldsymbol{u}_{0}, v_{3} \boldsymbol{e}_{3}\right),\left(\boldsymbol{w}_{0}, \psi_{3} \boldsymbol{e}_{3}\right)\right)\right)_{V^{(3)}}:=\left(\left(\left(\boldsymbol{u}_{0}, v_{3} \boldsymbol{e}_{3}\right),\left(\boldsymbol{w}_{0}, \psi_{3} \boldsymbol{e}_{3}\right)\right)\right)_{V} \\
& +\int_{\Omega}\left(\frac{\partial^{2} v_{3}}{\partial x_{1}^{2}} \frac{\partial^{2} \psi_{3}}{\partial x_{1}^{2}}+\frac{\partial^{2} v_{3}}{\partial x_{2}^{2}} \frac{\partial^{2} \psi_{3}}{\partial x_{2}^{2}}+\frac{\partial^{2} v_{3}}{\partial x_{1}^{2}} \frac{\partial^{2} \psi_{3}}{\partial x_{2}^{2}}+\frac{\partial^{2} v_{3}}{\partial x_{2}^{2}} \frac{\partial^{2} \psi_{3}}{\partial x_{1}^{2}}\right) d x \\
& \left.\bar{a}^{(3)}\left(v_{3} \boldsymbol{e}_{3}, \psi_{3} \boldsymbol{e}_{3}\right)\right):=\frac{\kappa}{6} \int_{\Omega} \boldsymbol{H}\left(v_{3}\right): \boldsymbol{H}^{\boldsymbol{\sigma}}\left(\psi_{3}\right) d x d t, \\
& a^{(3)}\left(\left(\boldsymbol{u}_{0}, \boldsymbol{v}\right),\left(\boldsymbol{w}_{0}, \boldsymbol{\psi}\right)\right):=\int_{\Omega \times(Y \backslash B)} \boldsymbol{e}_{y}\left(\boldsymbol{u}_{0}\right): \boldsymbol{\sigma}_{y}\left(\boldsymbol{w}_{0}\right) d x d y+\bar{a}^{(3)}(\boldsymbol{v}, \boldsymbol{\psi}), \\
& \xi_{0}^{(3)}:=\xi_{0}^{(2)}, \quad \xi_{1}^{(3)}:=\xi_{1}^{(2)}, \quad h^{(3)}:=h^{(2)} .
\end{aligned}
$$

By Corollary 5.6 (ii) and assertions (4.8), (5.10), (5.48), and (5.80), there holds $\xi=$ $\left(\boldsymbol{u}_{0}, \boldsymbol{v}\right) \in L^{2}\left(0, T ; V^{(3)}\right)$ and $\frac{\partial \xi}{\partial t} \in L^{2}\left(0, T ; H^{(3)}\right)$ hence, by a density argument, the variational formulation (6.41) is equivalent to (4.23). By (6.27), (6.28), (6.40), (6.42), (6.43), and (7.6), for all $\widetilde{\xi}=\left(\boldsymbol{w}_{0}, \boldsymbol{\psi}\right) \in V^{(3)}$, we have

$$
\begin{aligned}
\|\widetilde{\xi}\|_{V^{(3)}}^{2} & \leq\|\widetilde{\xi}\|_{V}^{2}+C \bar{a}^{(3)}(\boldsymbol{\psi}, \boldsymbol{\psi}) \leq C\left(|\widetilde{\xi}|_{H}+a(\widetilde{\xi}, \widetilde{\xi})+\bar{a}^{(3)}(\boldsymbol{\psi}, \boldsymbol{\psi})\right) \\
& \leq C\left(|\widetilde{\xi}|_{H^{(3)}}+a^{(3)}(\widetilde{\xi}, \widetilde{\xi})\right),
\end{aligned}
$$

hence Assumption (4.18) is satisfied. We deduce from Theorem 4.4 that $\xi=\left(\boldsymbol{u}_{0}, \boldsymbol{v}\right)$ is the unique solution to (6.41) and that $\xi \in C\left([0, T] ; V^{(3)}\right) \cap C^{1}\left([0, T] ; H^{(3)}\right), \xi(0)=\xi_{0}^{(3)}$, $\frac{\partial \xi}{\partial t}(0)=\xi_{1}^{(3)}$, yielding, by the inequality $(6.30)$ joined with

$$
\left\|\psi_{3}\right\|_{L^{2}\left(0, L ; H_{0}^{2}\left(\Omega^{\prime}\right)\right)} \leq C\left\|\left(\boldsymbol{w}_{0}, \boldsymbol{\psi}\right)\right\|_{V^{(3)}}, \forall\left(\boldsymbol{w}_{0}, \boldsymbol{\psi}\right) \in V^{(3)},
$$

the initial-boundary conditions and regularity properties stated in (3.44), (3.47). Repeating the argument for the case $0<k<+\infty$, we set $\psi_{3}=0$ in (6.41), obtain (6.32), deduce (6.33), (6.34), (6.35), (6.36), subtract (6.36) from (6.41), and get 


$$
\begin{aligned}
& \int_{\Omega \times(0, T)} \bar{\rho}_{1} v_{3} \frac{\partial^{2} \psi_{3}}{\partial t^{2}} d x d t+\frac{\kappa}{6} \int_{\Omega \times(0, T)} \boldsymbol{H}\left(v_{3}\right): \boldsymbol{H}^{\boldsymbol{\sigma}}\left(\psi_{3}\right) d x d t \\
& \quad-\int_{\Omega \times(0, T)}\left(\boldsymbol{g}\left(\boldsymbol{u}_{0}\right)\right)_{3} \boldsymbol{\psi}_{3} d x d t-\int_{\Omega} \bar{\rho}_{1}\left(\boldsymbol{b}_{0}\right)_{3} \psi_{3}(x, 0) d x=\int_{\Omega \times(0, T)} \bar{\rho}_{1} f_{3} \psi_{3} d x d t .
\end{aligned}
$$

By (7.6), the following equation holds in the sense of distributions in $\mathcal{D}^{\prime}(\Omega \times(0, T))$

$$
\frac{\kappa}{6}\left\langle\boldsymbol{H}\left(v_{3}\right): \boldsymbol{H}^{\boldsymbol{\sigma}}\left(\psi_{3}\right)\right\rangle_{\mathcal{D}^{\prime}, \mathcal{D}}=\frac{\kappa}{3} \frac{l+1}{l+2}\left\langle\sum_{\alpha, \beta=1}^{2} \frac{\partial^{4} v_{3}}{\partial x_{\alpha}^{2} \partial x_{\beta}^{2}}, \psi_{3}\right\rangle_{\mathcal{D}^{\prime}, \mathcal{D}} .
$$

Making $\psi_{3}$ vary in $\mathcal{D}(\Omega \times(0, T))$ in (6.44), we infer

$$
\bar{\rho}_{1} \frac{\partial^{2} v_{3}}{\partial t^{2}}+\frac{\kappa}{3} \frac{l+1}{l+2} \sum_{\alpha, \beta=1}^{2} \frac{\partial^{4} v_{3}}{\partial x_{\alpha}^{2} \partial x_{\beta}^{2}}=\bar{\rho}_{1} f_{3}+\left(\boldsymbol{g}\left(\boldsymbol{u}_{0}\right)\right)_{3}, \quad \text { in } \Omega \times(0, T),
$$

and deduce that $\left(\boldsymbol{u}_{0}, \boldsymbol{v}\right)$ satisfies (3.40), (3.47).

Case $\kappa=+\infty$. By (7.5) we have $I_{3 \varepsilon}=0$. By passing to the limit as $\varepsilon \rightarrow 0$ in (6.16), we obtain (6.32) and, taking (5.80) into account, deduce in a similar manner that $\left(\boldsymbol{u}_{0}, \boldsymbol{v}\right)$ satisfies (3.40), (3.48). The proof of Theorem 3.12 is achieved.

6.2. Proofs of theorems 3.1 and 3.7. Under the assumptions of Theorem 3.1, by (3.12) and (5.5), the sequence $\left(\boldsymbol{u}_{\varepsilon}\right)$ (resp. $\left.\left(\frac{\partial \boldsymbol{u}_{\varepsilon}}{\partial t}\right)\right)$ is bounded in $L^{\infty}\left(0, T ; H_{0}^{1}\left(\Omega ; \mathbb{R}^{3}\right)\right)$ (resp. $L^{\infty}\left(0, T ; L^{2}\left(\Omega ; \mathbb{R}^{3}\right)\right)$ ), therefore by the Aubin-Lions-Simon lemma (see [51, Corollary 6$]),\left(\boldsymbol{u}_{\varepsilon}\right)$ strongly converges in $L^{2}\left(0, T ; L^{2}\left(\Omega ; \mathbb{R}^{3}\right)\right)$ and weakly* converges in $L^{\infty}\left(0, T ; H_{0}^{1}\left(\Omega ; \mathbb{R}^{3}\right)\right)$, up to a subsequence, to some $\boldsymbol{u} \in L^{\infty}\left(0, T ; H_{0}^{1}\left(\Omega ; \mathbb{R}^{3}\right)\right)$. In particular, assumption (5.14) of Lemma 5.3 is satisfied, hence $n \boldsymbol{u}=n \boldsymbol{v}$.

Under the assumptions of Theorem 3.7, by the apriori estimates (5.5), the sequence $\left(\boldsymbol{u}_{\varepsilon}\right)$ is bounded in $L^{\infty}\left(0, T ; L^{2}\left(\Omega ; \mathbb{R}^{3}\right)\right)$, hence weakly* converges in $L^{\infty}(0, T$; $\left.L^{2}\left(\Omega ; \mathbb{R}^{3}\right)\right)$, up to a subsequence, to some $\boldsymbol{u} \in L^{\infty}\left(0, T ; L^{2}\left(\Omega ; \mathbb{R}^{3}\right)\right)$. By (3.26) and (3.27), Assumption (5.13) of Lemma 5.3 is satisfied, thus we also get $n \boldsymbol{u}=n \boldsymbol{v}$. Applying Corollary 5.6, we deduce in both cases from (5.11), (5.31), (5.80), and (7.5), that

$$
\begin{array}{ll}
\boldsymbol{u}_{\varepsilon} m_{\varepsilon} \stackrel{\star}{*} n \boldsymbol{u} & \text { weakly* in } L^{\infty}\left(0, T ; \mathcal{M}\left(\bar{\Omega} ; \mathbb{R}^{3}\right)\right), \\
u_{1}, u_{2} \in L^{\infty}\left(0, T ; L_{n}^{2}\left(0, L ; H_{0}^{1}\left(\Omega^{\prime}\right)\right)\right), & \\
\boldsymbol{e}_{x^{\prime}}\left(\boldsymbol{u}_{\varepsilon}^{\prime}\right) m_{\varepsilon} \stackrel{\star}{*} \boldsymbol{e}_{x^{\prime}}\left(\boldsymbol{u}^{\prime}\right) & \text { weakly* in } L^{\infty}\left(0, T ; \mathcal{M}\left(\bar{\Omega} ; \mathbb{S}^{3}\right)\right), \\
n u_{1}=n u_{2}=0, & \text { if } k=+\infty, \\
u_{3} \in L^{\infty}\left(0, T ; L_{n}^{2}\left(0, L ; H_{0}^{2}\left(\Omega^{\prime}\right)\right)\right) & \text { if } \kappa>0, \\
n \boldsymbol{u}=0 & \text { if } \kappa=+\infty, \\
\mathcal{I}_{n, k, \kappa}(\boldsymbol{v}, \boldsymbol{\psi})=\mathcal{I}_{n, k, \kappa}(\boldsymbol{u}, \boldsymbol{\psi}) . &
\end{array}
$$

Let us check that

$$
\mathbb{1}_{\Omega \backslash B_{\varepsilon}} \stackrel{\star}{*} 1-\vartheta n \text { weakly* in } L^{\infty}(\Omega),
$$


where $\vartheta$ is defined by (3.10). If $\vartheta=0,(6.46)$ follows from the fact that $\left|B_{\varepsilon}\right| \rightarrow 0$. Otherwise, if $\vartheta>0$, then the sequence $\left(\frac{\varepsilon}{r_{\varepsilon}} \mathbb{1}_{B_{\varepsilon}}\right)$ is bounded in $L^{\infty}(\Omega)$ and, by (4.7), weakly* converges in $L^{\infty}(\Omega)$ to $n$. It then follows from (3.10) that $\left(\mathbb{1}_{B_{\varepsilon}}\right)$ weakly* converges in $L^{\infty}(\Omega)$ to $\vartheta n$, yielding (6.46). Next, we check that

$$
\boldsymbol{u}_{\varepsilon} \mathbb{1}_{\Omega \backslash B_{\varepsilon}} \rightarrow \boldsymbol{u}(1-\vartheta n) \quad \text { weakly in } L^{2}\left(\Omega \times(0, T) ; \mathbb{R}^{3}\right) .
$$

If $\vartheta=0$, Assertion (6.47) follows from the weak convergence of $\left(\boldsymbol{u}_{\varepsilon}\right)$ to $\boldsymbol{u}$ in $L^{2}(\Omega \times$ $(0, T))$ and the convergence of $\mathcal{L}^{3}\left(B_{\varepsilon}\right)$ to 0 . Otherwise, if $\vartheta>0$, then $\left(\frac{\varepsilon}{r_{\varepsilon}} \boldsymbol{u}_{\varepsilon} \mathbb{1}_{B_{\varepsilon}}\right)$ is bounded in $L^{2}(\Omega \times(0, T))$, and weakly converges, by (6.45), to $n \boldsymbol{u}$. Hence, by (3.10), $\left(\boldsymbol{u}_{\varepsilon} \mathbb{1}_{B_{\varepsilon}}\right)$ weakly converges to $n \vartheta \boldsymbol{u}$, yielding (6.47).

We fix a field $\boldsymbol{\psi}$ verifying (6.2), (6.3), and

$$
n \psi_{1}=n \psi_{2}=0 \quad \text { if } \quad k=+\infty ; \quad n \boldsymbol{\psi}=0 \quad \text { if } \quad \kappa=+\infty .
$$

The sequence of test fields $\left(\phi_{\varepsilon}\right)$ is defined by substituting $\boldsymbol{\psi}$ for $\boldsymbol{w}_{0}$ in (6.11), that is

$$
\boldsymbol{\phi}_{\varepsilon}(x, t):=\eta_{\varepsilon}(x) \widehat{\boldsymbol{\psi}}_{\varepsilon}(x, t)+\left(1-\eta_{\varepsilon}(x)\right) \boldsymbol{\psi}(x, t),
$$

where $\widehat{\boldsymbol{\psi}}_{\varepsilon}(x, t)$ is described in Section 7 , and $\eta_{\varepsilon}$ satisfies (6.10), now with respect to the non-periodic sets $B_{\varepsilon}, C_{\varepsilon}$ given by (3.4), (6.7). We assume that (see Remark 6.1)

$$
\begin{gathered}
\frac{r_{\varepsilon}}{\varepsilon} \ll \alpha_{\varepsilon} \ll 1 \quad \text { under the assumptions of Theorem 3.1, } \\
\mu_{0 \varepsilon} \ll \alpha_{\varepsilon} \ll 1 \quad \text { under the assumptions of Theorem 3.7. }
\end{gathered}
$$

By (6.10), (6.49), and (7.4), the following estimates hold in $\Omega \times(0, T)$ for $m \in\{1,2\}$ :

$$
\left|\phi_{\varepsilon}(x, t)-\boldsymbol{\psi}\left(x, t, \frac{x}{\varepsilon}\right)\right|+\left|\frac{\partial^{m} \boldsymbol{\phi}_{\varepsilon}}{\partial t^{m}}(x, t)-\frac{\partial^{m} \boldsymbol{\psi}}{\partial t^{m}}\left(x, t, \frac{x}{\varepsilon}\right)\right| \leq C r_{\varepsilon} .
$$

We deduce from (6.8) and from the estimate $\boldsymbol{\sigma}_{\varepsilon}\left(\phi_{\varepsilon}(x, t)\right) \leq C \frac{\mu_{0 \varepsilon}}{\alpha_{\varepsilon}}$ in $C_{\varepsilon} \backslash B_{\varepsilon} \times(0, T)$, obtained in a similar manner as (6.14), that

$$
\int_{C_{\varepsilon} \backslash B_{\varepsilon} \times(0, T)}\left|\boldsymbol{\sigma}_{\varepsilon}\left(\phi_{\varepsilon}(x, t)\right)\right|^{2} d x d t \leq C \frac{\mu_{\varepsilon 0}^{2} r_{\varepsilon}}{\alpha_{\varepsilon} \varepsilon} .
$$

We multiply Equation (3.5) by $\phi_{\varepsilon}$ and integrate it by parts to get (see (6.16), (6.20))

$$
\begin{gathered}
\int_{\Omega \times(0, T)} \rho_{\varepsilon} \boldsymbol{u}_{\varepsilon} \cdot \frac{\partial^{2} \phi_{\varepsilon}}{\partial t^{2}} d x d t+\int_{\Omega} \rho_{\varepsilon} \boldsymbol{a}_{0} \cdot \frac{\partial \phi_{\varepsilon}}{\partial t}(0) d x-\int_{\Omega} \rho_{\varepsilon} \boldsymbol{b}_{0} \cdot \phi_{\varepsilon}(0) d x \\
+I_{1 \varepsilon}+I_{2 \varepsilon}+I_{3 \varepsilon}=\int_{\Omega \times(0, T)} \rho_{\varepsilon} \boldsymbol{f} \cdot \boldsymbol{\phi}_{\varepsilon} d x d t
\end{gathered}
$$

By the same argument as the one used to get (6.18), (6.19), splitting each term as in (6.17) and taking into account (3.7), (4.7), (6.46), (6.47), (6.51), we obtain 


$$
\begin{aligned}
& \lim _{\varepsilon \rightarrow 0} \int_{\Omega \times(0, T)} \rho_{\varepsilon} \boldsymbol{u}_{\varepsilon} \cdot \frac{\partial^{2} \boldsymbol{\phi}_{\varepsilon}}{\partial t^{2}} d x d t=\int_{\Omega \times(0, T)} \rho(1-\vartheta n) \boldsymbol{u} \cdot \frac{\partial^{2} \boldsymbol{\psi}}{\partial t^{2}} d x d t+\int_{\Omega \times(0, T)} \bar{\rho}_{1} \boldsymbol{u} \cdot \frac{\partial^{2} \boldsymbol{\psi}}{\partial t^{2}} n d x d t, \\
& \lim _{\varepsilon \rightarrow 0} \int_{\Omega} \rho_{\varepsilon} \boldsymbol{a}_{0} \cdot \frac{\partial \boldsymbol{\phi}_{\varepsilon}}{\partial t}(0) d x=\int_{\Omega} \rho(1-\vartheta n) \boldsymbol{a}_{0} \cdot \frac{\partial \boldsymbol{\psi}}{\partial t}(0) d x+\int_{\bar{\rho}_{1}} \boldsymbol{a}_{0} \cdot \frac{\partial^{2} \boldsymbol{\psi}}{\partial t^{2}}(0) n d x d t \\
& \lim _{\varepsilon \rightarrow 0} \int_{\Omega} \rho_{\varepsilon} \boldsymbol{b}_{0} \cdot \boldsymbol{\phi}_{\varepsilon}(0) d x=\int_{\Omega} \rho(1-\vartheta n) \boldsymbol{b}_{0} \cdot \boldsymbol{\psi}(0) d x+\int_{\Omega} \bar{\rho}_{1} \boldsymbol{b}_{0} \cdot \boldsymbol{\psi}(0) n d x d t, \\
& \lim _{\varepsilon \rightarrow 0} \int_{\Omega \times(0, T)} \rho_{\varepsilon} \boldsymbol{f} \cdot \boldsymbol{\phi}_{\varepsilon} d x d t=\int_{\Omega \times(0, T)} \rho(1-\vartheta n) \boldsymbol{f} \cdot \boldsymbol{\psi} d x d t+\int_{\Omega \times(0, T)} \bar{\rho}_{1} \boldsymbol{f} \cdot \frac{\partial^{2} \boldsymbol{\psi}}{\partial t^{2}} n d x d t .
\end{aligned}
$$

Under the assumptions of Theorem 3.1, by (3.12), (3.13) and (6.49) we have $\boldsymbol{\sigma}_{\varepsilon}\left(\boldsymbol{\phi}_{\varepsilon}\right)=$ $\boldsymbol{\sigma}(\boldsymbol{\psi})$ in $\Omega \backslash C_{\varepsilon} \times(0, T)$, and by (3.11) and (6.7), $\lim _{\varepsilon \rightarrow 0}\left|C_{\varepsilon}\right|=0$, therefore the sequence $\left(\boldsymbol{\sigma}_{\varepsilon}\left(\phi_{\varepsilon}\right) \mathbb{1}_{\Omega \backslash C_{\varepsilon}}\right)$ strongly converges to $\boldsymbol{\sigma}(\boldsymbol{\psi})$ in $\left.L^{2}\left(\Omega \times(0, T) ; \mathbb{S}^{3}\right)\right)$. We deduce from the weak ${ }^{*}$ convergence of $\left(\boldsymbol{u}_{\varepsilon}\right)$ to $\boldsymbol{u}$ in $L^{\infty}\left(0, T ; H_{0}^{1}\left(\Omega ; \mathbb{R}^{3}\right)\right)$ that

$$
\lim _{\varepsilon \rightarrow 0} I_{1 \varepsilon}=\int_{\Omega \times(0, T)} \boldsymbol{e}(\boldsymbol{u}): \boldsymbol{\sigma}(\boldsymbol{\psi}) d x d t
$$

Under the assumptions of Theorem 3.7, noticing that $\left|\boldsymbol{\sigma}_{\varepsilon}\left(\boldsymbol{\phi}_{\varepsilon}\right) \mathbb{1}_{\Omega \backslash C_{\varepsilon}}\right|=\left|\boldsymbol{\sigma}_{\varepsilon}(\boldsymbol{\psi}) \mathbb{1}_{\Omega \backslash C_{\varepsilon}}\right| \leq$ $C \mu_{0 \varepsilon}$ and taking (3.26), (5.5), (6.20) into account, we get

$$
\limsup _{\varepsilon \rightarrow 0} I_{1 \varepsilon} \leq \limsup _{\varepsilon \rightarrow 0} C \mu_{0 \varepsilon}^{\frac{1}{2}}\left(\int_{\Omega \times(0, T)} \mu_{0 \varepsilon}\left|\boldsymbol{e}\left(\boldsymbol{u}_{\varepsilon}\right)(\tau)\right|^{2} d x d t\right)^{\frac{1}{2}}=0 .
$$

By (5.5), and (6.52), we have

$I_{2 \varepsilon} \leq\left(\int_{\Omega \times(0, T)}\left|\boldsymbol{e}\left(\boldsymbol{u}_{\varepsilon}\right)\right|^{2} d x d t\right)^{\frac{1}{2}}\left(\int_{C_{\varepsilon} \backslash B_{\varepsilon} \times(0, T)}\left|\boldsymbol{\sigma}_{\varepsilon}\left(\boldsymbol{\phi}_{\varepsilon}(x, t)\right)\right|^{2} d x d t\right)^{\frac{1}{2}} \leq C\left(\frac{\mu_{0 \varepsilon}}{\alpha_{\varepsilon}} \frac{r_{\varepsilon}}{\varepsilon}\right)^{\frac{1}{2}}$.

Under the assumptions of Theorem 3.1 (resp. Theorem 3.7), we deduce from (3.12) and (6.50) that

$$
\lim _{\varepsilon \rightarrow 0} I_{2 \varepsilon}=0 .
$$

Collecting (6.54), (6.55), (6.56), (6.57), and (7.5), by passing to the limit as $\varepsilon \rightarrow 0$ in (6.16), we obtain, under the assumptions of Theorem 3.1,

$$
\begin{aligned}
& \int_{\Omega \times(0, T)}\left(\rho+\bar{\rho}_{1} n\right) \boldsymbol{u} \cdot \frac{\partial^{2} \boldsymbol{\psi}}{\partial t^{2}} d x d t+\int_{\Omega \times(0, T)}\left(\rho+\bar{\rho}_{1} n\right)\left(\boldsymbol{a}_{0} \cdot \frac{\partial \boldsymbol{\psi}}{\partial t}(0)-\boldsymbol{b}_{0} \cdot \boldsymbol{\psi}(0)\right) d x \\
& +\int_{\Omega \times(0, T)} \boldsymbol{e}(\boldsymbol{u}): \boldsymbol{\sigma}(\boldsymbol{\psi}) d x d t+\mathcal{I}_{n, k, \kappa}(\boldsymbol{u}, \boldsymbol{\psi})=\int_{\Omega \times(0, T)}\left(\rho+\bar{\rho}_{1} n\right) \boldsymbol{f} \cdot \boldsymbol{u} d x d t
\end{aligned}
$$

and, under the assumptions of Theorem 3.7, 


$$
\begin{aligned}
& \int_{\Omega \times(0, T)}\left(\rho(1-\vartheta n)+\bar{\rho}_{1} n\right) \boldsymbol{u} \cdot \frac{\partial^{2} \boldsymbol{\psi}}{\partial t^{2}} d x d t \\
&+\int_{\Omega \times(0, T)}\left(\rho(1-\vartheta n)+\bar{\rho}_{1} n\right)\left(\boldsymbol{a}_{0} \cdot \frac{\partial \boldsymbol{\psi}}{\partial t}(0)-\boldsymbol{b}_{0} \cdot \boldsymbol{\psi}(0)\right) d x+\mathcal{I}_{n, k, \kappa}(\boldsymbol{u}, \boldsymbol{\psi}) \\
& \quad=\int_{\Omega \times(0, T)}\left(\rho(1-\vartheta n)+\bar{\rho}_{1} n\right) \boldsymbol{f} \cdot \boldsymbol{u} d x d t
\end{aligned}
$$

The variational formulation (6.59), joined with (6.45), is equivalent to (4.23), where

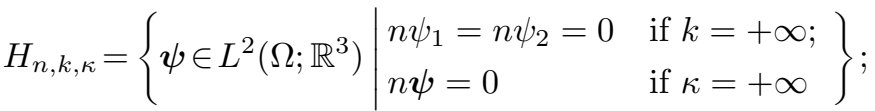

$$
\begin{aligned}
& V_{n, k, \kappa}=\left\{\begin{array}{l|l}
\psi \in H_{n, k, \kappa} & \begin{array}{l}
\psi_{1}, \psi_{2} \in L_{n}^{2}\left(0, L ; H_{0}^{1}\left(\Omega^{\prime}\right)\right) \\
\psi_{3} \in L_{n}^{2}\left(0, L ; H_{0}^{2}\left(\Omega^{\prime}\right)\right)
\end{array} \text { if } 0<k \\
\text { if } 0<\kappa,
\end{array}\right\} \\
& (\boldsymbol{u}, \boldsymbol{\psi})_{H_{n, k, \kappa}}=\int_{\Omega}\left(\rho(1-\vartheta n)+\bar{\rho}_{1} n\right) \boldsymbol{u} \cdot \boldsymbol{\psi} d x \\
& ((\boldsymbol{u}, \boldsymbol{\psi}))_{V_{n, k, \kappa}}=(\boldsymbol{u}, \boldsymbol{\psi})_{H_{n, k, \kappa}}+\mathcal{I}_{n, k, \kappa}(\boldsymbol{u}, \boldsymbol{\psi}) ; \quad a_{n, k, \kappa}(\boldsymbol{u}, \boldsymbol{\psi})=\mathcal{I}_{n, k, \kappa}(\boldsymbol{u}, \boldsymbol{\psi}) ; \\
& h_{n, k, \kappa}=\mathcal{H}_{n, k, \kappa}(\boldsymbol{f}), \xi_{0, n, k, \kappa}=\mathcal{H}_{n, k, \kappa}\left(\boldsymbol{a}_{0}\right), \xi_{1, n, k, \kappa}=\mathcal{H}_{n, k, \kappa}\left(\boldsymbol{b}_{0}\right) \text {, } \\
& \mathcal{H}_{n, k, \kappa}(\boldsymbol{g}):= \begin{cases}\boldsymbol{g} & \text { if } 0<k<+\infty, \\
\left(g_{1} \mathbb{1}_{\{n=0\}}, g_{2} \mathbb{1}_{\{n=0\}}, g_{3}\right), & \text { if } k=+\infty, \kappa<+\infty, \\
\boldsymbol{g} \mathbb{1}_{\{n=0\}} & \text { if } \kappa=+\infty .\end{cases}
\end{aligned}
$$

The variational formulation (6.58), joined with (6.45), is equivalent to (4.23), with data deduced from (6.60), (6.61) by substituting $\widetilde{V}_{n, k, \kappa}$ and $\widetilde{a}_{n, k, \kappa}$ for $V_{n, k, \kappa}$ and $a_{n, k, \kappa}$, where

$$
\begin{aligned}
& \widetilde{V}_{n, k, \kappa}:=V_{n, k, \kappa} \cap H_{0}^{1}\left(\Omega ; \mathbb{R}^{3}\right) ;((\boldsymbol{u}, \boldsymbol{\psi}))_{\widetilde{V}_{n, k, \kappa}}=((\boldsymbol{u}, \boldsymbol{\psi}))_{V_{n, k, \kappa}}+\int_{\Omega} \boldsymbol{\nabla} \boldsymbol{u} \cdot \nabla \boldsymbol{\psi} d x, \\
& \widetilde{a}_{n, k, \kappa}(\boldsymbol{u}, \boldsymbol{\psi}):=a_{n, k, \kappa}(\boldsymbol{u}, \boldsymbol{\psi})+\int_{\Omega} \boldsymbol{e}(\boldsymbol{u}): \boldsymbol{\sigma}(\boldsymbol{\psi}) d x
\end{aligned}
$$

The assumptions of Theorem 4.4 are satisfied in both cases, guaranteeing existence, uniqueness and regularity properties of the solution. Finally, by integrations by parts, it is easy to check that the variational problems (6.58), (6.59), associated with (6.45), are equivalent, under (3.17), to the problems announced in theorems 3.1, 3.7.

REMARK 6.1. The assumption stated in the first line of (6.50) is employed to derive (6.25) and requires (3.11). The case $\mu_{0 \varepsilon}=\mu>0, \vartheta>0, k=+\infty$ is open.

REMARK 6.2 (Multiphase case). Theorems 3.1, 3.7 can be extended to the case of $m$ distributions $B_{\varepsilon}^{[s]}(s \in\{1, . ., m\})$ of parallel disjoint homothetical layers of thickness $r_{\varepsilon}^{[s]}$, Lamé coefficients $\lambda_{1 \varepsilon}^{[s]}, \mu_{1 \varepsilon}^{[s]}$, and mass density $\frac{\varepsilon}{r_{\varepsilon}^{[s]}} \bar{\rho}_{1}^{[s]}$, defined in terms of a finite subset $\omega_{\varepsilon}^{[s]}$ of $(0, L)$ and $r_{\varepsilon}^{[s]}$ by a formula like (3.4). The sets $\omega_{\varepsilon}^{[s]}$ are disjoint and their union $\omega_{\varepsilon}:=\bigcup_{s=1}^{m} \omega_{\varepsilon}^{[s]}$ satisfies (3.2), which implies that the minimal distance between 
two distincts points of $\omega_{\varepsilon}$ is equal to $\varepsilon$. We suppose that $\varepsilon>r_{\varepsilon}^{[s]}(1+\delta), \forall s \in\{1, . ., m\}$ for some $\delta>0$ and set $\vartheta^{[s]}:=\lim _{\varepsilon \rightarrow 0} \frac{r_{\varepsilon}^{[s]}}{\varepsilon}$. The Lamé coefficients in $\Omega \backslash \bigcup_{s=1}^{m} B_{\varepsilon}^{[s]}$ are assume to be constant and denoted by $\lambda_{0 \varepsilon}, \mu_{0 \varepsilon}$.

When $\lambda_{0 \varepsilon}, \mu_{0 \varepsilon}$ satisfy (3.26) and each sequence $\left(n_{\varepsilon}^{[s]}\right)$ strongly converges to $n^{[s]}$ in $L^{1}(\Omega)$, the solution to (3.5) weakly* converges in $L^{\infty}\left(0, T ; L^{2}\left(\Omega ; \mathbb{R}^{3}\right)\right)$ to the unique solution to the problem (4.23), where the data are deduced from (6.60) as follows:

$$
\begin{aligned}
& H:=\bigcap_{s=1}^{m} H_{n^{[s]}, k^{[s]}, \kappa^{[s]}} ; \quad(\boldsymbol{u}, \boldsymbol{\psi})_{H}=\int_{\Omega}\left(\rho\left(1-\sum_{s=1}^{m} \vartheta^{[s]} n^{[s]}\right)+\bar{\rho}_{1}^{[s]} n^{[s]}\right) \boldsymbol{u} \cdot \boldsymbol{\psi} d x \\
& V:=\bigcap_{s=1}^{m} V_{n^{[s]}, k[s], \kappa[s]} ; \quad((\boldsymbol{u}, \boldsymbol{\psi}))_{V}=(\boldsymbol{u}, \boldsymbol{\psi})_{H}+\sum_{s=1}^{m} \mathcal{I}_{n^{[s]}, k^{[s]}, \kappa^{[s]}}(\boldsymbol{u}, \boldsymbol{\psi}), \\
& a(\boldsymbol{u}, \boldsymbol{\psi})=\sum_{s=1}^{m} a_{n^{[s]}, k^{[s]}, \kappa^{[s]}}(\boldsymbol{u}, \boldsymbol{\psi}), \\
& h=\mathcal{H}(\boldsymbol{f}), \xi_{0}=\mathcal{H}\left(\boldsymbol{a}_{0}\right), \xi_{1}=\mathcal{H}\left(\boldsymbol{b}_{0}\right), \\
& (\mathcal{H}(\boldsymbol{g})(x))_{\alpha}= \begin{cases}0 & \text { if } \exists s \in\{1, . ., m\}, n^{[s]}(x)>0 \text { and } k^{[s]}=+\infty, \\
g_{\alpha} & \text { otherwise, } \quad(\alpha \in\{1,2\}),\end{cases} \\
& (\mathcal{H}(\boldsymbol{g})(x))_{3}= \begin{cases}0 & \text { if } \exists s \in\{1, . ., m\}, n^{[s]}(x)>0 \text { and } \kappa^{[s]}=+\infty, \\
g_{3}(x) & \text { otherwise. }\end{cases}
\end{aligned}
$$

When $\lambda_{0 \varepsilon}, \mu_{0 \varepsilon}$ satisfy (3.12), and when $\vartheta^{[s]}=0$ for each $s \in\{1, . ., m\}$, the solution to (3.5) weakly* converges in $L^{\infty}\left(0, T ; H_{0}^{1}\left(\Omega ; \mathbb{R}^{3}\right)\right)$ to the unique solution to (4.23), with data $\widetilde{H}, \widetilde{V}, \widetilde{a} \ldots$ deduced from $H, V$, a... defined in (6.63) as follows:

$$
\begin{aligned}
& \widetilde{H}:=H ; \quad \widetilde{V}=V \cap H_{0}^{1}\left(\Omega ; \mathbb{R}^{3}\right) ; \quad((\boldsymbol{u}, \boldsymbol{\psi}))_{\widetilde{V}}:=((\boldsymbol{u}, \boldsymbol{\psi}))_{V}+\int_{\Omega} \boldsymbol{\nabla} \boldsymbol{u} \cdot \boldsymbol{\nabla} \boldsymbol{\psi} d x \\
& \widetilde{a}(\boldsymbol{u}, \boldsymbol{\psi})=a(\boldsymbol{u}, \boldsymbol{\psi})+\int_{\Omega} \boldsymbol{e}(\boldsymbol{u}): \boldsymbol{\sigma}(\boldsymbol{\psi}) d x ; \quad\left(\widetilde{h}, \widetilde{\xi}_{0}, \widetilde{\xi}_{1}\right):=\left(h, \xi_{0}, \xi_{1}\right) .
\end{aligned}
$$

REMark 6.3 (Elliptic case). When $\lambda_{0 \varepsilon}, \mu_{0 \varepsilon}$ satisfy (3.12), and when $\vartheta^{[s]}=0$ for each $s \in\{1, . ., m\}$, the solution $\boldsymbol{u}_{\varepsilon}$ to the equilibrium problem (3.32) is bounded in $H_{0}^{1}\left(\Omega ; \mathbb{R}^{3}\right)$ and weakly converges to the unique field $\boldsymbol{u} \in \widetilde{V}$ satisfying $\widetilde{a}(\boldsymbol{u}, \boldsymbol{\psi})=$ $(\boldsymbol{f}, \boldsymbol{\psi})_{\widetilde{H}}, \forall \boldsymbol{\psi} \in \widetilde{V}$, where $\widetilde{V}$ is the Hilbert space and $\widetilde{a}(.,$.$) the continuous coercive$ bilinear form on $\widetilde{V}$ given by (6.64).

If $\lambda_{0 \varepsilon}, \mu_{0 \varepsilon}$ satisfy (3.26), each sequence $\left(n_{\varepsilon}^{[s]}\right)$ strongly converges to $n^{[s]}$ in $L^{2}(\Omega)$, and $\boldsymbol{u}_{\varepsilon}$ is bounded in $L^{2}\left(\Omega ; \mathbb{R}^{3}\right)$, then $\boldsymbol{u}_{\varepsilon}$ weakly converges, up to a subsequence, to some $\boldsymbol{u} \in V$ verifying $a(\boldsymbol{u}, \boldsymbol{\psi})=(\boldsymbol{f}, \boldsymbol{\psi})_{H} \forall \boldsymbol{\psi} \in V$, with $H, V, a(.,$.$) defined by (6.63).$ In this case, the non-negative bilinear form a(., .) may fail to be coercive on $L^{2}\left(\Omega ; \mathbb{R}^{3}\right)$ and the sequence $\boldsymbol{u}_{\varepsilon}$ to be bounded in $L^{2}\left(\Omega ; \mathbb{R}^{3}\right)$. These coercivity and boundedness are guaranteed by the existence of $s \in\{1, . ., m\}$ and $c>0$ such that $\kappa^{[s]}>0$ and $n_{\varepsilon}^{[s]} \geq$ c a.e. in $\Omega_{\varepsilon}:=\bigcup_{i \in Z_{\varepsilon}^{[s]}}\left(\varepsilon i-\frac{\varepsilon}{2}, \varepsilon i+\frac{\varepsilon}{2}\right]$ (see (3.15)). (Notice that if the second assumption in (3.2) is replaced by $\min _{j, j^{\prime} \in J_{\varepsilon}, j \neq j^{\prime}}\left|\omega_{\varepsilon}^{j}-\omega_{\varepsilon}^{j^{\prime}}\right|=\eta \varepsilon$ for some arbitrarily fixed $\eta \in\left(0, \frac{1}{2}\right)$, our proofs are unchanged and $n_{\varepsilon}^{[s]} \geq c \mathbb{1}_{\Omega_{\varepsilon}}$ does not imply that $B_{\varepsilon}^{[s]}$ is $\varepsilon$-periodic). 
Sketch of the proof. Let $s$ be such that $\kappa^{[s]}>0$. The bilinear form associated with (3.32), namely $a_{\varepsilon}(\boldsymbol{\varphi}, \boldsymbol{\psi})=\int_{\Omega} \boldsymbol{e}(\boldsymbol{\varphi}): \boldsymbol{\sigma}_{\varepsilon}(\boldsymbol{\psi}) d x \forall(\boldsymbol{\varphi}, \boldsymbol{\psi}) \in\left(H_{0}^{1}\left(\Omega ; \mathbb{R}^{3}\right)\right)^{2}$, satisfies, by (3.5), (3.8), and (3.26)

$$
a_{\varepsilon}(\boldsymbol{\varphi}, \boldsymbol{\varphi}) \geq C \int_{\Omega} \varepsilon^{2}|\boldsymbol{e}(\boldsymbol{\varphi})|^{2} d x+C \int_{\Omega}\left|\frac{1}{r_{\varepsilon}^{[s]}} \boldsymbol{e}(\boldsymbol{\varphi})\right|^{2} d m_{\varepsilon}^{[s]} .
$$

Let $\boldsymbol{u}_{\varepsilon}$ be a sequence in $H_{0}^{1}\left(\Omega ; \mathbb{R}^{3}\right)$, and let $m_{\varepsilon}^{[s]}, \hat{\boldsymbol{v}}_{\varepsilon}^{[s]}, \overline{\boldsymbol{v}}_{\varepsilon}^{[s]}$ be defined by substituting $\omega_{\varepsilon}^{[s]}$ for $\omega_{\varepsilon}$ in (3.39), (5.17), (5.20). We have, since $n_{\varepsilon}^{[s]} \geq c \mathbb{1}_{\Omega_{\varepsilon}}$,

$$
\int_{\Omega}\left|\boldsymbol{u}_{\varepsilon}\right|^{2} d x \leq \int_{\Omega \backslash \Omega_{\varepsilon}}\left|\boldsymbol{u}_{\varepsilon}\right|^{2} d x+C \int_{\Omega_{\varepsilon}}\left|n_{\varepsilon}^{[s]} \boldsymbol{u}_{\varepsilon}-\overline{\boldsymbol{v}}_{\varepsilon}^{[s]}\right|^{2} d x+C \int_{\Omega}\left|\overline{\boldsymbol{v}}_{\varepsilon}^{[s]}\right|^{2} d x .
$$

Looking back at (5.28), and using the fact that $\boldsymbol{u}_{\varepsilon}$ vanishes on $\partial \Omega$, we obtain

$$
\begin{aligned}
\int_{\Omega \backslash \Omega_{\varepsilon}} & \left|\boldsymbol{u}_{\varepsilon}\right|^{2} d x+C \int_{\Omega_{\varepsilon}}\left|n_{\varepsilon}^{[s]} \boldsymbol{u}_{\varepsilon}-\overline{\boldsymbol{v}}_{\varepsilon}^{[s]}\right|^{2} d x \\
& \leq C \varepsilon^{2} \int_{\Omega \backslash \Omega_{\varepsilon}}\left|\frac{\partial \boldsymbol{u}_{\varepsilon}}{\partial x_{3}}\right|^{2}(\tau) d x+C \varepsilon^{2} \sum_{i \in Z_{\varepsilon}^{[s]}} \int_{\Omega^{\prime} \times\left(\varepsilon i-\frac{\varepsilon}{2}, \varepsilon i+\frac{\varepsilon}{2}\right]}\left|\frac{\partial \boldsymbol{u}_{\varepsilon}}{\partial x_{3}}\right|^{2}(\tau) d x \\
& \leq C \varepsilon^{2} \int_{\Omega}\left|\nabla \boldsymbol{u}_{\varepsilon}\right|^{2} d x \leq C \varepsilon^{2} \int_{\Omega}\left|\boldsymbol{e}\left(\boldsymbol{u}_{\varepsilon}\right)\right|^{2} d x
\end{aligned}
$$

yielding $\int_{\Omega}\left|\boldsymbol{u}_{\varepsilon}\right|^{2} d x \leq C \varepsilon^{2} \int_{\Omega}\left|\boldsymbol{e}\left(\boldsymbol{u}_{\varepsilon}\right)\right|^{2} d x+C \int_{\Omega}\left|\overline{\boldsymbol{v}}_{\varepsilon}^{[s]}\right|^{2} d x$. On the other hand, by (5.22), (5.18), (5.1),

$$
\begin{aligned}
\int_{\Omega}\left|\overline{\boldsymbol{v}}_{\varepsilon}^{[s]}\right|^{2} d x & =\int_{\Omega}\left|\hat{\boldsymbol{v}}_{\varepsilon}^{[s]}\right|^{2} d m_{\varepsilon}^{[s]} \leq C \int_{\Omega}\left|\boldsymbol{u}_{\varepsilon}-\hat{\boldsymbol{v}}_{\varepsilon}^{[s]}\right|^{2} d m_{\varepsilon}^{[s]}+C \int_{\Omega}\left|\boldsymbol{u}_{\varepsilon}\right|^{2} d m_{\varepsilon}^{[s]} \\
& \leq C \varepsilon r_{\varepsilon}^{[s]} \int_{\Omega}\left|\boldsymbol{e}\left(\boldsymbol{u}_{\varepsilon}\right)\right|^{2} d m_{\varepsilon}^{[s]}+C \int_{\Omega}\left|\frac{1}{r_{\varepsilon}^{[s]}} \boldsymbol{e}\left(\boldsymbol{u}_{\varepsilon}\right)\right|^{2} d m_{\varepsilon}^{[s]}
\end{aligned}
$$

therefore, for all $\boldsymbol{u}_{\varepsilon} \in H_{0}^{1}\left(\Omega ; \mathbb{R}^{3}\right), \int_{\Omega}\left|\boldsymbol{u}_{\varepsilon}\right|^{2} d x \leq C a_{\varepsilon}\left(\boldsymbol{u}_{\varepsilon}, \boldsymbol{u}_{\varepsilon}\right)$. In the particular case when $\boldsymbol{u}_{\varepsilon}$ is the solution to (3.32), we infer $\int_{\Omega}\left|\boldsymbol{u}_{\varepsilon}\right|^{2} d x \leq C \int_{\Omega} \boldsymbol{f} \cdot \boldsymbol{u}_{\varepsilon} d x \leq$ $C\left(\int_{\Omega}\left|\boldsymbol{u}_{\varepsilon}\right|^{2} d x\right)^{\frac{1}{2}}$, hence $\left(\boldsymbol{u}_{\varepsilon}\right)$ is bounded in $L^{2}\left(\Omega ; \mathbb{R}^{3}\right)$. We choose a smooth field $\psi \in V$ and consider the associated sequence of test field $\phi_{\varepsilon}$ used for the proof of the multiphase case, whose construction is similar to (6.49). Repeating the argument of [8, p. $40,($ iii $) \Rightarrow(i)]$, we find that $a(\boldsymbol{\psi}, \boldsymbol{\psi})=\lim _{\varepsilon \rightarrow 0} a_{\varepsilon}\left(\boldsymbol{\phi}_{\varepsilon}, \boldsymbol{\phi}_{\varepsilon}\right) \geq c \lim _{\varepsilon \rightarrow 0} \int_{\Omega}\left|\boldsymbol{\phi}_{\varepsilon}\right|^{2} d x=$ $\int_{\Omega}|\psi|^{2} d x$. By a density argument, we deduce $\int_{\Omega}|\psi|^{2} d x \leq C a(\boldsymbol{\psi}, \boldsymbol{\psi}) \forall \boldsymbol{\psi} \in V$.

7. Appendix. A common step in the proofs of theorems 3.1, 3.7, and 3.12 lies in the computation of the limit of the sequence $\left(I_{3 \varepsilon}\right)$ defined by (see $(6.11),(6.20)$ )

$$
I_{3 \varepsilon}:=\int_{B_{\varepsilon} \times(0, T)} \boldsymbol{e}\left(\boldsymbol{u}_{\varepsilon}\right): \boldsymbol{\sigma}_{\varepsilon}\left(\widehat{\boldsymbol{\psi}}_{\varepsilon}\right) d x d t
$$


where $\boldsymbol{u}_{\varepsilon}$ is the solution to (3.5) and the oscillating test fields $\widehat{\boldsymbol{\psi}}_{\varepsilon}$ is defined bellow, in terms of $\boldsymbol{\psi} \in C^{\infty}\left([0, T] ; \mathcal{D}\left(\Omega ; \mathbb{R}^{3}\right)\right)$ satisfying (6.4), of $\delta$ given by (3.3), and of the order of magnitude of the parameters $k$ and $\kappa$. We introduce the field $\bar{\psi}_{\varepsilon}$ given by

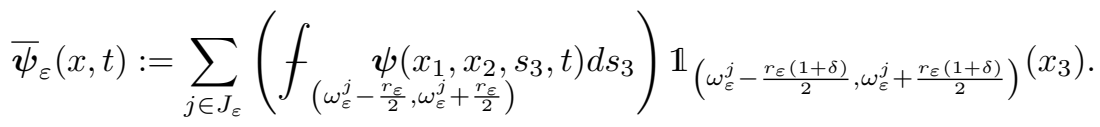

(i) If $0<k \leq+\infty$ and $\kappa=0$, we set

$$
\begin{gathered}
\widehat{\boldsymbol{\psi}}_{\varepsilon}(x, t):=\overline{\boldsymbol{\psi}}_{\varepsilon}(x, t)+r_{\varepsilon} \boldsymbol{w}_{1 \varepsilon}\left(x, t, \frac{y_{\varepsilon}\left(x_{3}\right)}{r_{\varepsilon}}\right) \\
\boldsymbol{w}_{1 \varepsilon}\left(x, t, y_{3}\right):=\left(\begin{array}{c}
-\frac{\partial \bar{\psi}_{\varepsilon 3}}{\partial x_{1}} y_{3} \\
-\frac{\partial \bar{\psi}_{\varepsilon 3}}{\partial x_{2}} y_{3} \\
-l_{\varepsilon}\left(\frac{\partial \bar{\psi}_{\varepsilon 1}}{\partial x_{1}}+\frac{\partial \bar{\psi}_{\varepsilon 2}}{\partial x_{2}}\right) y_{3}
\end{array}\right) .
\end{gathered}
$$

where the function $y_{\varepsilon}($.$) is defined by (4.9).$

(ii) If $0<\kappa \leq+\infty$, we set

$$
\begin{aligned}
& \widehat{\boldsymbol{\psi}}_{\varepsilon}(x, t):=\overline{\boldsymbol{\psi}}_{\varepsilon}(x, t)+r_{\varepsilon} \boldsymbol{w}_{1 \varepsilon}\left(x, t, \frac{y_{\varepsilon}\left(x_{3}\right)}{r_{\varepsilon}}\right)+r_{\varepsilon}^{2} \boldsymbol{w}_{2 \varepsilon}\left(x, t, \frac{y_{\varepsilon}\left(x_{3}\right)}{r_{\varepsilon}}\right), \\
& \boldsymbol{w}_{1 \varepsilon}\left(x, t, y_{3}\right):=-\frac{\partial \bar{\psi}_{\varepsilon 3}}{\partial x_{1}} y_{3} \boldsymbol{e}_{1}-\frac{\partial \bar{\psi}_{\varepsilon 3}}{\partial x_{2}} y_{3} \boldsymbol{e}_{2} \\
& \boldsymbol{w}_{2 \varepsilon}\left(x, t, y_{3}\right):=\frac{l_{\varepsilon}}{2\left(l_{\varepsilon}+2\right)}\left(\frac{\partial^{2} \bar{\psi}_{\varepsilon 3}}{\partial x_{1}^{2}}+\frac{\partial^{2} \bar{\psi}_{\varepsilon 3}}{\partial x_{2}^{2}}\right) y_{3}^{2} \boldsymbol{e}_{3} .
\end{aligned}
$$

It is usefull to notice that $\widehat{\boldsymbol{\psi}}_{\varepsilon}$ is continuously differentiable in $C_{\varepsilon} \times(0, T)$ (see (6.7)), that $\widehat{\boldsymbol{\psi}}_{\varepsilon}=0$ if $\kappa=+\infty$ (because then, by (6.4), $\boldsymbol{\psi}=0$ ) and that for $m \in\{1,2\}$,

$$
\begin{aligned}
& \left(\left|\widehat{\boldsymbol{\psi}}_{\varepsilon}(x, t)-\boldsymbol{\psi}(x, t)\right|+\left|\frac{\partial^{m} \widehat{\boldsymbol{\psi}}_{\varepsilon}}{\partial t^{m}}(x, t)-\frac{\partial^{m} \boldsymbol{\psi}}{\partial t^{m}}(x, t)\right|\right) \mathbb{1}_{C_{\varepsilon}}(x) \leq C r_{\varepsilon}, \\
& \left|\nabla \widehat{\boldsymbol{\psi}}_{\varepsilon}(x, t)\right| \mathbb{1}_{C_{\varepsilon}} \leq C .
\end{aligned}
$$

Lemma 7.1. Let $\boldsymbol{u}_{\varepsilon}$ be the solution to (3.5). Let $I_{3 \varepsilon}$ be defined by (7.1) in terms of $\widehat{\boldsymbol{\psi}}_{\varepsilon}$ described above. Then,

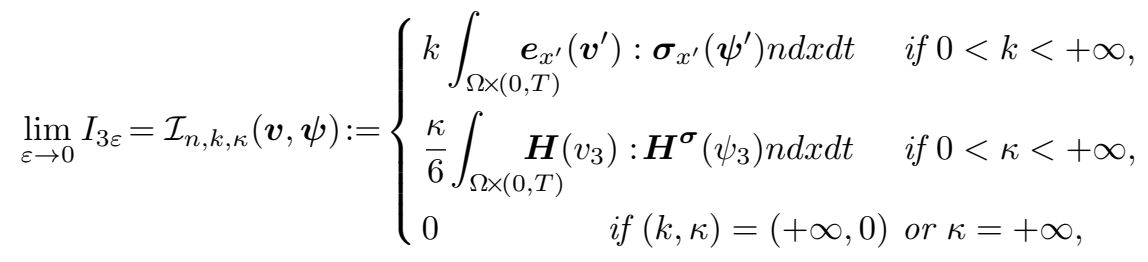

the operators $\boldsymbol{H}, \boldsymbol{H}^{\boldsymbol{\sigma}}$ being defined by 


$$
\begin{aligned}
\boldsymbol{H}(\psi) & :=\left(\begin{array}{ccc}
\frac{\partial^{2} \psi}{\partial x_{1}^{2}} & \frac{\partial^{2} \psi}{\partial x_{1} \partial x_{2}} & 0 \\
\frac{\partial^{2} \psi}{\partial x_{1} \partial x_{2}} & \frac{\partial^{2} \psi}{\partial x_{2}^{2}} & 0 \\
0 & 0 & 0
\end{array}\right) ; \\
\boldsymbol{H}^{\boldsymbol{\sigma}}(\psi) & :=\left(\begin{array}{ccc}
2 \frac{l+1}{l+2} \frac{\partial^{2} \psi}{\partial x_{1}^{2}}+\frac{l}{l+2} \frac{\partial^{2} \psi}{\partial x_{2}^{2}} & \frac{\partial^{2} \psi}{\partial x_{1} \partial x_{2}} & 0 \\
\frac{\partial^{2} \psi}{\partial x_{1} \partial x_{2}} & \frac{l}{l+2} \frac{\partial^{2} \psi}{\partial x_{1}^{2}}+2 \frac{l+1}{l+2} \frac{\partial^{2} \psi}{\partial x_{2}^{2}} & 0 \\
0 & 0 & 0
\end{array}\right) .
\end{aligned}
$$

Proof. Case $0<k<+\infty$. We easily check that

$$
\begin{aligned}
& \left\|\boldsymbol{\psi}-\overline{\boldsymbol{\psi}}_{\varepsilon}\right\|_{L^{\infty}\left(B_{\varepsilon} \times(0, T)\right)}+\left\|\frac{\partial\left(\boldsymbol{\psi}-\overline{\boldsymbol{\psi}}_{\varepsilon}\right)}{\partial x_{\alpha}}\right\|_{L^{\infty}\left(B_{\varepsilon} \times(0, T)\right)} \leq C r_{\varepsilon} \quad(\alpha \in\{1,2\}), \\
& \left\|\frac{\partial^{2}\left(\boldsymbol{\psi}-\overline{\boldsymbol{\psi}}_{\varepsilon}\right)}{\partial x_{\alpha} \partial x_{\beta}}\right\|_{L^{\infty}\left(B_{\varepsilon} \times(0, T)\right)} \leq C r_{\varepsilon} \quad(\alpha, \beta \in\{1,2\}) .
\end{aligned}
$$

A straightforward computation yields (see (3.5), (3.6))

$$
\begin{aligned}
& \boldsymbol{\sigma}_{\varepsilon}\left(\widehat{\boldsymbol{\psi}}_{\varepsilon}\right) \mathbb{1}_{B_{\varepsilon}}= \\
& \mu_{1 \varepsilon}\left(\begin{array}{ccc}
2 \frac{\partial \bar{\psi}_{\varepsilon 1}}{\partial x_{1}}+\frac{2 l_{\varepsilon}}{l_{\varepsilon}+2}\left(\frac{\partial \bar{\psi}_{\varepsilon 1}}{\partial x_{1}}+\frac{\partial \bar{\psi}_{\varepsilon 2}}{\partial x_{2}}\right) & \frac{\partial \bar{\psi}_{\varepsilon 1}}{\partial x_{2}}+\frac{\partial \bar{\psi}_{\varepsilon 2}}{\partial x_{1}} & 0 \\
\frac{\partial \bar{\psi}_{\varepsilon 1}}{\partial x_{2}}+\frac{\partial \bar{\psi}_{\varepsilon 2}}{\partial x_{1}} & 2 \frac{\partial \bar{\psi}_{\varepsilon 2}}{\partial x_{2}}+\frac{2 l_{\varepsilon}}{l_{\varepsilon}+2}\left(\frac{\partial \bar{\psi}_{\varepsilon 1}}{\partial x_{1}}+\frac{\partial \bar{\psi}_{\varepsilon 2}}{\partial x_{2}}\right) & 0 \\
0 & 0 & 0
\end{array}\right) \mathbb{1}_{B_{\varepsilon}} \\
& +r_{\varepsilon} \mu_{1 \varepsilon} O(1) .
\end{aligned}
$$

Since $\frac{r_{\varepsilon}}{\varepsilon} \mu_{1 \varepsilon} \rightarrow k \in(0,+\infty)$ and $l_{\varepsilon} \rightarrow l \in(0, \infty)$ (see (3.6)), we infer from (7.7) that

$$
\lim _{\varepsilon \rightarrow 0}\left|\frac{r_{\varepsilon}}{\varepsilon} \boldsymbol{\sigma}_{\varepsilon}\left(\widehat{\boldsymbol{\psi}}_{\varepsilon}\right)-k \boldsymbol{\sigma}_{x^{\prime}}\left(\boldsymbol{\psi}^{\prime}\right)(x, t)\right|_{L^{\infty}\left(B_{\varepsilon} \times(0, T)\right)}=0,
$$

where $\boldsymbol{\sigma}_{x^{\prime}}\left(\boldsymbol{\psi}^{\prime}\right)$ is given by (2.1). By Corollary 5.6 (i), the convergences (5.11) are verified, thus the sequence $\left(\boldsymbol{e}_{x^{\prime}}\left(\boldsymbol{u}_{\varepsilon}^{\prime}\right) m_{\varepsilon}\right)$ weakly* converges in $L^{\infty}\left(0, T ; \mathcal{M}\left(\bar{\Omega} ; \mathbb{S}^{3}\right)\right)$ to $n \boldsymbol{e}_{x^{\prime}}\left(\boldsymbol{v}^{\prime}\right)$. Taking (3.39), (5.5), and (7.1) into account, we deduce that

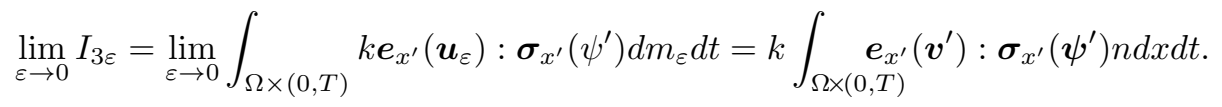

Case $(k, \kappa)=(+\infty, 0)$. By $(6.4),(7.8)$, we have $\left|\boldsymbol{\sigma}_{\varepsilon}\left(\widehat{\boldsymbol{\psi}}_{\varepsilon}\right) \mathbb{1}_{B_{\varepsilon}}\right| \leq C \mu_{1 \varepsilon} r_{\varepsilon}$, thus, by (3.8), the second line of (5.5), and (7.1), there holds

$$
\begin{aligned}
I_{3 \varepsilon} & \leq C \mu_{1 \varepsilon} r_{\varepsilon} \int_{B_{\varepsilon} \times(0, T)}\left|\boldsymbol{e}\left(\boldsymbol{u}_{\varepsilon}\right)\right| d x d t=C \mu_{1 \varepsilon} \frac{r_{\varepsilon}^{2}}{\varepsilon} \int_{B_{\varepsilon} \times(0, T)}\left|\boldsymbol{e}\left(\boldsymbol{u}_{\varepsilon}\right)\right| d m_{\varepsilon} d t \\
& \leq C \mu_{1 \varepsilon} \frac{r_{\varepsilon}^{2}}{\varepsilon} \sqrt{\int_{B_{\varepsilon} \times(0, T)}\left|\boldsymbol{e}\left(\boldsymbol{u}_{\varepsilon}\right)\right|^{2} d m_{\varepsilon} d t} \leq C \mu_{1 \varepsilon} \frac{r_{\varepsilon}^{2}}{\varepsilon} \sqrt{\frac{\varepsilon}{r_{\varepsilon} \mu_{\varepsilon 1}}} \leq C \sqrt{\frac{r_{\varepsilon}^{3}}{\varepsilon} \mu_{1 \varepsilon}}=o(1) .
\end{aligned}
$$


Case $0<\kappa<+\infty$. A straightforward computation gives

$$
\begin{aligned}
& \frac{r_{\varepsilon}^{2}}{\varepsilon} \boldsymbol{\sigma}_{\varepsilon}\left(\widehat{\boldsymbol{\psi}}_{\varepsilon}\right) \mathbb{1}_{B_{\varepsilon}}= \\
&-2 \frac{r_{\varepsilon}^{3}}{\varepsilon} \mu_{1 \varepsilon} \frac{y_{\varepsilon}\left(x_{3}\right)}{r_{\varepsilon}}\left(\begin{array}{rrr}
\frac{2\left(l_{\varepsilon}+1\right)}{l_{\varepsilon}+2} \frac{\partial^{2} \bar{\psi}_{\varepsilon 3}}{\partial x_{1}^{2}}+\frac{l_{\varepsilon}}{l_{\varepsilon}+2} \frac{\partial^{2} \bar{\psi}_{\varepsilon 3}}{\partial x_{2}^{2}} & \frac{\partial^{2} \bar{\psi}_{\varepsilon 3}}{\partial x_{1} \partial x_{2}} & 0 \\
\frac{\partial^{2} \bar{\psi}_{\varepsilon 3}}{\partial x_{1} \partial x_{2}} & \frac{l_{\varepsilon}}{l_{\varepsilon}+2} \frac{\partial^{2} \bar{\psi}_{\varepsilon 3}}{\partial x_{1}^{2}}+\frac{2\left(l_{\varepsilon}+1\right)}{l_{\varepsilon}+2} \frac{\partial^{2} \bar{\psi}_{\varepsilon 3}}{\partial x_{2}^{2}} & 0 \\
0 & 0 & 0
\end{array}\right) \mathbb{1}_{B_{\varepsilon}} \\
&+r_{\varepsilon} O\left(\frac{r_{\varepsilon}^{3}}{\varepsilon} \mu_{\varepsilon}\right) .
\end{aligned}
$$

We deduce from (3.6), (3.8), (7.6), and (7.7), that

$$
\lim _{\varepsilon \rightarrow 0}\left|\frac{r_{\varepsilon}^{2}}{\varepsilon} \boldsymbol{\sigma}_{\varepsilon}\left(\widehat{\boldsymbol{\psi}}_{\varepsilon}\right)+2 \kappa \boldsymbol{H}^{\boldsymbol{\sigma}}\left(\psi_{3}\right) \frac{y_{\varepsilon}\left(x_{3}\right)}{r_{\varepsilon}}\right|_{L^{\infty}\left(B_{\varepsilon} \times(0, T) ; \mathbb{S}^{3}\right)}=0 .
$$

By (3.39) and (7.1), we have

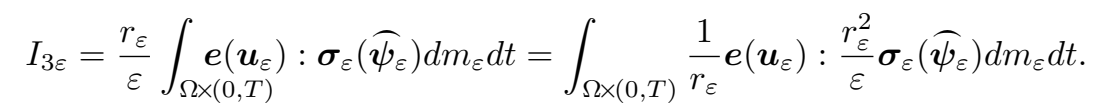

Taking Corollary 5.6 (ii) into account, we infer from (5.5), (5.30), (5.32), (7.9), (7.10), that

$$
\begin{aligned}
& \lim _{\varepsilon \rightarrow 0} I_{3 \varepsilon}=\lim _{\varepsilon \rightarrow 0}-\int_{\Omega \times(0, T)} \frac{1}{r_{\varepsilon}} \boldsymbol{e}_{x^{\prime}}\left(\boldsymbol{u}_{\varepsilon}\right): 2 \kappa \boldsymbol{H}^{\boldsymbol{\sigma}}\left(\psi_{3}\right) \frac{y_{\varepsilon}\left(x_{3}\right)}{r_{\varepsilon}} d m_{\varepsilon} d t \\
& =-2 \kappa \sum_{\alpha, \beta=1}^{2} \int_{\Omega \times(0, T) \times I}\left(\boldsymbol{H}^{\boldsymbol{\sigma}}\left(\psi_{3}\right)\right)_{\alpha \beta}\left(\frac{1}{2}\left(\frac{\partial \xi_{\alpha}}{\partial x_{\beta}}+\frac{\partial \xi_{\beta}}{\partial x_{\alpha}}\right)(x, t)-\frac{\partial^{2} v_{3}}{\partial x_{\alpha} \partial x_{\beta}}(x, t) y_{3}\right) y_{3} n d x d t d y_{3} \\
& =\frac{\kappa}{6} \int_{\Omega \times(0, T)} \boldsymbol{H}\left(v_{3}\right): \boldsymbol{H}^{\boldsymbol{\sigma}}\left(\psi_{3}\right) n d x d t .
\end{aligned}
$$

Acknowledgments. The research of the author is supported by the french National Research Agency (ANR-13-BS03-0009-03).

\section{REFERENCES}

[1] Z. Abdessamad, I. Kostin, G. Panasenko, V. P. Smyshlyaev, Memory effect in homogenization of a viscoelastic Kelvin-Voigt model with time-dependent coefficients. Math. Models Methods Appl. Sci. 19 (2009), no. 9, p.p. 1603-1630.

[2] A. Amosov, I. Kostin, G. Panasenko, V. P. Smyshlyaev, Homogenization of a thermochemo-viscoelastic Kelvin-Voigt model. J. Math. Phys. 54 (2013), no. 8, 081501, 12 pp.

[3] G. Allaire, Homogenization and two-scale convergence, SIAM J. Math. Anal., 23 (1992), pp. $1482-1518$.

[4] Y. Amirat, K. Hamdache, A. Ziani, Etude d'une équation de transport à mémoire, C. R. Acad. Sci. Paris Sér. I Math., 311 (1990), pp. 685-688.

[5] T. Arbogast, J. Douglas, U. Hornung, Derivation of the double porosity model of single phase flow via homogenization theory, SIAM J. Math. Anal., 21 (1990), pp. 823-836. 
[6] N.O. Babych, I.V. Kamotski, V.P. Smyshlyaev, Homogenization in periodic media with doubly high contrasts, Networks and heterogeneous Media, 3 (3) (2008), 413-436.

[7] M. Bellieud, A notion of capacity related to linear elasticity. Applications to homogenization. Arch. Ration. Mech. Anal., vol. 203, no1, (2012), pp. 137-187.

[8] M. Bellieud, Torsion effects in elastic composites with high contrast. SIAM J. Math. Anal. 41(6) (2010), pp. 2514-2553.

[9] M. Bellieud, Problèmes capacitaires en viscoplasticité avec effets de torsion. C. R. Math. Acad. Sci. Paris 351 (2013), no. 5-6, 241-245.

[10] M. Bellieud, Quelques problèmes d'homogénéisation à fort contraste en élasticité. Habilitation à diriger les recherches, Université Montpellier II, (2013).

[11] M. Bellieud, G. Bouchitté, Homogenization of elliptic problems in a fiber reinforced structure. Non local effects. Ann. Scuola Norm. Sup. Cl. Sci. IV 26 (1998), pp. 407-436.

[12] M. Bellieud, G. Bouchitté, Homogenization of a soft elastic material reinforced by fibers, Asymptot. Anal., 32, No. 2 ( 2002), pp. 153-183.

[13] M. Bellieud, I. Gruais, Homogenization of an elastic material reinforced by very stiff or heavy fibers. Non local effects. Memory effects, J. Math. Pures Appl., 84 (2005), pp. $55-96$.

[14] M. Bellieud, C. Licht, G. Michaille, S. Orankitjaroen , Nonlinear capacitary problems for a non periodic distribution of fibers. Appl Math Res Express, (2014) (1), pp. 1-51.

[15] M. Sh. Birman, T. A. Suslina, Averaging of periodic differential operators taking a corrector into account. Approximation of solutions in the Sobolev class $H^{2}\left(\mathbb{R}^{d}\right)$. (Russian) Algebra i Analiz 18 (2006), no. 6, pp. 1-130; translation in St. Petersburg Math. J. 18 (2007), no. 6, pp. $857-955$

[16] M. Sh. Birman, T. A. Suslina, Averaging of periodic elliptic differential operators taking a corrector into account. (Russian) Algebra i Analiz 17 (2005), no. 6, pp. 1-104; translation in St. Petersburg Math. J. 17 (2006), no. 6, pp. 897-973

[17] G. Bouchitté, Représentation intégrale de fonctionnelles convexes sur un espace de mesures. Ann. Univ. Ferrara Sez. VII (N.S.) 33 (1987) pp. 113-156.

[18] G. Bouchitté, C. Picard, Singular perturbations and homogenization in stratified media, Applicable Analysis, Vol. 61 (1996), pp. 307-341.

[19] A. Braides, M. Briane, Homogenization of non-linear variational problems with thin lowconducting layers, Appl. Math. Optim., 55, No. 1 (2007), pp. 1-29.

[20] M. Camar-Eddine, P. Seppecher, Determination of the closure of the set of elasticity functionals, Arch. Ration. Mech. Anal., 170 (2003), pp. 211-245.

[21] K. B. Cherednichenko, Two-scale asymptotics for non-local effects in composites with highly anisotropic fibres, Asymptotic analysis, 49 (2006), pp. 39-59.

[22] K. B. Cherednichenko, M. Cherdantsev, Two-scale $\Gamma$-convergence of integral functionals and its application to homogenisation of nonlinear high-contrast periodic composites. Arch. Ration. Mech. Anal., vol. 204, (2012), pp. 445-478.

[23] K. B. Cherednichenko, V. P. Smyshlyaev, V. V. Zhikov, Non-local homogenised limits for composite media with highly anisotropic periodic fibres, Proceedings of the Royal Society of Edinburgh: Section A 136 (2006), pp. 87-114.

[24] S. Cooper, Homogenisation and spectral convergence of a periodic elastic composite with weakly compressible inclusions, Appl. Anal. 93 (2014), no. 7, pp. 1401-1430.

[25] R. Dautray, J.L. Lions, Analyse mathématique et calcul numérique pour les sciences et les techniques, 8, Evolution: semi-groupe, variationnel, Masson (1988).

[26] G. A. Francfort, F. Murat, Homogenization and optimal bounds in linear elasticity, Arch. Rational Mech. Anal. 94 (1986), no. 4, pp. 307-334.

[27] B. Gustafsson, J. Mossino, Г-convergence of stratified media with measure-valued limits, Asymptot. Anal., 22 (2000), pp. 261-302.

[28] B. Gustafsson, J. Mossino, Compensated compactness for homogenization and reduction of dimension: the case of elastic laminates, Asymptot. Anal., 47 (2006), pp. 139-169.

[29] B. Gustafsson, J. Mossino, C. Picard, H-convergence for stratified structures with high conductivity, Adv. math. sci. appl., 4 (1994), pp. 265-284.

[30] B. Heron, J. Mossino, H-convergence and regular limits for stratified media with low and high conductivity, Applicable Analysis 57 (1995), pp. 271-308., 7, $\mathrm{n}^{0} 1$, (1994), pp. 157-178.

[31] B. Heron, J. Mossino, C. Picard, Homogenization of some quasilinear problems for stratified media with low and high conductivities, Differential and Integral Equations, $7, \mathrm{n}^{0} 1$, (1994), pp. $157-178$.

[32] M. HNID, Étude de transmission à travers des inclusions minces faiblement conductrices de "codimension un": homogénéisation et optimisation des structures, RAIRO, Modélisation mathématique et analyse numérique, 24, $\mathrm{n}^{0} 5$, (1990), pp. 627-650. 
[33] R. James, R. Lipton, A. Lutoborski, Laminar elastic composites with crystallographic symmetry, SIAM J. Appl. Math. 50 (1990), no. 3, pp. 683-702.

[34] E. Y. Khruslov, Homogenized models of composite media, Progress in Nonlinear Differential Equations and their Application, Birkhäuser (1991), pp. 159-182.

[35] J. L. Lions, Equations différentielles opérationnelles et problèmes aux limites, Grundlehren Bd. 111, Berlin / Göttingen / Heidelberg: Springer (1961).

[36] J. L. Lions, E. Magenes, Problèmes aux limites non homogènes et applications, 1, Dunod, Paris (1968).

[37] M. L. Mascarenhas, Memory effects phenomena and Gamma-convergence, Proc. Roy. Soc. Edinburgh, 123 A (1993), pp. 311-322.

[38] W. H. MC CONNEL, On the approximation of elliptic operators with discontinuous coefficients, Ann. Scuola Norm. Sup. Pisa 43 (1) (1976), pp. 123-137.

[39] F. Murat, L. TARTAR, Calcul des variations et homogénéisation, In: Homogenization methods: theory and applications in physics, pp. 319-370. Bréau-sans-Nappe, 1983, Eyrolles, Paris, 1985.

[40] S. Neukamm, Rigorous derivation of a homogenized bending-torsion theory for inextensible rods from 3d elasticity. Arch. Ration. Mech. Anal., vol. 206 (2), (2012), pp. 645-706.

[41] G. NGuetseng, A general convergence result for a functional related to the theory of homogenization, SIAM J. Math. Anal., 20 (1989), pp. 608-623.

[42] O. A. Oleinik, A. S. Shamaev, G. A. Yosifian, Mathematical problems in elasticity and homogenization, Stud. Math. Appl., North Holland (1992).

[43] M. A. Pakhnin, T. A. Suslina, Operator error estimates for the homogenization of the elliptic Dirichlet problem in a bounded domain. (Russian) Algebra i Analiz 24 (2012), no. 6, pp. 139-177; translation in St. Petersburg Math. J. 24 (2013), no. 6, pp. 949-976.

[44] G. P. PANASEnKo, Multicomponent homogenization of processes in strongly nonhomogeneous structures, Sb. Math. vol. 69, no. 1 (1991), pp. 143-153.

[45] G. P. PANASEnKo, Multicomponent homogenization of the vibration problem for incompressible media with heavy and rigid inclusions, C.R. Acad. Sci. Paris, t. 321, Série I, (1995), pp. 1109-1114.

[46] C. Pideri, P. Seppecher, A second gradient material resulting from the homogenization of an heterogeneous linear elastic medium, Contin. Mech. Thermodyn., 9 (1997), pp. 241-257.

[47] G.V. SANDRAKOv, Homogenization of elasticity equations with contrasting coefficients (Russian), Mat. Sbornik, 190 (12) (1999), p.p. 37-92; English translation in Sbornik Math., 190 (12) (1999), p.p. 1749-1806., Zh. Vychisl. Mat. i Mat. Fiz., 44:10 (2004), p.p. 1829-1844; English transl. Comput. Math. Math. Phys., 44:10 (2004), pp. 1741-1756.

[48] G.V. SANDRAKov, Multiphase models of nonstationary diffusion in homogenization, Zh. Vychisl. Mat. i Mat. Fiz., 44:10 (2004), p.p. 1829-1844; English transl. Comput. Math. Math. Phys., 44:10 (2004), pp. 1741-1756.

[49] G.V. SANDRAKOV, Multiphase homogenized diffusion models for problems with several parameters, Izvestiya RAN: Ser. Mat. 71:6 (2007), pp. 119-182, English transl. Izvestiya: Mathematics 71:6 (2007), pp. 1193-1252.

[50] A. Sili, Homogenization of the linearized system of elasticity in anisotropic heterogeneous thin cylinders, Math. Meth. Appl. Sci. vol. 25, Issue 4 (2002), pp. 263-288.

[51] J. Simon, Compact sets in the space $L^{p}(0, T ; B)$ Annali di Matematica pura ed applicata (IV), Vol. CXLVI, pp. 65-96.

[52] L. TARTAR, Remarks on homogenization, Homogenization and Effective Moduli of Materials and Media, J.L. Eriksen, D. Kinderlehrer, R. Kohn and J.L. Lions, eds, Springer, 1986, pp. 228-246.

[53] L. TARTaR, Memory effects and Homogenization, Arch. Rat. Mech. Anal. vol. III, no 2, pp. 121-133, 1990.

[54] L. Tartar, Cours Peccot, Collège de France, (1977), unpublished, partially written in F. MuRAT, L. TARTAR, "H-convergence", in Topics in the Mathematical Modelling of Composite Materials, ed. by L. Cherkaev, R. V. Kohn, Progress in Nonlinear Differential Equations and their Applications, 31, Birkaüser, Boston (1998), pp. 21-43.

[55] V. V. ZHIKOv, Homogenization of elasticity problems on singular structures, Izvestiya: Mathematics, 2002, Volume 66, Number 2, pp. 299-365.

[56] S.E. Pastukhova, V. V. Zhikov, Homogenization for elasticity problems on periodic networks of critical thickness, Sbornik Mathematics, 2003, 194 (5), pp. 697-732. 